The Assessment and Development of Oppositionality 
Paranimfen: Ewout Hoffenaar Rosa van Loon

Printed by Print Partners Ipskamp B.V., Enschede Copyright Peter Hoffenaar, Amsterdam, 2004 All rights reserved 


\section{The Assessment and Development of Oppositionality}

\section{ACADEMISCH PROEFSCHRIFT}

ter verkrijging van de graad van doctor aan

de Vrije Universiteit Amsterdam, op gezag van de rector magnificus prof.dr. T. Sminia,

in het openbaar te verdedigen

ten overstaan van de promotiecommissie van de faculteit der Psychologie en Pedagogiek op vrijdag 17 december 2004 om 10.45 uur in de aula van de universiteit,

De Boelelaan 1105

door

Petrus Johannes Hoffenaar

geboren te Alkmaar 
promotor: prof.dr. J.M. Koot

copromotor: dr. J.B. Hoeksma 
"If you obey all the rules, you miss all the fun" (Katharine Hepburn) 



\section{Contents}

\section{Chapter 1}

Introduction $\quad 9$

\section{Chapter 2}

The structure of oppositionality: Response dispositions and situational aspects

\section{Chapter 3}

Self-reported oppositionality: Individual and situational differences

\section{Chapter 4}

Understanding informant disagreement on children's conduct problems

\section{Chapter 5}

Rebellious teens? :

Examining developmental changes in oppositionality during early adolescence

\section{Chapter 6}

Developmental significance of oppositionality during early adolescence:

Trajectories toward academic, social, and psychological adjustment

\section{Chapter 7}

General discussion

Nederlandse samenvatting

[Summary in Dutch]

\section{References}

\section{Dankwoord}

[Acknowledgments in Dutch] 
CHAPTER 1

\section{Introduction}


Oppositionality consists of a wide range of behaviors in a variety of situations. Temper outbursts, stubborn and negative behavior, disobedience, and hostility have all been noted as behaviors related to oppositionality (Gard \& Berry, 1987). These behaviors are usually observed in relation to those in authority, but can also occur in situations involving other persons with whom the child is familiar, such as peers (Greene \& Doyle, 1999). Behaviors like resistance to adult directions and unwillingness to negotiate with peers have in common that they reflect the child's refusal to conform to social rules. The core element of oppositionality appears to be a willful contrariness; the child does not do what he or she is supposed to be doing (Redl, 1976).

Oppositionality has rarely been studied as a distinct phenomenon (Greene \& Doyle, 1999). It is mostly studied in combination with more serious conduct problems or impulsiveness and hyperactivity. This relative neglect may be partly due to difficulties in the conceptualization of oppositionality. One of the most serious difficulties is how to distinguish incidental instances of noncompliance from the excessive noncompliance and negativity that characterize oppositionality (Wenar, 1982). The categorical view and the dimensional view of oppositionality represent distinct solutions to this problem in the assessment of oppositionality.

The studies reported in this thesis took a dimensional approach to address the assessment and development of oppositionality. First, we addressed the issue of assessment. The first question of the present thesis was whether a new self-report instrument, the Amsterdam Scale of Oppositionality (ASO), could provide researchers and clinicians with a valuable tool for assessing oppositionality. We addressed the validity of this instrument and the problem of situational specificity in the assessment of oppositionality and children's problem behavior in general. Second, we studied the development of oppositionality during early adolescence as well as the outcomes related to interindividual differences in the development of oppositionality. These studies will be introduced in more detail in the following sections of this introduction.

\section{The assessment of oppositionality and conduct problems}

The categorical view of oppositionality relies on the assumption that some children can be diagnosed as oppositional and that these children differ qualitatively from normal children (Hinshaw \& Park, 1999). In a categorical approach clinically relevant levels of oppositionality are diagnosed by means of a classification system in which the disorder is defined by specific criteria. According to the DSM-IV, a recurrent pattern of negativistic, hostile and defiant behavior for at least six months makes a child eligible for the diagnosis Oppositional Deviant Disorder (ODD; American Psychiatric Association, 1994). The categorical view assumes that there is a definable boundary between normal versus pathological expressions of oppositionality. However, until now, few psychological 
syndromes have been identified that conform to this assumption (Ruscio \& Ruscio, 2004), and we are not aware of any study demonstrating this for oppositional behavior.

In contrast, the dimensional view is based on the assumption that oppositionality is a continuum, ranging from normal resistance to a constellation of disruptive actions, including temper tantrums, extreme disobedience and negativistic, hostile behaviors (Gard \& Berry, 1987). The assumption of an underlying continuum implies that the boundaries between normal and abnormal remain either unspecified or vague (Blashfield \& Livesley, 1991). In a dimensional approach to the assessment of psychopathology, psychological tests or rating scales are the proper measurement instruments.

Despite the fact that oppositional behaviors occur throughout the general population (Anthony, 1976; Gard \& Berry, 1987) and proposals for a shift towards a more dimensional model of classification have been recently forwarded (Widiger \& Clark, 2000), researchers and clinicians interested in measuring oppositionality along a continuum of responses have almost no suitable instruments at their disposal. Most of the accepted rating scales or behavior checklists are based on the diagnostic criteria of the DSM-IV category ODD and consist of items describing rather extreme forms of oppositionality. As a result, these instruments seem unfit to measure the full range of variation in oppositionality, including the normative and the pathological. The present dissertation starts by examining the psychometric properties of a recently developed self-report instrument to measure oppositionality in early adolescence along a continuum, the Amsterdam Scale of Oppositionality (ASO). This scale was specifically constructed to measure the full range of oppositionality (Lagendijk, 1997; Leeuwen, 1996; Matten, 1995). Only items portraying everyday oppositional interactions that are bound to occur in every child's life (e.g. refusing to clean his/her own room or going to bed in time) were included.

Chapter 2 details the theoretical notions on which the construction of the ASO was based and reports on a study of its structural validity. In light of the increased attention to the role of emotions in normal and disruptive behaviors (Cole, Michel, \& Teti, 1995; Cole \& Zahn-Waxler, 1992; Dodge, 1991; Eisenberg et al., 1996) and the interpersonal component of oppositionality (Mones, 1998), prior conceptualizations of oppositionality were reconsidered. By comparing models with different factor structures, it was tested whether oppositionality is better conceptualized as a combination of emotional and behavioral responses varying across situations, i.e. interactions with parents, teachers, and peers. The data came from a crosssectional study of a representative sample of children living in the Netherlands $(N=1196)$, aged 8 to 13 years.

Based on the same sample, Chapter 3 continues to investigate whether the ASO is a psychometrically sound instrument by examining its criterion-related validity. Children are in the unique position to report on their behavior across a wide range of situations, some of which are not readily accessible to parents or teachers. Although some studies have cast doubt on the validity of self-reports of oppositionality (Loeber, Green, Lahey, \& Stouthamer- 
Loeber, 1989), there is now ample evidence to support the validity of self-reports in the assessment of children's behavior (Kamphaus, DiStefano, \& Lease, 2003). This chapter focuses on the validity of self-reported oppositionality by examining whether the ASO provides valid information about the patterning of children's oppositional responses across situations. For this purpose, self-reported oppositionality as obtained by means of the ASO was related to parent and teacher ratings on the Strength and Difficulties Questionnaire (SDQ; Goodman, 1997).

Chapter 4 changes the perspective on factors influencing the assessment of problem behaviors by focusing on parent and teacher ratings of children's conduct problems (obtained from the SDQ). The aim of this study is to better understand why parents and teachers disagree on children's conduct problems. The study focuses on variations in the type of conduct problems, variations in the type of situations, as well as variations in the type of observers. Each of these sources of variation has been noted as a factor likely to reduce agreement between informants (van der Ende, 1999).

Traditionally, the assessment of disruptive behaviors relies more heavily on reports of adult informants than on self-reports. However, parents and teachers are likely to have only limited access to the child's full behavioral repertoire, because they usually do not get the chance to observe children outside the context in which they naturally operate (i.e. home and school context). Because children's behavior is likely to vary across situations, situational specificity has been put forward as an explanation for the lack of agreement between informants (Achenbach, McConaughy, \& Howell, 1987). Explaining the lack of agreement from situational differences in the child's behavior implies that each informant provides valid information about the child's behavior in specific situations. Although this assumption seems reasonable, there is little empirical evidence to support it. Burns, Walsh, and Gomez (2003) showed that at least for symptoms of hyperactivity and inattention there is suggestive evidence for the assumption that parents provide unique information about the child's behavior at home and that teachers provide unique information about the child's behavior at school. Using a somewhat different approach, we aim to show that conduct problem ratings of different informants contain valid situation-specific information.

\section{Development of oppositionality}

The two final chapters (5 and 6) of this thesis focus on the development of oppositionality. They present the findings from a longitudinal study aimed to understand the developmental course and longitudinal outcomes of oppositionality in early adolescence. A community sample of 242 boys and 256 girls was assessed in a longitudinal study with six measurement occasions during early adolescence (9-13 years) and half-year intervals between occasions. We used the Amsterdam Scale of Oppositionality (ASO; Hoffenaar \& Hoeksma, 2002) to repeatedly assess oppositionality. At the last measurement occasion, a point in time that coincided with the transition from elementary school to secondary school, a wide variety 
of developmental outcomes was assessed. The variety of outcomes included aspects of the broad domains of academic competence, social competence, and psychological adjustment. They were assessed by using multiple informants.

In chapter 5, we present our research efforts to describe the developmental course of oppositionality during early adolescence using children's self-reported oppositionality assessed on six measurement occasions across the preadolescent to early adolescent period. Basic descriptive data on the developmental course of oppositionality are a prerequisite to understand causes and underlying developmental processes. As Lahey et al. (2000) put it: "One cannot explain what has not yet been described..." (p. 489).

Public opinion holds that early adolescence is a period of heightened "storm and stress". The general public expects young adolescents to be rebellious and sees increases in opposition during early adolescence as typical. Although there is little evidence to justify a representation of early adolescence as a period in which "storm-and-stress" is inevitable and universal, there are at least two important reasons to examine the development of oppositionality during early adolescence. First, influential scholars in the field argue that the "storm-and-stress" view may not be entirely incorrect (Arnett, 1999; Steinberg, 2000). They emphasize individual differences in the experience of early adolescence, but recognize that many young adolescents become more willing to disagree with those in authority and may show an increase in oppositionality. So, although only a part of all young adolescents may experience "storm-and-stress", parents and teachers may rightfully perceive early adolescence as a relatively more difficult time. Second, despite the supposedly special developmental status of early adolescence, no studies have specifically focused on the development of oppositionality during this developmental period. Previous longitudinal studies examined developmental changes in oppositionality across broad age ranges and used relatively large intervals between measurement occasions (Bongers, Koot, van der Ende, \& Verhulst, 2004; Nagin \& Tremblay, 1999). This probably hindered any attempts to estimate change separately across different developmental periods. Early adolescence is widely recognized as a period of developmental transition (Granic, Hollenstein, Dishion, \& Patterson, 2003; Larson, Moneta, Richards, \& Wilson, 2002; Paikoff \& Brooks-Gunn, 1991) in which biological maturation and increasing social cognitive abilities bring about changes in how young adolescents perceive themselves and significant others. Therefore, it seems worthwhile to focus on developmental changes in oppositionality during this specific period.

Chapter 6 puts the observed developmental changes in oppositionality during early adolescence as described in chapter 5 in perspective. This chapter takes a developmental approach, inspired by new methods for testing predictive developmental hypotheses (Hoeksma \& Knol, 2001), to empirically evaluate the significance of oppositionality during early adolescence. This chapter will answer important questions concerning the consequences of oppositionality. Does oppositionality put children at risk for poor academic achievement? To what extent does oppositionality affect children's social functioning? And are oppositional 
children able to express their autonomy within the constraints of interdependent relationships (Kuczynski \& Hildebrandt, 1997) without doing harm to the quality of these interpersonal relationships?

The goal of understanding the outcomes of oppositionality fits nicely with the goal of developmental psychopathology to study adaptive and maladaptive functioning from a developmental perspective. Kazdin, Kraemer, Kessler, Kupfer, and Offord (1997) noted several ways in which the study of antecedent conditions and subsequent outcomes can contribute to the field of developmental psychopathology. Most importantly, this type of research can direct attention to risk-mechanisms and to possible causal relations. Linking developmental trajectories of oppositionality during the last years of elementary school (antecedent condition) to indicators of children's academic, social, and psychological functioning (subsequent outcomes) may make an even greater contribution than more "traditional" risk-factor research, because the developmental process is explicitly taken into account. We aimed to establish not only the impact of different levels of oppositionality on developmental outcomes but also the impact of changes in oppositionality. Information on the link between both the mean level and rate of change of oppositionality and later outcomes is expected to provide more clues and stronger pieces of evidence regarding risk-mechanisms and developmental processes.

Finally, in chapter 7, the results presented in the foregoing chapters will be integrated and discussed. Several common themes that run throughout multiple chapters of this thesis will be identified and discussed. In addition, this chapter will discuss the implications of our results and provide recommendations for future research efforts based on the strengths and limitations of our studies. 


\section{CHAPTER 2}

\section{The Structure of Oppositionality}

Hoffenaar, P. J., \& Hoeksma, J. B. (2002). The structure of oppositionality: Response

dispositions and situational aspects. Journal of Child Psychology and Psychiatry, 43, 375385 . 
Oppositional behavior typically ranges from actions of normal protest when autonomy is threatened to tyrannical aggressive behaviors that seem to satisfy the child's needs (Gard \& Berry, 1987). The child refuses requests, commands or breaks implicit rules. His or her main battle is with not doing what he or she is supposed to do (Redl, 1976). Oppositional behavior demands attention when it starts to frustrate parents and teachers or threatens the welfare of others. Possibly for that reason, the assessment of oppositional behavior largely focuses on psychopathology. The DSM-IV (American Psychiatric Association, 1994) is the main instrument for the clinical assessment of oppositional deviant behavior. A child, showing a recurrent pattern of negativistic, hostile and defiant behavior for at least six months becomes eligible for the diagnosis Oppositional Deviant Disorder (ODD). Other available instruments, such as the Child and Adolescent Psychiatric Assessment (CAPA; (Angold \& Costello, 1996) and The Child and Adolescent Disruptive Behavior Inventory (CADBI; (Burns et al., 1997), are based on the DSM criteria.

Oppositional behaviors are observed at all ages and occur throughout the general population (Anthony, 1976; Gard \& Berry, 1987). In addition, oppositionality is a research topic with a long history (Brehm, 1981). During this longstanding research tradition different subject headings, e.g. resistant behavior, negativistic behavior and noncompliance, have been used to refer to oppositionality in children.

Although oppositionality is a common phenomenon, psychometric instruments to assess the full range of oppositional behaviors are rare. This situation seems to change at the moment. Drabick, Strassberg \& Kees (2001) developed an instrument that specifically targets noncompliance in preschool children. Recently, we developed the Amsterdam Scale of Oppositionality (ASO), to measure oppositionality in early adolescence by means of selfreport. Preliminary versions of the scale were piloted by van der Matten (1995), van Leeuwen (1996) and Lagendijk (1997). The present study is based on the third and final revision of the ASO.

The Amsterdam Scale of Oppositionality (ASO) was constructed according to a facetdesign. Fiske (1971) stressed the importance of the facet-design as a heuristic aid for itemconstruction. The facet approach forces the researcher to explicate his theoretical notions regarding the construct to be measured. The resulting theoretical framework leads to hypotheses and predictions about the role of the facets and the relations among the elements within the facets (Edmundson, Koch, \& Silverman, 1993). Tests of these hypotheses have implications for the structural validity of the instrument. To assure the structural validity of the ASO the internal structure of the instrument should parallel the structure of the oppositionality construct (Loevinger, 1957). 


\section{Theoretical notions}

Three theoretical notions guided the construction of the Amsterdam Scale of Oppositionality. First, oppositionality is conceived of as a continuum. Secondly, the content domain of oppositionality is thought to be represented by both emotions and behaviors across different situations. Finally, oppositional responses are seen as partially situation specific. These notions will be discussed next.

\section{Oppositionality as a continuum}

It is not necessary to take a position in the never-ending controversy on quantitative psychometric instruments versus qualitative psychiatric classification (Blashfield \& Livesley, 1991; Jensen, Brooks-Gunn, \& Graber, 1999) to argue that oppositionality can be seen as a continuum. The continuum of oppositionality ranges from normal resistance to external influences to a constellation of disruptive actions, including temper tantrums, extreme disobedience and negativistic, hostile behaviors (Anthony, 1976; Gard \& Berry, 1987; Redl, 1976). The diagnostic DSM-IV category Oppositional Defiant Disorder (ODD) lies at the abnormal end of the continuum of oppositionality. ODD comprises behaviors closely related to more serious disruptive behaviors as aggression, status violations and property violations. Oppositional behavior is less problematic and non-destructive (Loeber, Lahey, \& Thomas, 1991b).

Rather than starting at the abnormal extreme of the continuum, the ASO was constructed by starting to investigate the full range of oppositional responses in the normal population. The main advantage of basing an instrument on an underlying continuum of responses is that it increases the functionality of the instrument. First, the instrument becomes well suited for research in the normal population, whereas the resulting scale can still be applied for the assessment of deviant child behavior. Deviant behavior is conceptualized as a higher rate and intensity of oppositional actions. It should be noted that this does not imply that children who frequently respond in an oppositional way, should be diagnosed as children with ODD. Second, measuring the full range of oppositionality increases the usefulness of the scale for developmental research. Developmental changes in oppositionality are more easily revealed by instruments that measure the full range of oppositional responses. A categorical approach with instruments that only differentiate between normal- and deviant behavior, seems less suited for developmental research.

\section{Emotions and behavior}

Oppositionality is not necessarily restricted to behavior. Oppositional emotions are another important aspect of oppositionality. Recent literature shows an increased attention for the role of emotions in normal and disruptive behaviors (Cole et al., 1995; Cole \& ZahnWaxler, 1992; Dodge, 1991; Eisenberg et al., 1996). The importance of the role of emotions 
was illustrated in studies by Cole \& Zahn-Waxler (1992) and Cole, Michel \& Teti (1995). Their results provide support for a specific pattern of emotional responding that disruptive children have in common. Disruptive children appear to under-control their anger and display a lack of fear or social anxiety (Eisenberg et al., 1996). Furthermore, these children are less inclined to display sadness and sometimes become overwhelmed by joy when this is not appropriate (Cole \& Zahn-Waxler, 1992).

We assume that oppositional children have a distinct pattern of emotional responding. When they are disciplined, oppositional children possibly express joy, whereas guilt or shame would be a more socially acceptable response. When autonomy is threatened, these children may be more inclined to respond with anger. In addition, they often show a relative lack of fear for disciplinary actions.

In our view oppositionality refers to both behaviors and emotions. This is reflected in the ASO by the fact that one half of the items refer to emotional responses and the other half refer to behavioral responses. As a consequence, the question as to what extent oppositional behavior and emotions are related can be answered.

\section{Situational specificity versus pervasiveness}

Oppositionality occurs in response to directives or prohibitions by different social agents (Brehm, 1981). Most of these social agents (e.g. teachers, parents, and other adults) operate in specific contexts (e.g. home and school). The items of the ASO portray interactions with parents, authority figures, and peers to reflect the different situations in which oppositionality occurs. Adults other than the child's parents are seen as authority figures, but most of the items pertain to teachers.

Different contexts may elicit different responses from children. Some children show oppositional behavior only at home, whereas others display oppositionality in more than one setting (Rey \& Walter, 1999). Because of the situational variance in oppositionality, the judgments of teachers and parents probably do not necessarily correspond. Findings from studies of inter-informant agreement (Achenbach et al., 1987; Fisher \& Fagot, 1996) show that the informant's judgment is context-specific. Achenbach et al. (1987) conclude from their comprehensive meta-analysis that the assessment of children's behavioral and emotional problems is not only affected by informant variables, but also by situational variables. $\mathrm{He}$ argues that the disagreement between informants is at least partly due to situational variance in the child's behavior.

We hypothesize that oppositional emotions and responses are partly situation specific. Items of the ASO pertain to three different situations, namely situations with parents, peers, and authority figures. This variability offers the opportunity to determine the extent to which oppositionality is general over various situations and to what extent it is situation-specific. 


\section{Gender differences}

As far as we know, gender differences with regard to oppositionality have hardly been studied. Nevertheless the literature offers several cues why gender differences are to be expected. Leadbetter, Kuperminc, Blatter \& Hertzog (1999) argue that girls have more difficulties in expressing anger than boys. The difference is attributed to a heightened interpersonal vulnerability of girls. Girls are more concerned about the quality and the maintenance of interpersonal relationships. Because of their sensitivity to interpersonal concerns, girls are possibly more inclined to refrain from behavior that affects relationships negatively.

The same reasoning could hold with respect to the expression of oppositionality. When a child displays oppositionality, this evokes frustration and annoyance in the person whose authority is questioned (Anthony, 1976). Assuming that girls are more inclined to refrain from behaviors with negative interpersonal consequences, girls will try to withhold their oppositionality. For that reason, we hypothesize that girls in general display less oppositional emotions and oppositional behavior than boys.

A second cue from the literature suggests that gender differences in oppositionality may be even more pronounced in certain situations. Kavanagh and Hops (1994) discuss several research findings, suggesting that parents and teachers are likely to have more consistent expectations and reinforce behavior more congruously for girls than for boys. Parents are more indulgent and tolerate more excessive behavior from boys than teachers do. At school, boys will generally meet a more restrictive social environment.

Because boys are used to a wide range of acceptable behaviors at home, they will experience the more severe restrictions at school as elimination of perceived behavioral freedoms. The elimination of an established freedom may lead to oppositionality (Brehm, 1981). For that reason, we expect boys to display oppositionality more frequently and more intensely in interactions within the school context.

In sum, we theorize that oppositionality consists of interrelated oppositional emotions and oppositional behaviors. Oppositional emotions and oppositional behaviors are hypothesized to be partially situation specific. Oppositionality occurs in interactions with parents, authority figures, and peers. We expect gender differences in the level of oppositional behavior and the level of oppositionality across contexts. 


\section{Method}

\section{Participants}

Participants were children (age 8-12 years) from 20 Dutch primary schools. A nationwide self-weighting cluster sample was drawn from a register of all Dutch primary schools. Probability of inclusion for each school was inversely proportional to the mean school size per province (data on school size were provided by the Dutch Central Bureau for Statistics, CBS, 1997). Schools were contacted by phone. Approximately one in two schools refused participation for reasons not related to the variables of interest. The main reasons given were: "We are too busy" and "We already participated in research some time ago". When a school refused participation, the next school in the sample register replaced this school. Within schools, complete classes were examined. The number of refusals within classes was less than $1 \%$.

The initial sample contained 1,196 children. It was reduced by the deletion of $3.18 \%$ of the children, because of more than $20 \%$ of incomplete data per child. In the reduced sample, the average percentage of missing values amounted to $1.67 \%$. Missing values for the remaining children were imputed using corrected item mean substitution (CIMS; Huisman, 1999 ). CIMS replaces missing values by the item mean corrected for the individual's total score on the observed items. Bernaards \& Sijtsma (2000) recently demonstrated in a simulation study that imputation methods based on an individual's mean score yield the best results in recovering the factor loading structure from the incomplete data.

The final sample contained 1,158 children, 560 boys and 598 girls. The mean age was $11.41 \mathrm{yr}(S D=.93)$.

\section{Amsterdam Scale for Oppositionality}

The Amsterdam Scale for Oppositionality (ASO) contains 30 forced-choice items. Analysis of the reliability of the ASO showed that the internal consistency $(\alpha=.86)$ was adequate.

As noted earlier, the ASO was constructed according to a facet-design (Fiske, 1971). The content domain of oppositionality is defined by a response facet and a situational facet. The elements of the response facet are oppositional emotions and oppositional behaviors. Three elements make up the situational facet: interactions with authority figures, peers, and parents. Each item represents either an emotional response or a behavioral response in one of the three situations. For every item the child chooses between an oppositional and a nonoppositional response alternative.

The ASO contains five items for each combination of the response- and situational facet elements. Table 1 illustrates the item content, for each combination of the situational facet and response facet. 
Table 1.

Item content by situation and response type.

\begin{tabular}{|c|c|c|}
\hline & \multicolumn{2}{|c|}{ Response } \\
\hline Situation & Behavior & Emotion \\
\hline Authority & $\begin{array}{l}\text { When the teacher says I have to quit talking, } \\
\text { I keep my mouth shut/I keep on talking }\end{array}$ & $\begin{array}{l}\text { When I'm punished by the teacher, } \\
\text { I feel sad/I feel angry }\end{array}$ \\
\hline Peers & $\begin{array}{l}\text { Other children think that, } \\
\text { I like to fight/I don't like to fight }\end{array}$ & $\begin{array}{l}\text { When other children steal things, } \\
\text { I don't mind/I think it's awful }\end{array}$ \\
\hline Parents & $\begin{array}{l}\text { When my mum or dad tells me to help them, } \\
\text { I do it/ I pretend I did not hear them }\end{array}$ & $\begin{array}{l}\text { When my mum or dad thinks I'm being rude, } \\
\text { it makes me feel bad/ I think it's funny }\end{array}$ \\
\hline
\end{tabular}

\section{Procedure}

A research assistant or the first author visited the 20 schools. The ASO and a Dutch translation of the Strength and Difficulties Questionnaire (SDQ, Goodman, 1997) were group administered in the classrooms of the 6th, 7th and 8th grades. After a short introduction the questionnaires were distributed and completed individually by all children. Most children concluded the tests in less than twenty minutes. The SDQ is not used in the present study.

\section{Analysis}

The facet structure of the ASO was investigated by means of confirmatory factor analysis (CFA) using EQS (Bentler, 1995). Following the example of Joormann and Stöber (1997), the analysis was conducted using so-called item parcels or subscales. According to the authors, confirmatory factor analytic models using item parcels provide better estimates of the overall fit in comparison to item-based models. Models based on item parcels are estimated more precisely, because of the higher reliability of parcels (relative to individual items) and the smaller number of parameters.

Item parcels were created by randomly dividing the five items for every combination of the response- and situational facet elements into subgroups of two or three items. Next, item parcels were adjusted for the unequal number of items by dividing the score by the number of items.

According to the main hypothesis of the present study, oppositionality consists of interrelated oppositional emotions and oppositional behaviors and is partially situation specific. This hypothesis can be represented by a five-factor model, as shown in Figure 1. 
Figure 1.

Simplified representation of the five-factor model for the structure of oppositionality.

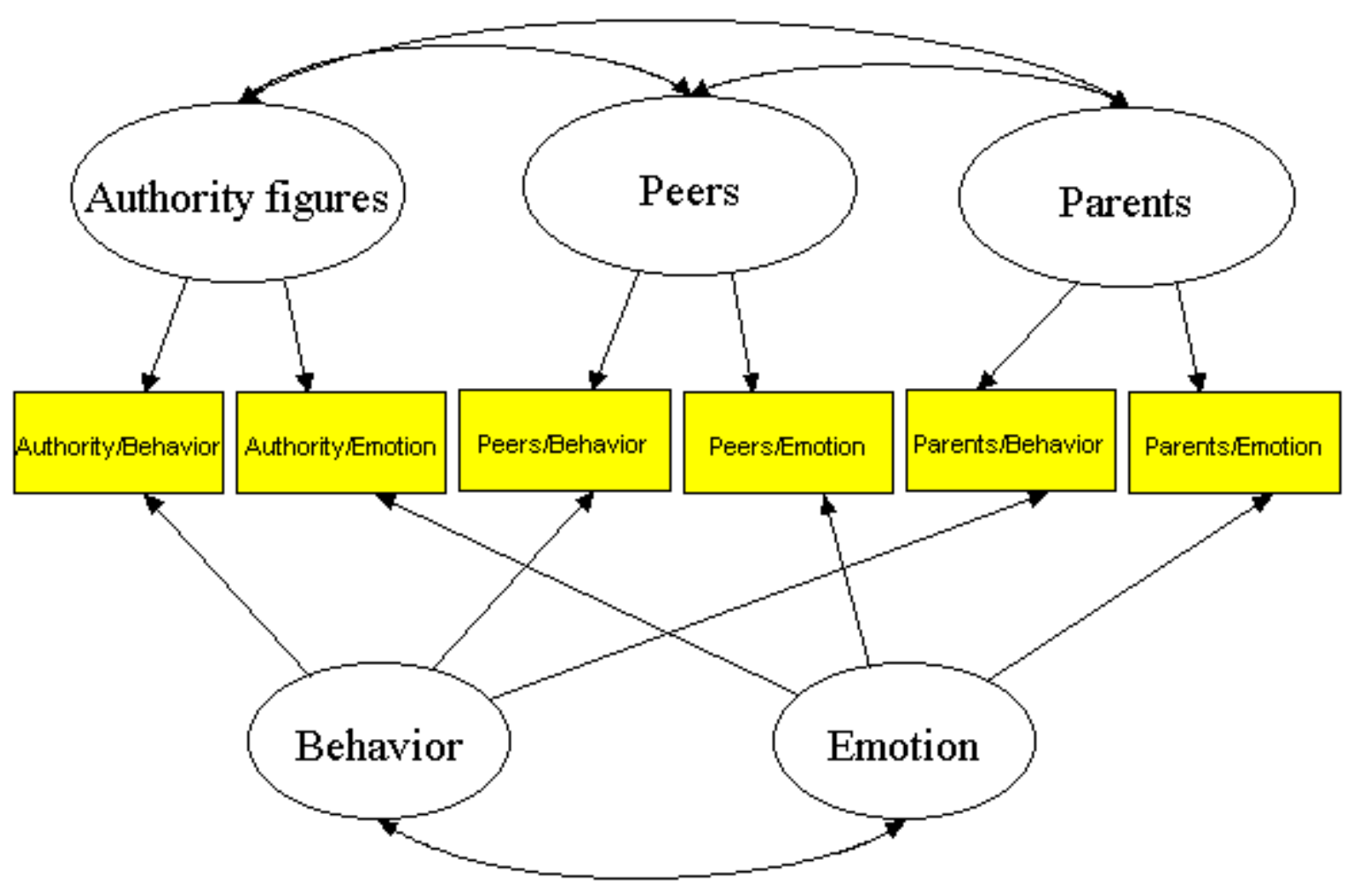

In Figure 1, each factor represents one facet element. Each item parcel loads on two factors, one factor representing the corresponding response facet element and one factor representing the corresponding situational facet element.

The structure of the ASO was investigated by comparing models with different factor structures in a modeling procedure. The adequacy of the models was evaluated by comparing the theoretical covariance structure as predicted by the model to the observed sample covariance matrix.

The modeling procedure started with testing whether one general oppositional factor underlies all item parcels. The analysis proceeded by testing a model with the two response facet elements as distinct, but related factors. In the next step a model consisting of the three situational facet elements as unrelated factors was tested. In the subsequent analysis a model was tested, consisting of the three situational facet elements as correlated factors. The final model in the procedure was the hypothesized facet structure, as depicted in Figure 1.

\section{Model fit}

The goodness of fit for the various models was assessed by means of the Comparitive Fit Index (CFI, Bentler, 1990 ), Akaike Information Criterion (AIC, Bollen, 1989 ) and the chi-square test. The chi-square test is a likelihood ratio test that evaluates whether the 
restrictions imposed by the model are valid (Bollen, 1989). To compare the fit of the various models, we examined the difference in the fit measures. The likelihood ratio test for the difference in chi-square estimators is also known as the chi-square difference test. The chisquare difference test only allows the direct comparison of nested models. The AIC also provides means of comparing models that are not nested. Akaike Information Criterion is a relative index; the model with the lowest value for the AIC is the preferred model.

In addition to the aforementioned fit indices, the Root Mean Square Error of Approximation (RMSEA; Steiger, 1990) and the Goodness of Fit Index (GFI; Tanaka \& Huba, 1985) are reported for the best fitting model. The following ranges are generally taken to indicate good fit: CFI (.90-1.00); RMSEA (0.03-0.07); GFI (.90-1.00).

\section{Results}

Preliminary analyses revealed that the factor models to be tested were not invariant across gender. The models were therefore evaluated for boys and girls separately. A single factor model was tested first. In the model all item parcels loaded on one general factor. The model embodies the assumption that oppositional emotions and oppositional behaviors cannot be distinguished and do not vary across situations. Test statistics and fit indices (Table 2) showed that the single factor model had to be rejected for both boys and girls.

Table 2.

Model-comparison for boys and girls

\begin{tabular}{lrrrrrrr} 
Model/Test & \multicolumn{2}{c}{ AIC } & \multicolumn{2}{c}{$\chi^{2}$} & df & \multicolumn{2}{c}{ CFI } \\
\hline & \multicolumn{1}{c}{ Boys } & \multicolumn{1}{c}{ Girls } & \multicolumn{1}{c}{ Boys } & \multicolumn{1}{c}{ Girls } & & Boys & Girls \\
One general oppositional factor & 230.90 & 221.58 & 338.90 & 329.58 & 54 & .83 & .85 \\
Two response factors & 225.54 & 223.31 & 331.54 & 329.31 & 53 & .84 & .85 \\
Three situational factors & 448.18 & 463.58 & 556.17 & 571.58 & 54 & .70 & .72 \\
Correlated situational factors & 52.65 & 20.38 & 154.65 & 122.38 & 51 & .94 & .96 \\
Five factor model & -21.91 & 11.81 & 54.10 & 87.82 & 38 & .99 & .97
\end{tabular}

Note. $\mathrm{CFI}=$ Comparative Fit Index, $\mathrm{AIC}=$ Akaike Information Criterion.

The next model fitted contained two factors, referring to the two response facet elements. Item parcels referring to emotions loaded on the first factor and item parcels referring to behaviors loaded on the second factor. The factors were allowed to correlate. The model assumes that oppositional feelings and oppositional behaviors are distinct, but related oppositional responses. Chi-square test statistics and goodness of fit indices showed that the two-factor model had to be rejected. For girls the two factor model did not fit significantly 
better than the single factor model $\left(\chi^{2}(1)=.27, p>0.05\right)$, whereas for boys it $\operatorname{did}\left(\chi^{2}(1)=7.36\right.$, $p<0.01)$.

Subsequently, a three-factor model was fitted. One factor represented oppositional responses in interactions with authority figures. Another factor represented oppositional responses in situations with peers and the final factor involved oppositional responses at home. The model is used to test the assumption that oppositionality is fully situation-specific. For that reason the situational factors were not allowed to correlate. Chi-square test statistics and CFI-values (Table 2) showed that the three-factor model had to be rejected for both boys and girls. The figures in table 2 further reveal that the three-factor model fits very poorly relative to the other models, suggesting that the three situational factors cannot be considered independent.

In the next model the three situational factors were allowed to correlate. This threefactor model embodies the assumption that oppositional responses show some consistency across situations. Compared to the previous three factor model, the fit improved considerably by taking up correlations between the situational factors. Chi-square difference tests showed significant differences in model fit with the previous model for both boys and girls (boys, $\chi^{2}$ $(3)=401.52, p<0.01$; girls, $\left.\chi^{2}(3)=449.20, p<0.01\right)$. The Comparative Fit Index (CFI) appeared to be in the acceptable range and very similar for boys and girls (.94 versus .96). However, the likelihood statistic appeared to be relatively large, indicating the possibility of further improvement of the model.

The final model tested contained five factors. Two factors related to emotional and behavioral responses. The three remaining factors referred to the three different situations (authority figures, peers and parents). The emotional and behavioral response factors were allowed to correlate. The correlations between the situational factors were also included. The model corresponds to the main hypothesis of the present study. According to the hypothesis, oppositionality consists of related oppositional emotions and oppositional behaviors and is partially context-specific. The fit of the five-factor model showed considerable improvement as compared to the three-factor model (boys, $\chi^{2}(13)=100.55, p<0.01$, girls, $\chi^{2}(13)=34.56$, $p<0.01)$. For both boys and girls, the five-factor model showed excellent fit, as indicated by the CFI-values (Table 1). The corresponding Root Mean Square Error of Approximation (RMSEA) and the Goodness of Fit Index (GFI) amounted to respectively RMSEA $=0.051$ and GFI $=.97$ for boys and RMSEA=0.054 and GFI=.97 for girls. All indices pointed to a good fit. For boys the chi-square statistic amounted to $54.10(\mathrm{df}=38, p>0.01)$, indicating acceptable fit. For girls however the chi-square amounted to 87.82 ( $\mathrm{df}=38, p<0.01)$, suggesting the model should be rejected. Given the large sample size and the fact that all other fit-indices pointed to good fit, the five-factor model for girls was taken as the best fitting model.

In sum the analyses lead to the acceptance of the five-factor model, strongly supporting the notion that oppositionality consists of interrelated emotions and behaviors and that oppositionality is partially situation specific. 
Questions pertaining to the relation between oppositional emotions and oppositional behavior and to the degree of situational specificity of oppositionality remain. Table 3 displays the correlations between the factors in the five-factor model for boys and girls. The correlation between the response factors reflects the relation between oppositional feelings and oppositional behavior. The correlations between the situational factors reflect the consistency of oppositionality across situations.

Table 3.

Estimated correlations between the factors in the five-factor model for boys and girls

\begin{tabular}{lll} 
Correlations & Boys & Girls \\
\hline Response facet elements & & \\
$\quad$ Emotion/Behavior & $.84^{*}$ & $.81^{*}$ \\
Situational facet elements & & \\
Authority/Peers & $.90^{*}$ & $.79^{*}$ \\
Authority/Parents & .25 & $.58^{*}$ \\
Peers/Parents & $.47^{*}$ & $.55^{*}$ \\
\hline
\end{tabular}

Note. ${ }^{*} p<0.05$

Inspection of the correlations between the situational factors (Table 3) showed that for both boys and girls the factors referring to oppositional interactions with authority figures and oppositional interactions with peers were strongly related. The correlations between the situational factors referring to interactions with parents and interactions with authority figures or peers were moderate. For boys the factors referring to oppositional interactions with authority figures and to oppositional responses at home were not significantly related. The results indicate that oppositionality generalizes across situations. Oppositionality in interactions with peers and authority figures appear to have much in common.

The high correlation between the two response factors (Table 3) points to a strong relationship between oppositional emotions and oppositional behavior. It suggests that the response factors may not be distinct. In a separate analysis we tested whether the two response factors should be replaced by a single factor. Replacing the response factors by one general factor in the model for boys resulted in a significant deterioration in model fit $\left(\chi^{2}(1)=\right.$ 11.34, $p<0.01)$. In the model for girls, the fit did not deteriorate $\left(\chi^{2}(1)=1.4, p>0.05\right)$. However, the parameter estimates did not appear to be interpretable. For that reason the distinctiveness of the two response factors was also accepted for girls. In sum, the two response factors are strongly related, but each response factor appears to account for a distinct part of the covariation between item parcels. 


\section{Factor loadings}

Before focusing on the factor loadings, the equivalence of the factor structure across gender was tested. The invariance of the five-factor model across gender was examined using a multi-group procedure. According to this procedure, all factor loadings are first constrained to be equal for boys and girls. Next, the constraints on the factor loadings are released. The likelihood ratio test for the difference between the two models appeared to be significant $\left(\chi^{2}(24)=46.14, p<0.01\right)$, indicating that the factor loadings differed for boys and girls.

Table 4 displays the standardized factor-loadings for the five-factor models for boys and girls. The factor loadings in the model for boys ranged from .10 to .70. In the model for girls they ranged from .00 to .82 . The factor loadings showed a similar pattern in the models for boys and girls. In general, the loadings on the situational factors appeared to be higher and more reliable than the factor loadings on the response factors. These results suggest that a considerable part of the variance is explained by the correlated situational factors.

Table 4.

The five-factor model: standardized factor-loadings and variance extracted estimates.

\begin{tabular}{|c|c|c|c|c|c|c|c|c|c|c|c|c|}
\hline \multirow[t]{2}{*}{$\mathrm{No}^{\mathrm{a}}$. } & \multicolumn{2}{|c|}{$\underline{\text { Behavior }}$} & \multicolumn{2}{|c|}{ Emotion } & \multicolumn{2}{|c|}{ Authority } & \multicolumn{2}{|c|}{ Peers } & \multicolumn{2}{|c|}{ Parents } & \multicolumn{2}{|c|}{ Uniqueness } \\
\hline & Boys & Girls & Boys & Girls & Boys & Girls & Boys & Girls & Boys & Girls & Boys & Girls \\
\hline 1 & $.39 *$ & .22 & & & $.52 *$ & $.61 *$ & & & & & .76 & .76 \\
\hline 2 & $.45^{*}$ & $.28 *$ & & & $.48^{*}$ & $.64 *$ & & & & & .75 & .71 \\
\hline 3 & & & $.64^{*}$ & $.45^{*}$ & $.27 *$ & $.57 *$ & & & & & .72 & .69 \\
\hline 4 & & & $.67 *$ & $.47^{*}$ & $.35^{*}$ & $.54 *$ & & & & & .66 & .70 \\
\hline 5 & $.39 *$ & $.27 *$ & & & & & $.43 *$ & $.52 *$ & & & .81 & .81 \\
\hline 6 & .10 & $.24^{*}$ & & & & & $.64^{*}$ & $.45^{*}$ & & & .76 & .86 \\
\hline 7 & & & $.31^{*}$ & $.23^{*}$ & & & $.49 *$ & $.57 *$ & & & .82 & .79 \\
\hline 8 & & & .17 & .00 & & & $.70 *$ & $.82 *$ & & & .70 & .58 \\
\hline 9 & $.24 *$ & $.17^{*}$ & & & & & & & $.54^{*}$ & $.37 *$ & .81 & .91 \\
\hline 10 & $.48 *$ & $.39 *$ & & & & & & & $.35^{*}$ & $.44^{*}$ & .80 & .81 \\
\hline 11 & & & $.45^{*}$ & .13 & & & & & $.45^{*}$ & $.68^{*}$ & .77 & .72 \\
\hline 12 & & & $.56^{*}$ & $.39 *$ & & & & & $.54^{*}$ & $.68^{*}$ & .63 & .62 \\
\hline V & 5.8 & 3.3 & 14.6 & 6.7 & 5.9 & 13.0 & 11.0 & 10.5 & 7.1 & 10.8 & 55.6 & 55.7 \\
\hline
\end{tabular}

Note. ${ }^{a}$ Number of item parcel. The meaning of the item parcels is found by looking at the presence of factor-loadings. For instance parcels 1 and 2 contain items referring to behavior in situations with authority figures, et cetera. (Consult Table 1 for illustrative items); ${ }^{*}=p<.05, \mathrm{~V}=$ percentage variance explained.

To further examine the relative contribution of the situational factors and response factors, the percentage variance explained by each factor was calculated. The explained variance by a factor corresponds to the sum of the products of the squared factor loadings and variances of the item parcels. The resulting variance component estimates were converted to 
percentages. The final row of Table 4 displays the estimates of the percentage of variance associated with each of the situational factors, the response factors and uniqueness. In the model for boys, the response factors accounted for approximately $20 \%$ of the variance, the situational factors accounted for $24 \%$ of the variance and $56 \%$ of the total variance was due to unreliability of measurement and unique variance associated with each item parcel (residual variance). In the model for girls the response factors explained $10 \%$ of the variance and the situational factors $34 \%$. The remaining $56 \%$ of the variance was residual variance. The estimates of the variance components indicate that for girls the situational factors accounted for three quarters of the variance, whereas for boys the response factors and situational factors explained an approximately equal proportion of the variance.

\section{Gender differences}

Assuming that girls are more inclined to refrain from behaviors with negative interpersonal consequences, we expected girls to display less oppositional behavior than boys. We further hypothesized that gender differences may be more pronounced in the schoolcontext, because for boys the behavior of authority figures is assumed to be inconsistent across contexts.

Table 5.

Means and standard deviations for boys and girls divided by situation and response.

\begin{tabular}{lcrrr}
\hline & \multicolumn{2}{c}{ Boys } & \multicolumn{2}{c}{ Girls } \\
\cline { 2 - 5 } Situation/Response & Emotion & Behavior & \multicolumn{1}{c}{ Emotion } & \multicolumn{1}{c}{ Behavior } \\
\hline Authority & $1.91(1.49)$ & $1.02(1.06)$ & $1.45(1.46)$ & $.87(1.02)$ \\
Peers & $1.39(1.34)$ & $1.34(1.37)$ & $.82(1.19)$ & $.82(1.09)$ \\
Parents & $2.84(2.81)$ & $1.33(1.21)$ & $2.87(2.84)$ & $1.20(1.14)$ \\
& & & & \\
\hline
\end{tabular}

Table 4 displays the means and standard deviations for boys and girls divided by situation and response. Gender differences were analyzed by specifying a three-way repeated measures MANOVA (Response X Situation X Gender) with the response factors and the different situational factors as within subject factors and gender as a between subject factor. The repeated measure analysis revealed a significant Response X Situation X Gender effect $(\mathrm{F}(2,1156)=11.73, p<0.01)$. There appeared to be differences between the mean scores on the emotional responses and behavioral responses. These differences varied across contexts and gender. 
Figure 2.

Boys' and girls' emotional and behavioral responses across situations.

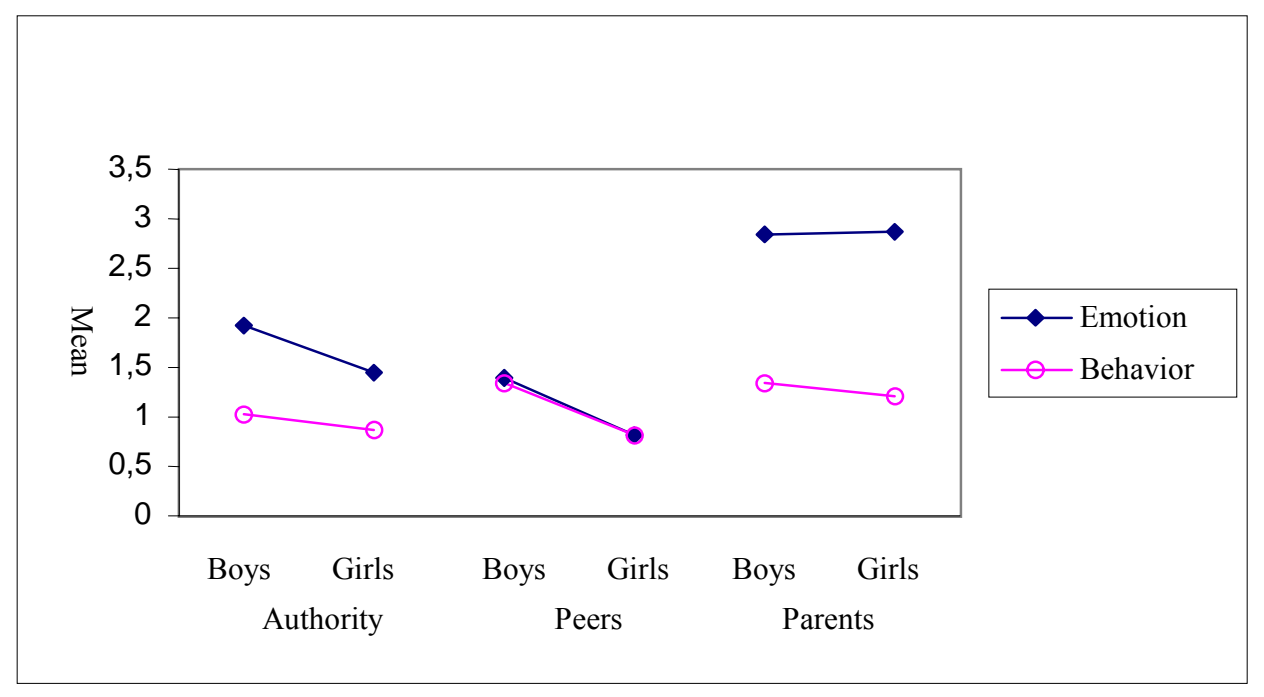

Figure 2 clearly shows how the difference between oppositional emotions and oppositional behavior varies across situations. The mean level of oppositional emotions and oppositional behavior differed most in situations with parents, whereas in situations with peers there appeared to be no difference between emotional or behavioral responses. Figure 2 further shows that gender differences in oppositionality are most pronounced in situations with peers and authority figures (school-context).

The situational dependency of the difference in oppositional emotions and oppositional behavior provided further support for the existence of two separate response categories and underscores the importance of the social context in the display of oppositionality.

\section{Discussion}

This study examined the internal structure of the ASO. The ASO is based on the hypothesis that oppositional emotions and oppositional behaviors, as well as different situations (authority figures, parents, and peers) best represent the content domain of oppositionality. It was hypothesized that oppositionality is partly situation specific. Results of the modeling procedure presented confirm the hypothesis. The poor fit of the one factor model clearly showed that oppositionality cannot be considered a single uni-dimensional construct. The two and three factor models were rejected too, showing that oppositionality cannot be conceived of as a combination of emotional and behavioral dispositions, or as fully determined by specific situations. The final model, corresponding to the main hypothesis, contained three correlated situational factors and two highly correlated general response factors. It provided an adequate representation of the internal structure of the ASO for both boys and girls. In sum the results of the modeling procedure lead to the conclusion that 
oppositionality is best conceptualized as a combination of emotional and behavioral dispositions and mutually related situational effects.

The validity of the difference between emotional and behavioral responses on the one hand and between specific situations on the other is further substantiated by the observed difference in the mean levels of emotional and behavioral responding. This difference varied across situations, providing further support for differentiating behavior, emotions and situations.

Our conclusion regarding the structure of oppositionality raises the question to what extent oppositionality should be attributed to general emotional and behavioral dispositions and to situational effects? Our findings demonstrate that a substantial part of the child's opposition is determined by the specific situation. The variance explained by the three situational factors appeared to be considerable. For boys, situational characteristics accounted for half of the explained variance. For girls, these accounted for three-quarters of the explained variance. Thus, girls are even more affected by the situation than boys. Situational differences were also apparent in the level of emotional responding. The results revealed that children are more likely to show their oppositional feelings to their parents than to teachers and other adults. They are less likely to display oppositional feelings in situations with peers. Note that the level of oppositional behavior was fairly consistent across situations.

Although oppositionality is to a considerable extent determined by the situation, it should be noted that some situations have more in common than others. The correlations between the situational factors showed that oppositionality with peers and oppositionality towards authority figures are strongly related to each other, but only moderately related to oppositionality at home and vice versa.

The observed cross-situational pattern of oppositionality is clearly consistent with the observations by Rey \& Walter (1999), who argued that oppositionality is often restricted to interactions within the family and that "it is less common to find children who are oppositional at school but not at home" (p. 111).

Partial situational specificity was expected. Nevertheless the observed pattern with respect to the correlations and mean levels was somewhat surprising. Why do children express more oppositional feelings to their parents than to teachers and with peers? And what do the latter two situations have in common? The observed pattern probably has to be attributed to differences and commonalties of the consequences of oppositional emotions and behaviors in the different situations. More specifically, we conjecture that the observed pattern of oppositionality is largely due to the child's varying expectations regarding the social or interpersonal consequences of showing oppositionality. Notably Fuchs and Thelen(1988), demonstrated that the child's expectation about (negative) social consequences affects the expression of emotions.

The likelihood of negative consequences, including punishment, retribution and alienation from a close relationship, depends on the specific characteristics of the relationship 
involved. Negative interpersonal consequences are less likely to occur in situations with parents. In general terms the parent-child relationship can be typified as affectionate, secure, non-voluntary and enduring (Bigelow, Tesson, \& Lewko, 1996). The robustness of the parentchild relationship offers children the opportunity to express their feelings (Bigelow et al., 1996), including oppositional feelings.

For different reasons, negative interpersonal consequences are more likely to be expected in situations with authority figures and peers. The child's relationship with teachers is less affectionate, non-voluntary and only partly enduring. It is constrained by formal and social rules (Leman \& Duveen, 1999). Breaking these rules has direct negative interpersonal consequences, i.e. punishment by the teacher and possibly rejection by classmates. A vignette study by Braine, Pomerantz, Lorber \& Kranz (1992) showed that children comply with teachers to avoid punishment.

Relationships with peers can be typified as affectionate, voluntary, and not necessarily enduring. The affectionate and voluntary character of peer relationships strengthens the need to conform (Bigelow et al., 1996; Damon, 1988) and makes the control of feelings especially important (Bigelow et al., 1996). Children are strongly concerned with appearing cool and emotionally in control, especially in middle childhood (Parker \& Gottman, 1989; Underwood, Shockner, \& Hurley, 2001). Thus oppositional feelings may have negative consequences, including alienation from and rejection by peers.

In sum, we maintain that the observed pattern of oppositionality is due to characteristics of the interpersonal relationships that guide expectations about negative consequences of oppositionality in different situations. Future research could be directed at the validity of this proposal.

The topic of situational specificity has also been addressed in the seminal study by Achenbach et al. (1987) on inter-observer agreement regarding the assessment of behavior problems. The authors concluded that the lack of convergence between informants in the assessment of children's behavioral and emotional problems is due to situational variables as well as informant variables. In the design of the studies reviewed, situational effects and informant effects are inevitably confounded. Parents observe children at home and teachers observe children at school. The present study relies on the information of the same 'observers' across different situations, i.e. the children. Our findings point to the importance of the situation. They suggest that the lack of inter-observer agreement between parents and teachers reported by Achenbach et al. (1987) should be attributed to real differences in the child's behavior across different situations and to a lesser extent to differences between informants.

The importance ascribed to the situation should not distract us from the fact that for boys and girls, respectively about one half and one quarter of the explained variance is accounted for by the emotional and behavioral response factors. The factors refer to the child's general tendency or disposition to react with oppositional feelings and oppositional behaviors. The factors are distinct but highly related. The relative values of the variance 
extracted estimates (they were higher for emotions than behavior) point to the fact that emotions are at least as important as behaviors. Several researchers (Greene \& Doyle, 1999; Stifter, Spinrad, \& Braungart-Rieker, 1999) have stressed the role of emotions before. Requests to comply elicit emotional arousal, in particular anger and frustration. The content of the items making up the emotion factor suggest that oppositional emotions are rather diverse. Oppositional children not only respond with anger and frustration, they also display less fear of punishment, sometimes express joy when disciplined and seem less prone to experience shame and guilt when breaking social rules.

Oppositional emotions and oppositional behavior appeared to be strongly related. Children who display more oppositional emotions will also display higher levels of oppositional behavior. According to Frijda's (1986) emotion theory, emotions inherently invoke changes in action readiness. When we adopt this view, the strong relation between emotional and behavioral responses suggests that oppositional behavior is to a large extent driven by emotions. Frustration and anger (in response to requests and prohibitions), and a lack of shame or guilt (for disciplinary actions) all substantially increase the readiness to engage in oppositional behavior.

We hypothesized that girls are more likely to refrain from oppositional behavior than boys, especially within the school context. The hypothesis was based on the assumption of heightened interpersonal vulnerability of girls (Leadbeater et al., 1999) and the presumed strictness of the school environment for boys (Kavanagh \& Hops, 1994). Data analyses revealed a statistically significant interaction between gender, situation and response. Gender differences are, however, most pronounced in situations with peers, less pronounced at school, and virtually absent at home. The findings are only consistent with the supposed interpersonal vulnerability of girls, but do not support the assumption that the strictness of the school environment would lead to high level of oppositionality for boys.

An unexpected gender difference turned up during the modeling procedure. The structure of the models for boys and girls were identical, but factor loadings differed. For girls the situational factors accounted for more variance than the emotion and behavior factors. For boys these proportions were more balanced. The findings suggest that girls are more sensitive to the situation and less predisposed to oppositional responding in general, whereas boys have a stronger general disposition to respond with opposition and are less sensitive to situational characteristics.

In sum, the findings confirm the hypothesis that the oppositionality is best represented as a combination of situational effects and a general tendency to respond with oppositional emotions and oppositional behavior. The findings underscore the role of emotions in oppositionality and the importance of the interpersonal component of oppositionality (Mones, 1998). The findings and conclusions of the present study connect to studies of the role of behavioral and emotional control in the development of oppositionality (Greene \& Doyle, 1999; Stifter et al., 1999), and to research questions pertaining to the situational aspects of 
oppositionality. For both these research questions the ASO provides a valuable addition to the currently available research instruments, because scores can be obtained for oppositional emotions, oppositional behavior and oppositionality in specific situations.

The ASO was constructed on the assumption that oppositionality can be conceived as a continuum. The validity of this assumption cannot be tested directly. At least, all findings together do not contradict the assumption of an underlying continuum.

Although the ASO lacks a clear clinical perspective, it could be useful in the assessment of Oppositional Defiant Disorder. The DSM-IV mentions many emotion-based symptoms for the diagnosis of ODD, e.g. low frustration tolerance and a lack of appropriate feelings of guilt and remorse (American Psychiatric Association, 1994). This recognition of the emotional features of ODD could be continued by using psychometric instruments that provide insight into oppositional emotions.

In the clinical assessment of disruptive behavior disorders, little attention has been paid to the situational aspects of children's behavior (Matthys, Maassen, Cuperus, \& van Engeland, 2001). The frequency and duration of symptoms are recognized as important features for the diagnosis of ODD and other behavioral disorders. The findings of the present study suggest that the situation in which the symptoms occur has been somewhat undeservedly left out of the diagnostic process. 


\section{CHAPTER 3}

\section{Self-reported oppositionality: \\ Individual and situational differences}

Hoffenaar, P. J., Hoeksma, J. B., \& Koot, J.M. (submitted). Self-reported oppositionality: Individual and situational differences. Journal of Clinical Child and Adolescent Psychology 
Oppositional behaviors occur in relationships (Mones, 1998). Anthony (1976) already stated that: “... it takes two to produce oppositional behavior...”(p. 3). As a consequence, the assessment of oppositionality demands consideration of the influences of parents, teachers, and peers (Alexander \& Pugh, 1996). Although the interpersonal component of oppositionality has been recognized for a long time, most current clinical and research models do not explicitly consider differences in oppositional behavior across various relationships. Oppositionality is generally conceptualized as a global child characteristic or trait that is stable across a range of situations. However, by solely relying on the assessment of generalized behavioral tendencies, clinicians and researchers may fail to obtain important information about the patterning of children's oppositional responses across situations (c.f. Wright \& Zakriski, 2001, 2003).

The current study focuses on self-reported oppositionality across different situations. Self-reports offer two important advantages. First, self-reports can provide unique and valuable information about children's own subjective experiences. Child self-reports possess evidence of criterion-related validity (Friman et al., 2000; Kamphaus et al., 2003) and predictive validity (Morgan \& Cauce, 1999). Second, children are in the unique position to report on their behavior across a wide range of situations, some of which are not readily accessible to parents or teachers. Because children are able to report on their oppositional feelings and behaviors in situations with parents, teachers and peers, we can directly compare children's oppositionality across these situations and establish situational differences.

Children's oppositional responses across different situations were obtained by using the Amsterdam Scale of Oppositionality (ASO; Hoffenaar \& Hoeksma, 2002). The ASO is a self-report instrument for children between the ages of 8 and 13 years that aims to measure oppositional emotions and behaviors in multiple situations. For that reason, the format of the ASO is different from that of most other self-report scales. Instead of a list of problem behaviors or symptoms of which children rate the frequency and intensity, the ASO presents children with responses to specific situations and asks them to choose the most symptomatic response alternative. The situations correspond to everyday oppositional interactions in relationships with parents, teachers, and peers.

This study builds on a previous report on the structural validity of the ASO, in which we found that the best fitting factor model matched well with the a priori facet-theoretic design of the instrument (Hoffenaar \& Hoeksma, 2002). It was concluded that oppositionality is best conceptualized as a combination of emotional and behavioral dispositions and mutually related situational effects, and that oppositionality is to a large extent situationspecific. In the present study, we investigated to what extent self-reported oppositionality as obtained by the ASO reflects valid information. In addition, we examined whether children's responses across situations reflect valid information about situational differences in oppositionality. These issues were examined by determining the relation between selfreported oppositionality and criterion behaviors known to be related to oppositionality. 
Conceptually and empirically, oppositionality has been related to various forms of psychopathology and a lack of social adjustment (Loeber, Burke, Lahey, Winters, \& Zera, 2000). Therefore parent and teacher ratings of children's conduct problems, hyperactivity, emotional problems and prosocial behavior were obtained and included as criterion variables.

\section{Criterion variables}

Oppositionality is conceived of as a continuum ranging from normal resistance and mild disobedience to a constellation of disruptive actions, including temper tantrums and negativistic, hostile behaviors (Gard \& Berry, 1987; Hoffenaar \& Hoeksma, 2002). Only behaviors at the far end of this continuum can be found in the DSM-IV criteria for the diagnosis Oppositional Defiant Disorder (ODD; APA, 1994 \#3]). Because there is very little literature available that considers the full range of oppositionality, findings from research on the diagnostic category of ODD may give important clues that could help to formulate expectations with regard to the relations between oppositionality and criterion behaviors.

ODD falls under the heading of disruptive behavior disorders, a category that also includes Attention Deficit Hyperactivity Disorder (ADHD) and Conduct Disorder (CD; APA, 1994). Lack of impulse control is a central feature of the disruptive behavior disorders (Hendren, 1999). Symptoms of oppositionality are often accompanied by symptoms of conduct problems and hyperactivity. The association between oppositionality and other disruptive behaviors has been documented by numerous studies showing a high level of comorbidity of ODD with conduct disorder (CD) and attention hyperactivity disorder (ADHD) (Burns et al., 1997; Hinshaw \& Anderson, 1997; Nottelmann \& Jensen, 1995; Rey \& Walter, 1999). Because oppositionality is tightly linked to conduct problems and hyperactivity, we included these disruptive behaviors as criterion variables. If children provide valid information on their oppositionality, one would expect a positive relation between selfreported oppositionality and adult reports of children's conduct problems and hyperactivity.

Symptoms of oppositionality and emotional problems may also co-occur. Some studies have found an increased risk for depression and anxiety in children with ODD (Greene et al., 2002; Wenning, Nathan, \& King, 1993). In addition, since the correlates of ODD and CD may be closely similar (Rowe, Maughan, Pickles, Costello, \& Angold, 2002), a relation between oppositionality and emotional problems can also be assumed on the basis of the wellestablished comorbidity between CD and emotional problems (Lahey, Loeber, Burke, Rathouz, \& McBurnett, 2002; Loeber et al., 2000). Thus, both oppositionality and conduct problems may be associated with elevated levels of depression and anxiety. The emotional problems of the oppositional child may be the result of feelings of worthlessness and failure, fed by rejection by the peer group or academic failure which are both common in oppositional children (Ledingham, 1999). If self-reports of oppositionality are valid, we would expect a positive relation between oppositionality and emotional problems. 
Oppositionality involves a constant struggle for power and autonomy and as such seems to contrast with prosocial behavior that secures and promotes harmonious relationships. Prosocial behavior refers to positive adjustment to social rules and norms and children's production of positive actions as sharing, helping, and feeling sympathy. Oppositional behavior is characterized by an aversion to social rules and prohibitions made by teachers and parents. Hastings et al. (2000) showed that disruptive children show less concern for others. Similarly, Drabick, Strassberg, and Kees (2001) showed that teacher reports of children's noncompliance were negatively related to prosocial behavior. Oppositionality may also imply a lack of concern for others and in particular for those who make and enforce the rules. If children's self-reports provide valid information about oppositionality, one would expect a negative relation between self-reported oppositionality and adult observations of prosocial behavior. In sum, the validity of self-reported oppositionality as obtained by the ASO will be supported if oppositionality is positively related to both other disruptive behaviors (conduct problems and hyperactivity) and emotional problems, and negatively to prosocial behavior.

\section{Situational differences in oppositionality}

Establishing meaningful relations between self-reported oppositionality and adult ratings of children's conduct problems, hyperactivity, emotional problems and prosocial behavior would indicate that the ASO provides valid information on children's generalized oppositionality, but the ASO may also provide valid information on situational differences in oppositionality. As indicated above, children are in the unique position of being able to report on their feelings and behaviors in multiple situations. In the present study, we examined whether the ASO reflects valid situational differences by relating oppositionality at home, at school and with peers to criterion variables.

Similar to fluctuations in the severity of ADHD symptoms as a result of situational factors (Barkley, 2003), the severity of oppositionality may differ across situations. Characteristics of the situation (home and school) and the relationships involved (with parents, teachers, and peers) are assumed to affect the severity of oppositionality. Families, schools, and peer groups are characterized by different grades of elasticity or flexibility (Henker \& Whalen, 1999). Parents apply rules in a rather flexible way and adapt their behavior to their child's needs, whereas teachers are bound to the rules and constraints of the school. Consequently, teachers have far less possibilities to adapt to individual differences within the classroom. In addition, the school context offers children a well-defined socialorganizational system with a unique emphasis on both performance and social rules (Henker \& Whalen, 1999). Within the school system, teachers hold an official authority position (Laupa, Turiel, \& Cowan, 1995) and classrooms constitute a peer audience.

Because oppositional children react to threats to their (perceived) behavioral freedoms (Brehm, 1981) and their reactions may be strengthened by peer influences and institutional authority, it appears that the school context poses a very demanding environment to 
oppositional children. Assuming that oppositional responses are most strongly triggered at school, individual differences in oppositionality are most likely to be revealed in this situation. Therefore we expect that self-reported oppositionality at school provides more valid information than oppositionality at home or with peers. If the ASO reflects valid situational differences in oppositionality, we would therefore expect stronger relations between oppositionality and criterion behaviors for the school situation, i.e. in oppositional interactions with teachers.

In addition, we examined the validity of situational differences by focusing on the relations between, on the one hand, self-reported oppositionality at home, at school, and with peers, and on the other hand, the reports of two of the recipients of oppositional behaviors, i.e. parents and teachers. Parents and teachers are likely to have only limited knowledge of children's oppositionality outside the context in which they naturally observe children. As a consequence, parents and teachers are likely to provide different information about the child's functioning in different situations (home and school). If we assume that children's oppositionality partially varies across the home and the school context and that parents and teachers mainly observe the child's behavior in these specific situations, parent and teachers may not only have different views about the child's oppositionality, but also about behaviors related to it. If, for example, a child is more oppositional at home than at school, teachers may not only have limited awareness of the child's oppositional behavior, but they may also be less aware about the general repercussions of this behavior (Angold \& Costello, 1996).

The situational specificity of oppositionality and the fact that parents and teachers naturally observe children in different settings lead us to expect unique relations of selfreported oppositionality at home and at school with parent and teacher ratings of criterion variables. If self-reports provide valid situation-specific information, we would expect selfreported oppositionality at home to be more strongly related to parent ratings of criterion behaviors than to teacher ratings. In addition, we would expect oppositionality at school to be more strongly related to teacher ratings than to parent ratings of criterion behaviors.

In sum, we expected that children are not only able to provide valid information on their oppositionality generalized across different situations, but that they are also able to provide valid situation-specific information. If they are able to validly report situational differences, we would expect stronger relations between oppositionality at school and criterion variables. Furthermore, given the situational specificity of adult reports, we would expect that oppositionality at home and at school are differentially related to parent and teacher ratings of criterion behaviors.

\section{Gender differences}

In addition to the primary focus on the validity of self-reported oppositionality in multiple situations, the current study also seized the opportunity to examine the unexplored issue of gender differences in the relations between oppositionality and associated behaviors. 
Recent findings by Moffitt et al. (2001) suggest that patterns of comorbidity among antisocial disorders are highly similar for boys and girls, but ODD was not included in their study. Because most studies have not focused specifically on oppositionality or have only considered boys, the literature offers no further clues regarding potential gender differences in the relation between oppositionality and associated behaviors. Therefore, the present study will explore gender differences in the relation between oppositionality and criterion behaviors.

\section{Method}

\section{Child and Adult Participants}

To obtain a representative sample of Dutch pupils, a nation-wide self-weighting cluster sample of 20 schools was drawn from a register of all Dutch primary schools. Probability of inclusion for each school was inversely proportional to the mean school size per province (data on school size were provided by the Dutch Central Bureau for Statistics, CBS, 1997). Schools were contacted by phone. Approximately one in two schools refused participation for reasons not related to the variables of interest. The main reasons given were: "We are too busy" and "We already participated in research some time ago". When a school refused participation, the next school in the sample register replaced this school. Within schools, children in the 6th, 7th, and 8th grade classes were examined. Parents and caregivers of these children were informed about the research project and could refuse participation by informing the teacher. The number of refusals within classes was always less than $8 \%$.

The initial sample contained 1,194 children. Participating children received a freepost pre-addressed envelope containing a questionnaire with the instruction to give this envelope to their parents. In an appended letter, parents were requested to return the questionnaire. Of the 1,194 parents, $32.5 \%$ did not return the questionnaire. Teachers received questionnaires for each child in their class and a freepost pre-addressed envelope to send completed forms back to the investigator. Of the 52 teachers, 15 did not return the questionnaires. The majority of the other teachers completed the questionnaires for all children in their class, but some teachers failed to do so for a small number of children. In total, $34.1 \%$ of the questionnaires for the teachers were not returned.

Cases were included in this study if complete records were available. Cases with missing information from parents $(22.6 \%)$, teachers $(24.2 \%)$ or both $(9.9 \%)$ were consequently deleted. The sample was further reduced by the deletion of $2.3 \%$ of the cases, because of missing self-report data or other key variables (Gender and Age). The final sample thus consisted of 505 children with complete records. The mean age of the 251 boys was 11.30 years $(S D=.94)$ and the mean age of the 254 girls was 11.32 years $(S D=.85)$. The nonresponse by parents and teachers raises questions about whether the final sample adequately represents the study population. Therefore, the effect of nonresponse will be examined. 


\section{Amsterdam Scale for Oppositionality (ASO)}

The ASO was constructed according to a facet-design (Fiske, 1971). The items were constructed by systematically sampling from the content domain of oppositionality. The content domain of oppositionality was defined by a response facet and a situational facet. The elements of the response facet are oppositional emotions and oppositional behaviors. The elements of the situational facet are situations with parents, teachers, and peers. The items of the ASO represent combinations of the response facet elements and the situational facet elements. Each combination is represented by five forced-choice items, resulting in a total of 30 items. Each item consists of a description of the situation (interactions with parents, teachers or peers), followed by two response alternatives: one oppositional alternative and one non-oppositional response alternative. These alternatives were either emotional responses or behavioral responses.

An example of an item reflecting a behavioral response in interactions with parents is (with assigned scores between brackets): "When my dad or mum tells me to help", "I do it" (0)/ "I pretend I haven't heard" (1). An item reflecting oppositional feelings towards the teacher is: "When the teacher punishes me", "I feel sad" (0)/ "I get angry" (1). An item reflecting an emotional response in situations with peers: "When another child is looking for trouble", "I feel like fighting" (1)/ "I want to walk away" (0).

A previous study examined the structural validity of the ASO using confirmatory factor analyses (Hoffenaar \& Hoeksma, 2002). Supportive evidence was found for a structural model consisting of interrelated emotional and behavioral response factors and three mutually related situational factors. The factors representing the response tendencies and the factors representing the situational effects appeared to be independent. Because oppositionality appeared to be a combination of two independent elements, aggregation over responses within situations is justified.

A generalized oppositionality score is obtained by summing the responses to all items. This score ranges from 0 to 30. In this study, the Cronbach's alpha for generalized oppositionality was $.86(95 \%$ C.I. $=.85-.88)$. Test-retest stability for a sample of children who were tested for a second time after a two-week interval was .89 ( $\mathrm{N}=150)$. Scores for oppositionality in specific situations are obtained by summing the responses within that specific situation. This results in scores for oppositionality at home, at school and in situations with peers that each range from 0 to 10 . In this study, the Cronbach's alphas for oppositionality at home, at school and with peers were $.70(95 \%$ C.I. $=.67-.72), .78(95 \%$ C.I. $=.76-.80)$ and $.75(95 \%$ C.I. $=.73-.77)$, respectively.

\section{Criterion variables: The Strength and Difficulties Questionnaire (SDQ)}

Children's conduct problems, hyperactivity, emotional problems and prosocial behavior were assessed with the corresponding subscales of the Strength and Difficulties 
Questionnaire (SDQ, Goodman, 1997). Each subscale contains five items. Parents, teachers, and children themselves rated the same items on a three-point scale $(0=$ not true, $1=$ somewhat true, $2=$ certainly true). Subscale scores are computed by summing scores on the relevant items. In addition to the scores for parent rated criterion variables and teacher rated criterion variables, we computed a mean adult rating score for the criterion variables by averaging the parent and teacher ratings of a particular criterion variable. To examine the effect of nonresponse, we computed the same subscale scores for the self-report version of the SDQ.

The psychometric properties of the SDQ were recently investigated in a large representative sample (Goodman, 2001). Factor analysis of the SDQ revealed that the items of the SDQ loaded primarily on the predicted factors. In addition, the emotional problems scale and the two disruptive behavior scales appeared to be only moderately related. Support for the predicted factor structure of the SDQ was also found in a large sample of Dutch children (Muris, Meesters, \& van den Berg, 2003). In addition, both studies indicated that the internal consistency, test-retest stability, and inter-informant agreement of the SDQ scales were acceptable.

The concurrent validity of the SDQ was also examined. In the study by Muris, Meesters, and van den Berg, the parent version of the SDQ appeared to be related in theoretically meaningful ways to scores obtained with other measures, including the CBCL, CDI, RCMAS, and ADHDQ. In the study by Goodman, the subscales of the SDQ were found to predict the relevant DSM-IV diagnoses with high specificity. Taken together, these findings demonstrate the utility of the SDQ scales as indices of psychopathology and children's social adjustment.

In the present sample, the Cronbach's alphas for the conduct problems scale were .73 $(95 \%$ C.I. $=.69-.76)$ for the mean adult rating, $.56(95 \%$ C.I. $=.50-.62)$ for the parent ratings, and $.73(95 \%$ C.I. $=.70-.77)$ for the teacher ratings. For hyperactivity, the alpha was $.87(95 \%$ C.I. $=.86-.89)$ for the mean adult rating and the alphas were $.80(95 \%$ C.I. $=.77-.83)$ and .87 $(95 \%$ C.I. $=.85-.89)$ for the parent and teacher ratings, respectively. Alphas for the emotional problems scale were $.72(95 \%$ C.I. $=.69-.76)$ for the mean adult rating, $.67(95 \%$ C.I. $=.62-$ $.71)$ for the parent ratings, and $.77(95 \%$ C.I. $=.74-.80)$ for the teacher ratings. Alphas for the prosocial behavior scale were .75 (95\% C.I. $=.73-.79)$, $.67(95 \%$ C.I. $=.62-.71)$, and $.84(95 \%$ C.I. $=.82-.87)$ for the mean adult rating, parent ratings and teacher ratings, respectively. The alphas for the self-report version of the SDQ were $.47(95 \%$ C.I. $=.42-.51)$, .66 (95\% C.I. $=$ $.63-.69)$, .61 (95\% C.I. $=.57-.64)$, and $.58(95 \%$ C.I. $=.54-.62)$ for conduct problems, hyperactivity, emotional problems, and prosocial behavior, respectively.

\section{Procedure}

A research assistant or the first author visited the 20 schools. The ASO and the selfreport version of the SDQ were group administered in the classrooms of the 6th, 7th, and 8th grades. After a short introduction the scale was distributed and completed individually by all 
children. Most children completed the scales in less than thirty minutes. Teachers were asked to fill out the SDQ for all children in their class. Because of the brevity of the SDQ, we estimate that filling out the questionnaire takes about four minutes per child. There were on average 23 children in a class, which implied about 1,5 hours work for each teacher.

\section{Results}

\section{Effect of nonresponse}

To examine the effect of nonresponse, we focused on possible bias in self-reported oppositionality and the relations between oppositionality and self-reported criterion behaviors as obtained by the self-report version of the SDQ. We compared the 505 participants included in the final sample and the 689 participants who were left out due to missing data or nonresponse by parents and teachers. The mean generalized oppositionality score was 6.82 $(\mathrm{SD}=5.43)$ for the children in the final sample and $7.82(\mathrm{SD}=5.61)$ for the nonresponse group. Although those who were not included in the final sample had significantly higher oppositionality scores, the difference between the two groups of participants accounted for less than $1 \%$ of the variance. Because the actual amount of bias depends on the nonresponse rate and the effect size of the difference, the extent to which the observed difference introduced bias can be calculated more precisely by applying the formula presented by Gerrits, van den Oord, and Voogt (2001). The bias was .006, indicating that the difference between the "observed" mean in the final sample of participants and the true "unobserved" mean in the total sample is negligible.

The correlations of oppositionality with the self-report SDQ scales of hyperactivity $(r=.36)$ and prosocial behavior $(r=-.35)$ were of equal magnitude in the two groups of participants. Oppositionality was not significantly related to emotional problems in both groups $(\mathrm{r}=.00$ and -.04). The correlation between self-reported oppositionality and selfreported conduct problems was somewhat stronger in the group of children with complete information $(\mathrm{r}=.54)$ than in the group of children who were left out due to nonresponse from parents and teachers $(\mathrm{r}=.48)$, but the difference between these two correlation coefficients was not significant. In sum, participants with complete data and those with incomplete data did not appear to substantially differ with regard to their mean level of oppositionality and nonresponse did not appear to have any influence on the correlations between oppositionality and self-reported criterion behaviors as measured by the SDQ. These results suggest that nonresponse did not result in bias in the present study.

\section{Generalized oppositionality}

First, we examined the relations between generalized self-reported oppositionality and the mean adult rating of conduct problems, hyperactivity, emotional problems and prosocial behavior (the average of parent and teacher ratings). In addition, it was tested whether the 
strength of the relations differed for boys and girls. Using SPSS, four regression analyses were conducted with generalized oppositionality as the dependent variable and each of the four criterion variables as independent variables. Gender differences were tested by adding gender and the interaction between gender and the criterion variable to the regression equation. Gender was scored 0 for boys and 1 for girls.

Table 1.

Summary of the regression of oppositionality on conduct problems, hyperactivity, emotional problems, and prosocial behavior obtained from multiple regression analyses

\begin{tabular}{lrrrrr}
\hline Predictor variables & $B$ & $S E B$ & $\beta$ & $t$ & $R$ \\
\hline Intercept & & & & & \\
Conduct Problems & 4.61 & .31 & & $14.92^{* *}$ & \\
& 1.69 & .16 & .41 & $10.20^{* *}$ & .41 \\
Intercept & & & & & \\
Hyperactivity & .25 & .55 & & $7.76^{* *}$ & \\
Gender & .95 & .12 & .42 & $7.78^{* *}$ & .35 \\
Gender*Hyperactivity & .73 & .72 & .07 & 1.00 & .36 \\
& -.54 & .20 & -.19 & $-2.75^{* *}$ & .38 \\
Intercept & 16.86 & 1.18 & & & \\
Prosocial behavior & -1.24 & .14 & -.36 & $-8.67^{* *}$ & .36 \\
Intercept & & & & & \\
Emotional problems & 6.52 & .52 & & $12.65^{* *}$ & \\
Gender & .69 & .22 & .20 & $3.09^{* *}$ & .08 \\
Gender*Emotional problems & -.52 & .74 & -.05 & -.71 & .19 \\
& -.73 & .31 & -.20 & $-2.33^{* *}$ & .21 \\
\hline Note: & & & & & \\
\hline
\end{tabular}

Note: $* p<.05, * * p<.01$

Table 1 summarizes the results of the four regression analyses. Oppositionality was positively related to conduct problems $(\mathrm{r}=.41)$ for boys and girls. The regression analysis revealed no significant gender difference.

The regression analysis for hyperactivity did reveal a significant gender difference. Oppositionality appeared to be strongly positively related to hyperactivity for boys $(\beta=.42)$ and the significant parameter of the gender interaction $(\beta=-.19)$ indicates that the relation between oppositionality and hyperactivity was somewhat less strong for girls. The final regression model for the relation between oppositionality and hyperactivity resulted in a multiple $R$ of .38 .

A significant gender difference was also found in the regression analysis for emotional problems. Oppositionality appeared to be positively related to emotional problems for boys $(\beta=.20)$, but the significant parameter of the gender interaction $(\beta=-.20)$ revealed that oppositionality is not related to emotional problems for girls. The final regression model for the relation between oppositionality and emotional problems resulted in a multiple $R$ of .21 . 
Finally, a regression analyses was conducted with generalized oppositionality as the dependent variable and prosocial behavior as the independent variable. Oppositionality appeared to be negatively related to prosocial behavior $(\mathrm{r}=-.36)$ for boys and girls. The regression analysis revealed no gender difference.

Figure 1.

Regression lines representing the relations between oppositionality and criterion variables for boys and girls.

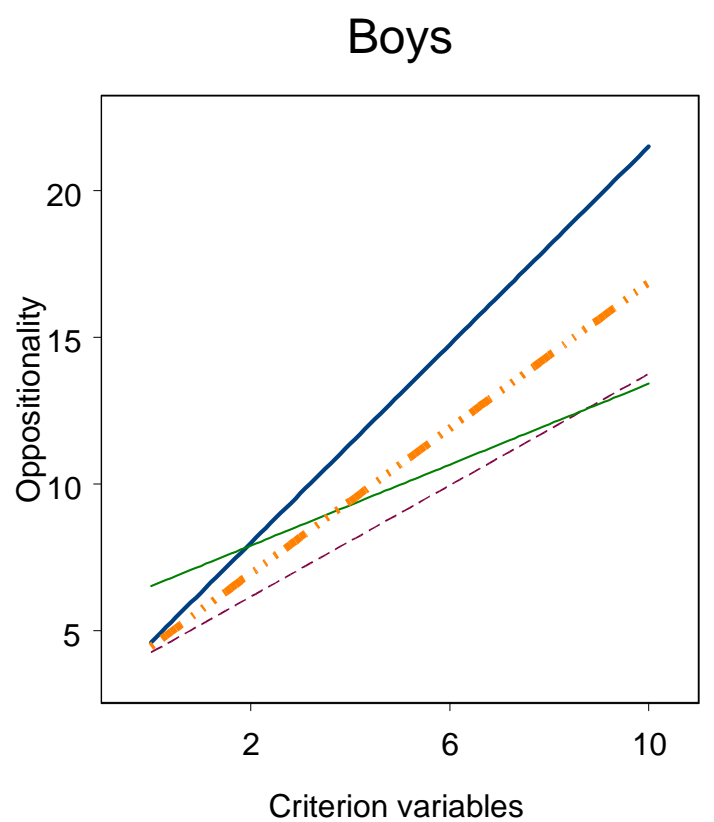

Criterion variables

\section{Girls}

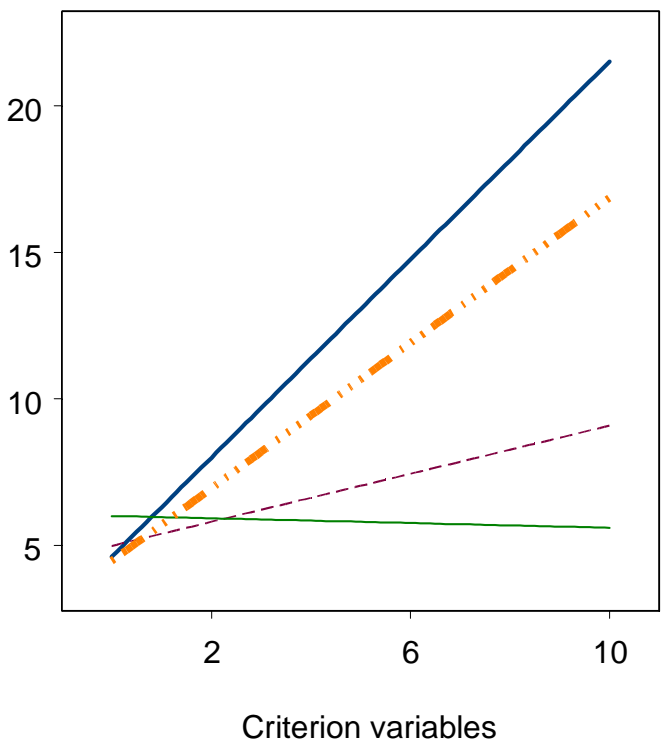

Results are summarized in Figure 1, which graphically displays the regression lines corresponding to the final regression models. To allow a straightforward comparison of the regression lines, prosocial behavior was scaled inversely. Figure 1 shows that self-reported oppositionality is most strongly related to the combined parent and teacher ratings of conduct problems, followed by prosocial behavior and hyperactivity. Comparison of the regression lines for boys and girls again makes clear that oppositionality is more strongly related to hyperactivity for boys than for girls and that oppositionality is only positively related to emotional problems for boys. 


\section{Situational differences in oppositionality}

Second, we tested whether self-reports of oppositionality provide valid situationspecific information. Using MlWin, four multivariate regression analyses (Snijders \& Bosker, 1999) were conducted with self-reported oppositionality at home, at school and with peers as the dependent variables. In each of the four regression analyses, one of the criterion variables (e.g. conduct problems) was entered as the independent variable. The multivariate approach allows a formal comparison of the relations between oppositionality and criterion variables across situations and accounts for the correlations between the dependent variables, i.e. oppositionality at home, at school and with peers.

The regression models were built up in three consecutive steps. First, a baseline model was fitted in which one common regression equation was estimated for oppositionality at home, at school, and with peers. For each situation, the regression equation included one common intercept and the mean adult rating of a criterion variable (e.g. conduct problems). The baseline model embodies the assumption that the relation between oppositionality and the criterion variable concerned does not vary across situations or informants. Conceptually, the baseline model corresponds to the basic regression models used in the previous analyses.

In the second step, a model was fitted that allowed the regression lines for each situation to have different intercepts. The regression equation contained three separate intercepts (one for each situation) and the mean adult rating of the criterion variable. The model embodies the assumption that the mean oppositionality score differs across situations and that uniform relations exist with the criterion variable across situations.

In the third step, the slopes of the regression lines were also allowed to differ across situations. The regression equation contained three intercepts and three regression coefficients describing the relation between oppositionality and the criterion variable in each situation. According to this model, the relation between oppositionality and the criterion variable concerned varies across situations (but does not depend on the type of informant). The model examines the possibility that the strength of the relations with criterion variables differs across situations.

In the final step, the regression lines were allowed to differ across situations and informants, resulting in a total of six regression lines. This was achieved by estimating separate regression coefficients for parent and teacher ratings. The regression equation now contained three intercepts, three regression coefficients describing the relation between oppositionality and the parent rating of the criterion variable (e.g. conduct problems) in each situation, and three regression coefficients describing the relation between oppositionality and the teacher rating of the criterion variable in each situation. This model embodies the assumption that children provide unique information about their situation-specific behavior. If the estimation of separate regression coefficients for parent and teacher ratings results in a significantly better model fit, comparing these coefficients subsequently reveals whether 
oppositionality at home is more strongly related to parent ratings than to teacher ratings, and whether oppositionality at school is more strongly related to teacher ratings than to parent ratings.

Differences between the four regression models were evaluated by means of the likelihood ratio statistic (c.f. Goldstein, 1995). We tested whether each step in the modeling procedure resulted in a better fit than the model in the previous step. The analyses were done separately for boys and girls to avoid regression equations with too many interaction terms.

Table 2a.

Summary of the multivariate regression analyses for boys evaluating differential relations across situations and informants.

\begin{tabular}{|c|c|c|c|c|}
\hline Predictor variables & $\begin{array}{c}\text { Overall } \\
B(S E)\end{array}$ & $\begin{array}{l}\text { Home } \\
B(S E)\end{array}$ & $\begin{array}{l}\text { School } \\
B(S E)\end{array}$ & $\begin{array}{r}\text { Peers } \\
B(S E)\end{array}$ \\
\hline \multicolumn{5}{|l|}{ Conduct problems } \\
\hline Intercept & - & $1.98(.21)$ & $1.69(.20)$ & $1.61(.20)$ \\
\hline Parent rating & - & $.27(.09)$ & $.17(.09)$ & $.15(.09)$ \\
\hline Teacher rating & - & $.13(.08)$ & $.41(.07)$ & $.38(.08)$ \\
\hline \multicolumn{5}{|l|}{ Hyperactivity } \\
\hline Intercept & $1.42(.19)$ & - & - & - \\
\hline Mean adult rating & $.32(.04)$ & - & - & - \\
\hline \multicolumn{5}{|l|}{ Emotional problems } \\
\hline Intercept & $2.16(.18)$ & - & - & - \\
\hline Mean adult rating & $.24(.08)$ & - & - & - \\
\hline \multicolumn{5}{|l|}{ Prosocial behavior } \\
\hline Intercept & - & $5.47(.68)$ & $4.47(.68)$ & $4.81(.68)$ \\
\hline Parent rating & - & $-.19(.08)$ & $.01(.08)$ & $-.10(.08)$ \\
\hline Teacher rating & - & $-.18(.05)$ & $-.27(.05)$ & $-.21(.05)$ \\
\hline
\end{tabular}

Boys. Table 2a shows the parameter estimates of the best fitting regression models for boys. In the analysis with conduct problems as the criterion variable, two steps resulted in significant improvements. The baseline model was not significantly improved by estimating separate intercepts, but the subsequent addition of situation specific regression coefficients $\left(\Delta \chi^{2}(2)=6.73, p<0.05\right)$ and the estimation of separate regression coefficients for parent and teacher ratings $\left(\Delta \chi^{2}(3)=9.38, p<0.05\right)$ improved the fit of the model significantly. The multiple $R$ of the baseline model was .41. Adding separate regression coefficients to the model did not result in an increase in the multiple $R$, whereas the estimation of separate regression coefficients for parent and teacher ratings slightly increased the multiple $R$ to .42 . The regression coefficients in Table $2 \mathrm{a}$ show that self-reported oppositionality at home was more strongly related to parent ratings of conduct problems $(\beta=.27)$ than to teacher ratings $(\beta=.13)$, whereas self-reported oppositionality at school and with peers were more strongly 
related to teacher ratings $(\beta=.41$ and $\beta=.38)$ than to parent ratings $(\beta=.17$ and $\beta=.15)$. The top part of Figure 2a illustrates graphically how the relation between self-reported oppositionality and conduct problems differed across situations and informants. It can be seen that the regression line for parent ratings (dotted line) is above the averaged regression line (solid line) for oppositionality at home, whereas the regression line for teacher ratings (dashed line) is above the averaged regression line for oppositionality at school and with peers. Furthermore, the averaged regression line for oppositionality at home showed a less steeper slope than oppositionality at school and with peers (.20 in comparison to .29 and .27 ), indicating that the relation with conduct problems was less strong for oppositionality at home.

Figure 2a.

Regression lines for boys representing the relation of oppositionality with conduct problems and prosocial behavior across situations and informants.
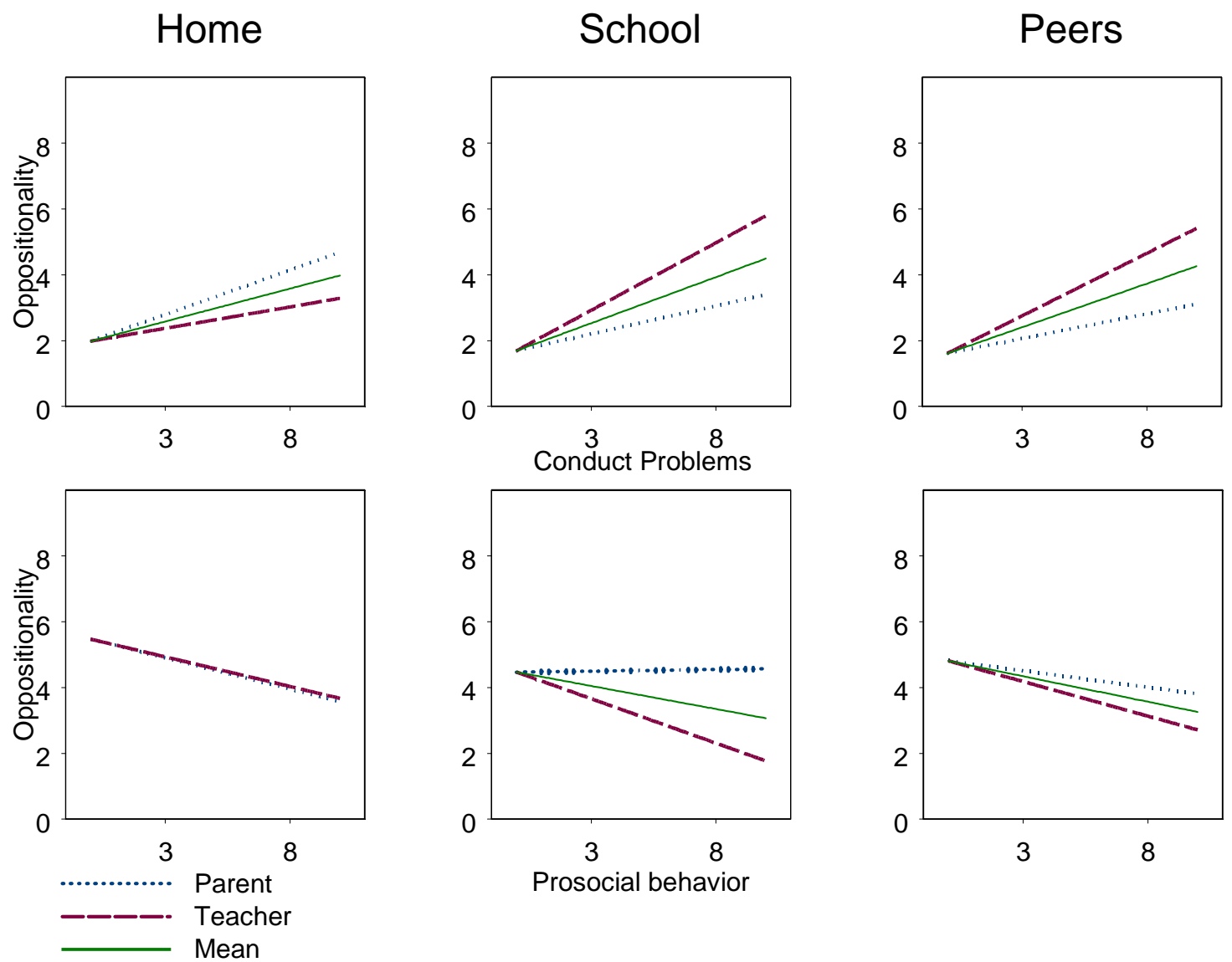

In the analyses with hyperactivity and emotional problems as criterion variables, none of the steps in our modeling procedure resulted in a significant improvement. The baseline models were consequently taken as the best models, implying that the relation of self-reported oppositionality with hyperactivity and emotional problems did not vary across situations or 
informants. Because the baseline model conceptually corresponds to the previous analysis, we refer to Figure 1 for a graphical illustration of these relations.

In modeling the relation between oppositionality and prosocial behavior, the only significant improvement in model fit was achieved by estimating separate regression coefficients for parent and teacher ratings $\left(\Delta \chi^{2}(3)=12.18, p<0.01\right)$. This step increased the multiple $R$ from .32 to .33 . The bottom part of Figure 2 a shows that self-reported oppositionality at home was similarly related to parent and teacher ratings $(\beta=-.19$ and

$\beta=-.18)$, whereas oppositionality at school and with peers were more strongly negatively related to teacher ratings $(\beta=-.27$ and $\beta=-.21)$ than to parent ratings of prosocial behavior $(\beta=.01$ and $\beta=-.10)$.

Table $2 b$.

Summary of the multivariate regression analyses for girls evaluating differential relations across situations and informants.

\begin{tabular}{lrrrr}
\hline & Overall & Home & School & Peers \\
Predictor variables & $B(S E)$ & $B(S E)$ & $B(S E)$ & $B(S E)$ \\
\hline Conduct problems & & & & \\
$\quad$ Intercept & - & $2.10(.19)$ & $1.18(.19)$ & $0.66(.15)$ \\
Parent rating & - & $.41(.11)$ & $.35(.10)$ & $.53(.08)$ \\
Teacher rating & - & $-.01(.11)$ & $.38(.11)$ & $.14(.09)$ \\
& & & & \\
Hyperactivity & & & & \\
$\quad$ Intercept & - & $2.52(.20)$ & $1.47(.20)$ & $.98(.16)$ \\
Mean adult rating & - & $.04(.06)$ & $.18(.06)$ & $.20(.05)$ \\
& & & & \\
Emotional problems & & & & \\
Intercept & - & $2.58(.21)$ & $2.03(.22)$ & $1.28(.17)$ \\
Parent rating & - & $.17(.07)$ & $.06(.07)$ & $.14(.06)$ \\
Teacher rating & - & $-.18(.07)$ & $-.16(.07)$ & $-.07(.07)$ \\
& & & & \\
Prosocial behavior & & & & \\
Intercept & - & $7.80(.92)$ & $6.37(.95)$ & $4.70(.78)$ \\
Parent rating & - & $-.47(.09)$ & $-.28(.09)$ & $-.19(.08)$ \\
Teacher rating & - & $-.13(.07)$ & $-.25(.07)$ & $-.19(.06)$ \\
& & & & \\
\hline
\end{tabular}

Girls. Table $2 \mathrm{~b}$ shows the parameter estimates of the best fitting regression models for girls. With conduct problems as the criterion variable, all steps resulted in significant improvements. The baseline model was significantly improved by estimating separate intercepts $\left(\Delta \chi^{2}(2)=67.16, p<0.01\right)$, separate regression coefficients for each situation $\left(\Delta \chi^{2}\right.$ $(2)=6.33, p<0.05)$ and separate regression coefficients for each informant $\left(\Delta \chi^{2}(3)=17.88\right.$, $p<0.01$ ). The multiple $R$ of the baseline model was .35, whereas the multiple $R$ of the final model was .39. The regression lines for each situation depicted in the top part of Figure $2 \mathrm{~b}$ show that oppositionality at home was more strongly related to parent ratings $(\beta=.41)$ than to teacher ratings $(\beta=-.01)$ of conduct problems. The relation between oppositionality and 
conduct problems did not appear to vary across informants for oppositionality at school ( $\beta=.35$ and $\beta=.38$ ), whereas oppositionality with peers appeared to be more strongly related to parent ratings $(\beta=.53)$ than to teacher ratings of conduct problems $(\beta=.14)$. Furthermore, the averaged regression line (solid line) for oppositionality at home showed a less steeper slope than oppositionality at school and with peers (.20 in comparison to .36 and .33 ), indicating that the relation with conduct problems was less strong for oppositionality at home.

With hyperactivity as the criterion variable, the baseline model was significantly improved by estimating separate intercepts $\left(\Delta \chi^{2}(2)=67.16, p<0.01\right)$ and separate regression coefficients $\left(\Delta \chi^{2}(2)=6.93, p<0.05\right)$. These steps increased the multiple $R$ from .16 to .19 . The relation between oppositionality and hyperactivity appeared to vary across situations only. The regression coefficients in Table $2 b$ indicate that oppositionality at home was not related to the mean adult rating of hyperactivity $(\beta=.04)$, whereas oppositionality at school and with peers were related to the mean adult rating of hyperactivity $(\beta=.18$ and $\beta=.20)$.

With emotional problems as the criterion variable, the baseline model was significantly improved by estimating separate intercepts $\left(\Delta \chi^{2}(2)=67.16, p<0.01\right)$ and separate regression coefficients for parent and teacher ratings in each situation $\left(\Delta \chi^{2}(3)=9.79, p<0.05\right)$. The multiple $R$ was increased from .08 to .20 . The middle part of Figure $2 \mathrm{~b}$ shows that in all three situations oppositionality was differentially related to parent and teacher ratings. Oppositionality appeared to be moderately positively related to parent ratings of emotional problems $(\beta=.17, \beta=.06$, and $\beta=.14)$, but negatively related to teacher ratings of emotional problems $(\beta=-.18, \beta=-.25$, and $\beta=-.07)$.

In the analyses with prosocial behavior as the criterion variable, the baseline model was significantly improved by adding separate intercepts $\left(\Delta \chi^{2}(2)=67.16, p<0.01\right)$ and situation-informant interactions $\left(\Delta \chi^{2}(3)=10.45, p<0.05\right)$. The multiple $R$ of the baseline model was .35 and the multiple $R$ of the final model was .37 . The bottom part of Figure $2 \mathrm{~b}$ depicts the separate regression lines for parent and teacher ratings in each situation. It can be seen that oppositionality at home was more strongly (and negatively) related to parent ratings $(\beta=-.47)$ than to teacher ratings of prosocial behavior $(\beta=-.13)$. The relation between oppositionality and prosocial behavior did not appear to differ in the school situation $(\beta=-.28$ and $\beta=-.25$ ) and in the situation with peers $(\beta=-.19$ and $\beta=-.19)$. 
Chapter 3

Figure $2 \mathrm{~b}$.

Regression lines for girls representing the relation of oppositionality with conduct problems, emotional problems and prosocial behavior across situations and informants.
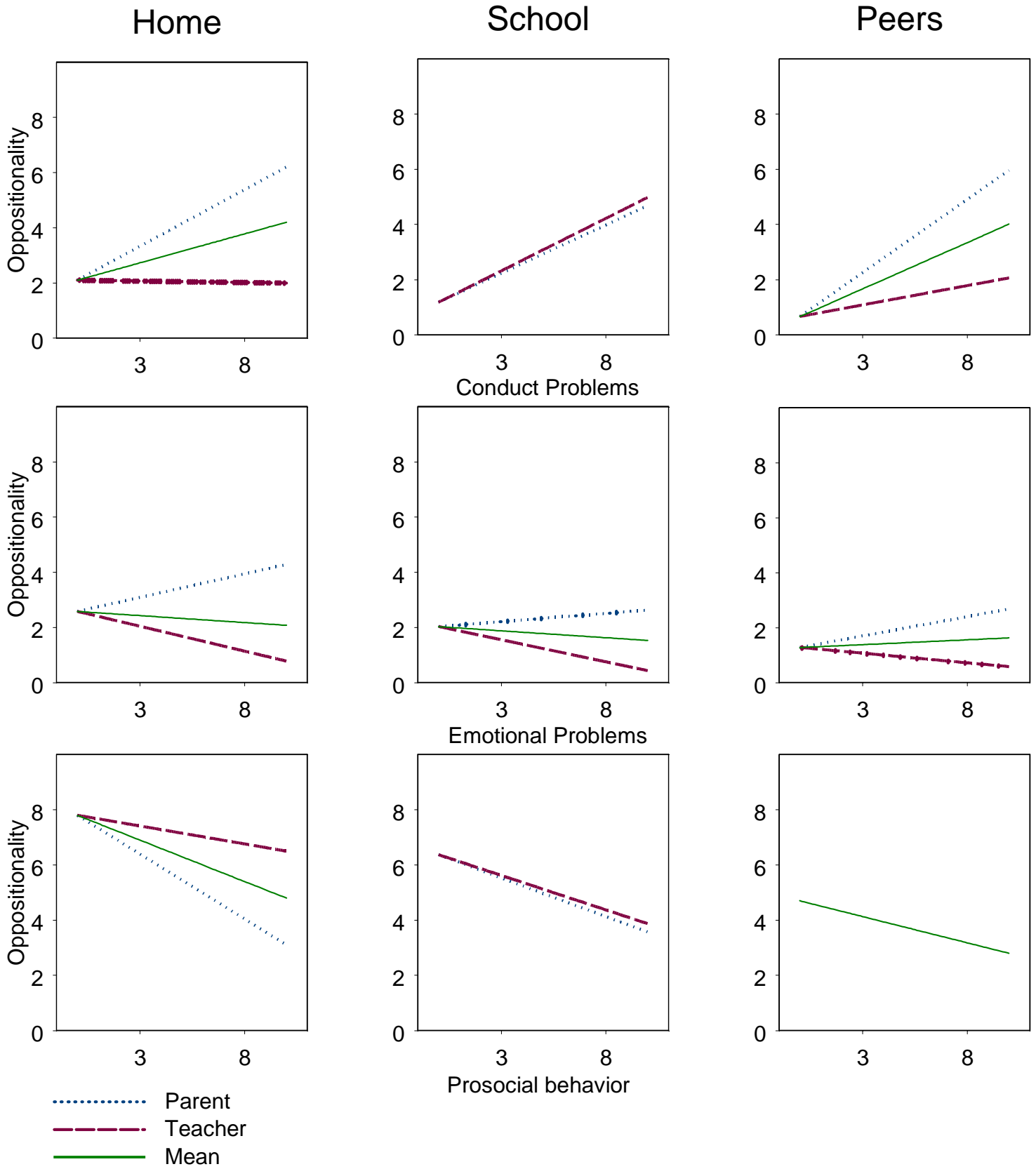


\section{Discussion}

This study examined the validity of children's self-reported oppositionality across situations. Self-reports of oppositionality in situations with parents, teachers, and peers were obtained by means of the Amsterdam Scale of Oppositionality (ASO) and related to independently rated criterion behaviors. In judging the validity of self-reported oppositionality as obtained by the ASO, several findings are salient. First, self-reported oppositionality appeared to be related in theoretically meaningful ways to parent and teacher ratings of children's psychopathology and social functioning. In line with our expectations, self-reports appeared to be positively related to adult ratings of children's conduct problems and hyperactivity, and negatively to their ratings of children's prosocial behavior. Consistent with previous research on gender differences in the co-morbidity between disorders (Moffitt et al., 2001), boys and girls were very similar with regard to their pattern of associated behaviors. However, gender differences did emerge for the relations with hyperactivity and emotional problems. Oppositionality appeared to be less strongly related to hyperactivity for girls and the expected positive relation between oppositionality and emotional problems was only observed for boys.

For a proper appraisal of these findings, the size of the associations found can best be compared with the magnitude of correlation coefficients in studies with the same area of investigation and similar measurement methods (Hemphill, 2003). Therefore, we choose the typical correlation between self-reports and adult ratings of children's behavioral problems as a benchmark to interpret the correlations in the present study. An extensive meta-analysis of cross-informant correlations revealed a mean correlation between self-reports and adult ratings of .22 , but this correlation was somewhat higher for preadolescent children and for externalizing behaviors (Achenbach et al., 1987). Contrasting this correlation between ratings of the same construct with the correlations observed in the present study between selfreported oppositionality and parent and teacher ratings of conduct problems (.41), prosocial behavior (-.36) and hyperactivity (.42), indicates a substantial criterion-related validity of selfreported oppositionality as obtained by the ASO.

The criterion-related validity of the ASO appears to be at variance with research that has questioned the validity of self-report instruments (e.g. Handwerk, Larzelere, Soper, \& Friman, 1999; Loeber, Green, Lahey, \& Stouthamer-Loeber, 1991). There are some features of the ASO that have possibly contributed in a positive way to its validity. First, the ASO intends to measure the continuum of oppositionality and differs in this respect from other instruments with a clear clinical perspective. Possibly, the descriptions of everyday interactions with parents, teachers, and peers in the ASO provide more valid responses than asking children about the frequency and intensity of clinical symptoms. A second positive feature of the ASO is the facet approach that certified a systematic sampling of children's 
responses in different situations. Finally, the forced-choice format of the ASO may have contributed to its validity by reducing errors as a result of response sets. By presenting two equally socially undesirable alternatives (one that is almost too well-behaved, and one that conflicts with a social rule) children's choice for either the oppositional or the nonoppositional alternative may be more in accordance with their true feelings and behaviors. Furthermore, the forced-choice format may be effective in minimizing errors as a function of response sets by masking the scoring of children's responses (Bownas \& Bernardin, 1991). These are obviously speculations, but some support for a lower susceptibility to faking for the forced-choice format can be found in the literature (Jackson, Wroblewski, \& Ashton, 2000).

The results further show that self-reported oppositionality as obtained by the ASO reflects valid information on situational differences in the child's behavior. In line with our expectations, children's responses in interactions at school provided more valid information than their responses in interactions at home or with peers. Oppositionality at school was more strongly related to conduct problems than oppositionality at home and with peers. In addition, the analyses revealed that for girls oppositionality at school and in situations with peers were related to hyperactivity, but oppositionality at home was not related to hyperactivity. No differential relations across situations were found for emotional problems and prosocial behavior. The differential relations between oppositionality and other disruptive behaviors across situations support the assumption that the school context triggers oppositional responses to a greater extent than the family context.

As far as we know, the current study is the first to explore the notion that oppositionality in some situations is more strongly linked to forms of psychopathology than in other situations. Drabick, Strassberg, and Kees (2001) have shown that the relation of noncompliance with aggression and social adjustment depends on the specific behavior that the child displays (e.g. a verbal, emotional or physical response), but we know of no studies that have examined the possibility that relations between oppositionality and its correlates depend on the situation in which children display oppositionality.

The observed differential relations across situations indicate that distinguishing between different situations can advance our understanding of oppositionality and provide insights that have direct relevance for clinical practice and future research efforts. Our results suggest that situational information may help clinicians to assess the severity of the child's oppositional problems. To ensure the clinical implications of our findings, a post-hoc analysis was conducted focusing on the mean adult conduct problems rating of those who scored in the clinical range on oppositionality at home, at school, and in both situations (the 80th percentile). Children with oppositional problems at home had the lowest conduct problems ratings $(M=1.34, S D=1.16, N=53)$, although their scores were still higher than those of "normal" children $(M=1.05, S D=1.16, N=340)$. Children with oppositional problems at school were rated higher than those with home problems only $(M=1.87, S D=1.42, N=59)$. In addition, those with a cross-situational pattern of oppositionality (both home- and school problems) 
were rated the highest on conduct problems $(M=2.35, S D=1.72, N=53)$. These results corroborate the observed differential relations and again strongly suggest that different situational profiles indicate different levels of severity. Furthermore, in line with clinical diagnostic systems, cross-situational consistency appears to be most indicative of child psychopathology.

With respect to future research efforts, the observed differential relations across situations suggest that we may have to reevaluate traditional measurement approaches. It is generally assumed that oppositional behaviors in different situations can be regarded as repeated measures of the same general oppositional tendency, but the present study suggests that responses from different situations may not be equivalent measures. Second, the findings illustrate the need to determine which behaviors are more normative and which behaviors clearly reflect deviancy (Wright \& Zakriski, 2003). Future studies could help to resolve this more general issue by relating situation-specific information to social adjustment criteria or negative developmental outcomes and consequently clarify in which situations the child's behavior can possibly be regarded as more or less normative.

Unique relations of oppositionality at home and at school with parent and teacher ratings of the criterion variables provided further support for the assumption that children's responses across situations reflect valid information about situational differences in oppositionality. For conduct problems, oppositionality at home appeared to be more strongly related to parent ratings than to teacher ratings (for boys and girls). Oppositionality at school appeared to be more strongly related to teacher ratings (for boys only). The analyses also revealed that the relation between oppositionality and prosocial behavior varied across situations and informants. The finding that parallel measures (e.g. self-reports of home behavior and parent ratings) showed the greatest criterion-related validity is perhaps not surprising, but underscores the situational specificity of oppositionality. The results extend the previous finding that children's oppositionality at home is only moderately related to oppositionality at school (Hoffenaar \& Hoeksma, 2002) by showing that children can provide unique information about their behavior in these situations. Adult informants appear to have only limited access to each of these situations.

Theoretically, the situational specificity of children's responses illustrates that oppositionality does not occur within the vacuum of the individual (Alexander \& Pugh, 1996), but within relationships with parents, teachers and peers. Situational differences in children's oppositionality do not appear to be random variation, but appear to be differentially related to conduct problems and appear to be linked to the observations by the persons directly involved in oppositionality, i.e. parents and teachers. A child displaying oppositionality at home appears to be different from a child displaying oppositionality at school. This conclusion is in line with a transactional conceptualization of oppositionality (Greene \& Doyle, 1999). From a transactional perspective, oppositionality is not just the 
result of specific child characteristics, but is also determined by situational demands and relational processes.

Although many clinicians may be convinced of the importance of parents, schools, and peers, current diagnostic instruments seem to provide little help in recognizing environmental or relational influences. Information about the situational aspects of oppositionality obtained by means of self-reports may therefore provide a useful addition to the current range of diagnostic tools. Increased attention to the situational aspects of oppositionality can help clinicians to recognize the absence or presence of relational factors in the diagnosis of ODD. Situation-specific oppositionality may point to relational difficulties or family dysfunction, whereas cross-situational consistency may point to compromised skills in the domains of emotion regulation or problem solving.

The findings further point to the possibility of developing psychometrically sound instruments that take full advantage of the child's unique position to report on their behavior in interactions with various relationship partners. It has long been recognized that children's self-reports reflect a compilation of their behavior at home, at school, and with peers (Phares, Compas, \& Howell, 1989), but the present study suggests that self-reports can also provide valid information about the patterning of responses across these different situations. Smith, Pelham, Gnagy, Molina, and Evans (2000) already noted the potential usefulness of selfreport ratings of specific situations in which children interact with specific adults, but we know of no studies that have acted upon their recommendation.

The failure to take full advantage of child self-reports is rather surprising given the fact that previous research by Wright and Zakriski $(2001,2003)$ has shown that groups of externalizing children are characterized by considerable within-group heterogeneity in both their reactions to events and the responses those reactions in turn evoke from others.

The authors conclude that traditional syndromal measures may obscure important differences in the quality of children's social interactions and underscore the need to develop instruments that are sensitive to situational differences in the child's behavior. The present study suggests that self-reports should be considered as a serious option to incorporate the situational aspects of children's behavior more explicitly into assessment and measurement procedures.

Several limitations of the present study should be addressed. First, the validation of children's responses across situations was partly based on the assumption that differences between parent and teacher ratings reflect true differences between children's behavior at home and at school. Although there is now some empirical support for this assumption (Burns et al., 2003), some may argue that differences between parent and teacher ratings solely reflect the unique perceptions of adult informants. Parent ratings may for example be more affected by socially desirable responses, whereas teacher ratings may be influenced by the other children in their classroom (Chan, Ramey, Ramey, \& Schmitt, 2000). However, the present findings contradict a systematic informant bias in one direction. First, the parent- 
teacher difference in the relation between oppositionality and conduct problems varied across situations and gender. Second, differential relations between parent and teacher ratings were not observed consistently for all criterion behaviors. Differential relations were most consistently observed for those behaviors that contain a clear interpersonal component, i.e. conduct problems and prosocial behavior. We consequently conclude that although the current study was not intended to resolve the issue of why parents and teachers provide different information, our findings are most consistent with the assumption that differences between these informants reflect true situational differences.

A main limitation is that the current study lacks important independent measures that would have contributed to a more comprehensive validation of the ASO. The validation of situational differences in self-reported oppositionality would have benefited substantially from the inclusion of parent and teacher ratings of oppositionality or direct observations of oppositionality in different situations. Given that this information was not available, the lack of peer ratings of criterion behaviors remains a serious limitation. As a consequence, we could not fully demonstrate the validity of oppositional responses in situations with peers.

Finally, it is unclear to what extent the conclusions generalize to the general population. Although the results suggest that the rather high nonresponse rates for parent and teacher reports did not result in bias, the question of whether the sample adequately reflected the general population remains unanswered. It is also unclear to what extent the conclusions generalize to clinical samples. Although future research is needed to determine the value of self-reported oppositionality in clinical samples, recent research by Friman et al. (2000) showed that the DISC provided valid information on conduct problems and oppositionality for a group of children entering residential care. This suggests that self-reports of disruptive behaviors can also be useful in clinical practice.

Despite the possible limitations, the results from the present study indicate that selfreported oppositionality as obtained by the ASO can provide valid information. In addition, situational differences in children's responses also appear to be valid. Therefore, the ASO seems able to provide direct information from the child itself about the overall level of oppositionality as well as more detailed information about the situational aspects of the child's oppositionality. The information on situational differences can be helpful in future research efforts that aim to further our understanding of how factors within the child, factors within the environment and the dynamic interplay between child and environmental factors contribute to the development of oppositionality.

In clinical practice, collecting data from multiple sources is becoming a standard procedure. Clinical practice guidelines recommend that one should investigate whether discrepancies between informants reflect true situational differences (Achenbach, 1993), but provide the clinician with little clues on how to disentangle discrepancies due to different perspectives from discrepancies due to situational differences. Self-report instrument like the ASO might provide the clinician with the information needed, especially for externalizing 
problems. If the discrepancies between adult informants are largely consistent with the situational differences in children's responses across different situations, this would strongly point in the direction of situational-specificity in the child's behavior. If the child is referred for oppositional problems, self-reports might even be considered as a starting point of the diagnostic process. Depending on the presence of situational differences in children's own responses, the clinician could subsequently decide whether to collect additional data from teachers, parents or peers or only a subset of these informants. Given the need for costeffective assessment procedures (Wood, Garb, Lilienfeld, \& Nezworski, 2002), the use of self-reports as an initial screening for situational differences may be a promising new approach. 
Self-reported oppositionality 


\section{CHAPTER 4}

\section{Understanding informant disagreement on children's conduct problems}

Hoffenaar, P. J., \& Hoeksma, J. B. (in revision). Understanding informant disagreement on children's conduct problems. Psychological Assessment 
Clinicians and researchers generally accept the need to gather information about children's functioning from different sources in diverse situations. To assess a child's conduct problems it is essential to establish impairment in the child's functioning at school, at home or in peer relationships (Hinshaw \& Anderson, 1997). Because a "gold standard" or single criterion situation against which to validate informant's reports is typically lacking (Achenbach, 1978; Baillargon et al., 2001), each informant is assumed to contribute valid information about the child. Unfortunately, most studies have found only low to moderate correlations between informant reports (Achenbach et al., 1987; Angold \& Costello, 1996; Fisher \& Fagot, 1996; Loeber, Green, Lahey, \& Stouthamer-Loeber, 1991a; Offord et al., 1996). The limited convergence between informant's reports has led some researchers to search for optimal (combinations of) informants for the behavior concerned (Angold \& Costello, 1996; Hart, Lahey, Loeber, \& Hanson, 1994; Loeber et al., 1991a). Others argue that we need to understand why informants disagree (Fisher \& Fagot, 1996; Kuo, Mohler, Raudenbush, \& Earls, 2000).

The search for optimal informants is based on the assumption that some behaviors are more validly reported by some informants than others (Hart et al., 1994). The optimal informant is determined by relating informants' judgments to psychiatric diagnoses or other criterion variables. However, these criteria are also dependent on the person making the judgment and may therefore be less suited to validate informants' judgments. Furthermore, searching for optimal informants neglects the possibility that each informant contributes different, but valid information (Achenbach et al., 1987). For instance, teacher reports on conduct problems may provide valid information on the child's deviant behavior at school, whereas parent reports may provide valid information on the child's compliance to parental requests. If each informant contributes unique valid information, the search for optimal informants could be misleading.

Given the possibility of different but valid information in informants' ratings, and the absence of definitive criteria against which to validate informants' reports, our attention should shift towards understanding why informants disagree. Although a number of factors affecting agreement have been proposed (Greenbaum, Decrick, Prange, \& Friedman, 1994; Grills \& Ollendick, 2002), few studies have directly examined their effects (Youngstrom, Loeber, \& Stouthamer-Loeber, 2000). The present study aims to provide a better understanding of the factors influencing informants' ratings, by focusing on parent- and teacher ratings of preadolescents' conduct problems in the general population.

\section{Agreement on conduct problems}

Conduct problems refer to behaviors that violate basic social rules. The domain of conduct problems ranges from disobedience, temper tantrums and fighting to more serious antisocial acts such as lying and stealing. Conduct problem behaviors can be seen as more extreme manifestations of oppositionality (Rey et al., 1988). Either a symptoms-based 
diagnostic approach or a psychometric approach can be used to assess conduct problems (Frauenglass \& Routh, 1999). In the present study we focus on information obtained by means of psychometric instruments. More specifically, we focus on agreement between parent and teacher reports of the child's conduct problems, rated by means of the Strength and Difficulties Questionnaire (SDQ; Goodman, 1997).

Several studies have shown that agreement between parent- and teacher ratings on conduct problems is generally low. Youngstrom, Loeber, \& Stouthamer-Loeber (2000) reported a correlation of .26 between parent and teacher ratings of conduct problems and Stormshak and Webster-Stratton (1999) reported a correlation of .22. In studies by Fergusson and Horwood $(1987,1989)$, correlations ranged from .33 to .40 . Their findings are in line with the mean correlation between parent and teacher ratings of externalizing problems, as summarized in an extensive meta-analysis of cross-informant correlations by Achenbach, McConaughy and Howell (1987). The problem of low agreement is also pertinent with regard to the main instrument of the present study, the SDQ. In a large-scale nationwide sample of 515 year-old British children, the correlation between parent and teacher ratings of children's conduct problems was .37 (Goodman, Ford, Simmons, Gatward, \& Meltzer, 2000). Although it was concluded that, at least for conduct problems, inter-informant agreement for the SDQ was substantially better than the average level of agreement for other measures, this correlation still reveals a distressing lack of agreement between parents and teachers. The problem then is to account for this correlation reflecting low agreement.

\section{Understanding informant disagreement}

The lack of agreement between parents and teachers can possibly best be understood by using the psychometric model proposed by Fergusson and Horwood $(1987,1989)$. This model partitions informants' ratings in three components: (1) the true score, (2) the unique informant score and (3) the random error score. The true score reflects the child's generalized behavioral tendency to display conduct problems. The true score has also been referred to as the trait score, because traits are often conceptualized as general behavioral tendencies (Wright, Zakriski, \& Drinkwater, 1999). The unique informant score reflects source effects arising from situational factors and informant factors. The random error score contains both systematic and nonsystematic error. The first is a reliable residual score specific to the particular measure, whereas the latter is nonsystematic measurement error due to unreliability of the informants and instruments involved (Lance, Noble, \& Scullen, 2002). The true score, the unique informant score and the error score are assumed to be uncorrelated.

According to this model, agreement between parents and teachers is accounted for by the child's generalized behavioral tendency as observed by both parents and teachers. The unique scores of parents and teachers reflect the part of their ratings that is specific to that source of information. The unique informant scores may contain valid situation-specific information about the child's behavior or the unique perspective of the informant. Both of 
these source effects attenuate agreement. Finally, random measurement error decreases the reliability of parent and teacher ratings and thus affects agreement negatively.

\section{Source effects}

Source effects may be the result of (1) situational specificity of the child's behavior and (2) informant characteristics. The effect of situational specificity on measures of children's behavior has been recognized for a long time (Achenbach, 1978). Situational differences in children's behavior may be partly due to developmental processes. Conduct problems may first appear in the family, before they generalize to other situations (Stormshak \& Webster-Stratton, 1999). Another reason for the situational specificity of children's behavior is that the characteristics of the situation (home and school) and the characteristics of the relationships (with teachers and parents) in the various contexts differ. These differences are likely to affect children's display of conduct problems (Hope \& Bierman, 1998; Stormshak \& Webster-Stratton, 1999). The display of conduct problems is therefore apt to vary across situations, although cross-situational relations between children's behavior naturally exist. Source effects can be due to the situational specificity of conduct problems, because informants typically observe children only in specific situations. Parents and teachers may provide valid information about the child's functioning in specific situations that does not necessarily overlap.

A major obstacle in testing whether source effects reflect true situational differences has been to obtain situation-specific information that can account for source effects in informants' ratings. To examine the effect of situational specificity of children's behavior on informants' ratings, information is needed about the child's behavior in specific situations. Gomez, Burns, Walsh, and Moura (2003) recently suggested to use direct observations of home and school behavior as external criteria that could differentially predict the source effects in parent and teacher ratings. In the present study, we examined whether children's self-reports on their behavior at home, at school, and with peers differentially predict source effects in parent and teacher ratings.

Source effects can also be due to informant characteristics. Informants do not only differ in their opportunities for observing children, but may also interpret their observations differently (Achenbach et al., 1987). Parents and teachers have different perspectives on children's behavior. Differing perceptions may relate to different tolerance levels and norms with regard to conduct problems. We focus on the implicit norm of the teacher as an important informant characteristic that may account for source effects in teacher ratings.

Teachers' norms with regard to conduct problems are likely to be affected by the school environment and teachers' personal attitudes towards children's problem behavior. Teachers will consequently differ in their tolerance level of conduct problems. The findings of Eddowes, Aldridge and Culpepper (1994) with respect to hyperactivity may serve as an example. They showed that teacher ratings were affected by teachers' preference for 
structured versus unstructured classrooms. Similarly, teacher ratings of children's conduct problems are possibly affected by their norms and standards with regard these disruptive behaviors.

In sum, source effects may be due to situational differences in the child's behavior and norms and standards with regard to disruptive behaviors. Source effects due to situational differences in the child's behavior would indicate that each informant provides different, but valid information. Source effects in teachers' ratings due to different implicit norms between teachers indicate that teachers and parents employ different strategies or methods in rating children, implying bias or measurement artifacts in teachers' ratings. Therefore, source effects can represent accuracy, bias, or a combination of accuracy and bias. The possibility that source effect represent a combination of accuracy and bias has generally been noted as the most likely one (DuPaul, 2003; Gomez, Burns, Walsh, \& Moura, 2003b; Greenbaum et al., 1994), but empirical investigations of the variables determining source effects are very limited. The current study investigates situational and informant variables that are thought to determine source effects in parent and teacher ratings of externalizing problems. As a consequence, the study also investigates whether source effect in informants' ratings reflect accuracy, bias or a combination of both.

\section{Random measurement error}

Besides situational specificity and informant characteristics, agreement is also affected by unreliability of informants' ratings. Unreliability may depend on the visibility of the target behavior being assessed. Conduct problems are likely to attract the attention of both parents and teachers and may be more easily noticed than for instance symptoms of depression and anxiety (Youngstrom et al., 2000). But not all conduct problems are disruptive to family- or classroom functioning. Covert conduct problems lack this disruptive effect. Lying and theft are considered covert conduct problems, because these acts are performed secretly (Frick et al., 1993; Loeber et al., 1991b). Covert conduct problems are harder to detect and information concerning these behaviors is not necessarily shared between informants. Thus, the limited agreement between parents and teachers on conduct problems may be partly due to unreliability in their ratings of behaviors that largely go unnoticed.

In sum, there are several sources that may account for the generally low agreement between informants. It is suggested that agreement between parents and teachers on conduct problems is affected by (1) situational specificity of the child's behavior, (2) different implicit norms of the teachers and (3) the lack of visibility of more covert problem behaviors. Although these factors have been discussed in the literature (Greenbaum et al., 1994; Grills \& Ollendick, 2002; Rowe \& Kandel, 1997), they have not been investigated empirically. 


\section{Methodological problems}

A first problem is to disentangle source effects and measurement error. A second problem is to determine the separate effects of situational specificity and informant characteristics on source effects and to determine the effect of visibility on measurement error. Bollen and Paxton (1998) recently proposed a structural equation model to separate source effects from measurement error. They also suggested a method to explain source effects.

In the present study, the method proposed by Bollen and Paxton (1998) was applied to parent and teacher ratings of conduct problems obtained by means of the SDQ. The method is based on a factor-analytic model, containing one general factor and two unique informant factors. In the model, all conduct problem items load on the general factor. Each item also loads on either the unique parent factor or the unique teacher factor (see Figure 1). The general factor corresponds to the agreement between parents and teachers, whereas the unique factors correspond to source effects. The reliability is inferred from the factor loadings of the items on the general factor (Bollen, 1989). Finally, the unique factors (source effects) are thought to be determined by situational and informant variables.

A major obstacle in studies on informant disagreement has been to obtain situationspecific information that can account for source effects in informants' ratings. This problem was solved by using information from the children rated. Children provided information concerning their oppositional responses in different situations by filling out the Amsterdam Scale of Oppositionality (ASO). In determining the structural validity of this scale, Hoffenaar and Hoeksma (2002) showed that oppositionality is to a large extent situation-specific.

\section{Method}

\section{Participants}

The target sample consisted of 1,194 children, their parents, and 54 teachers in 20 Dutch primary schools. Schools were selected from a register of all Dutch primary schools. A nation-wide self-weighting cluster sample was drawn. Probability of inclusion for each school was inversely proportional to the mean school size per province (data on school size were provided by the Dutch Central Bureau for Statistics, CBS, 1997). Parents and teachers were asked to rate the children by means of the SDQ as part of a study to assess the psychometric qualities of the Amsterdam Scale of Oppositionality (ASO; Hoffenaar \& Hoeksma, 2002). Children were asked to fill out the ASO and the self-report version of the SDQ. Due to nonresponse of the teachers $(34.1 \%)$, parents $(32.5 \%)$, and children involved $(2.6 \%)$ the target sample was reduced by $57.7 \%$. Thus complete records were obtained for 505 children ( $M$ age $11.31 \mathrm{yr}(S D=.90)) ; 251$ boys and 254 girls $)$.

The effect of nonresponse could be examined, because self-report information was available for most of the excluded cases. We compared the 505 participants included in the final sample and the 689 participants who were left out due to missing data or nonresponse by 
parents and teachers on their level of self-reported oppositionality. The mean generalized oppositionality score was $6.82(S D=5.43)$ for the children in the final sample and 7.82 $(S D=5.61)$ for the nonresponse group. Although those who were not included in the final sample had significantly higher oppositionality scores, the difference between the two groups of participants accounted for less than $1 \%$ of the variance. Because the actual amount of bias depends on the nonresponse rate and the effect size of the difference, the extent to which the observed difference introduced bias can be calculated more precisely by applying the formula presented by Gerrits, van den Oord, and Voogt (2001). The bias was .006, indicating that the difference between the "observed" mean in the final sample of participants and the true "unobserved" mean in the total sample is negligible.

\section{Procedure}

A research assistant or the first author visited the participating schools. The ASO and the SDQ were group administered to the children of the 6th, 7th and 8th grades. Teachers received SDQ questionnaires for each child in their class and a return envelope. Parents were contacted through their children; the participating children received a return envelope containing the SDQ with the instruction to give this envelope to their parents.

\section{Conduct Problems}

The conduct problems scale of the Strength and Difficulties Questionnaire (SDQ; Goodman, 1997) contains five items (displayed in Table 1). Note that none of the items specifically refer to home behavior or classroom behavior and that the wording is identical for the parent- and teacher version. Each item is scored 0 if "not true" is marked by the respondent, 1 for "somewhat true" and 2 for "certainly true".

Table 1. Item content of the SDQ Conduct Problems Scale

\begin{tabular}{lll}
\hline Variables & Item & \multicolumn{1}{c}{ Conduct problems } \\
\hline CD1 & SDQ5 & Often has temper tantrums or hot tempers \\
CD2 & SDQ7 & Generally obedient, usually does what adults request \\
CD3 & SDQ12 & Often fights with other children or bullies them \\
CD4 & SDQ18 & Often lies or cheats \\
CD5 & SDQ22 & Steals from home, school or elsewhere \\
\hline
\end{tabular}

The psychometric properties of the SDQ were recently investigated in a large representative sample (Goodman, 2001). Factor analysis of the SDQ revealed that the items of the SDQ loaded primarily on the predicted factors. Support for the predicted factor structure of the SDQ was also found in a large sample of Dutch children and adolescents (Muris et al., 2003). In addition, both studies indicated that the internal consistency and test-retest stability of the scales were acceptable. The concurrent validity of the SDQ scales was also examined. 
In the study by Goodman, the conduct problems scale of the parent and teacher version of the SDQ was found to predict the relevant DSM-IV diagnoses (ODD, CD) with high specificity.

In the present sample, the Cronbach's alphas for the conduct problems scale were .56 $(95 \%$ C.I. $=.50-.62)$ for the parent ratings, and $.73(95 \%$ C.I. $=.70-.77)$ for the teacher ratings. The confidence intervals cover the values reported by Goodman (Goodman, 2001).

\section{Situational variables: Oppositionality}

Situation-specific information was obtained by using the ASO. The ASO assesses children's self-reports on their oppositionality in multiple situations. The ASO was constructed according to a facet-design (Fiske, 1971). A response facet and a situational facet defined the content domain of oppositionality. The items of the ASO represent combinations of the response facet elements (behaviors and emotions) and the situational facet elements (home, school and situations with peers). Each combination is represented by five forcedchoice items, resulting in a total of 30 items. Each item consists of a description of the situation, followed by two response alternatives: one oppositional alternative and one nonoppositional response alternative. These alternatives were either emotional responses or behavioral responses.

An example of an item reflecting a behavioral response in interactions with parents is (with assigned scores between brackets): When my dad or mum tells me to help, I do it (0)/I pretend I haven't heard (1). An item reflecting oppositional feelings towards the teacher is: When the teacher punishes me, I feel sad (0)/I get angry (1). An item reflecting an emotional response in situations with peers: When another child is looking for trouble, I feel like fighting (1)/I want to walk away (0).

The validity of the ASO has been supported in two studies. In the first, cross sectional study of 1,158 children, we examined the structural validity of the ASO using confirmatory factor analyses (Hoffenaar \& Hoeksma, 2002). Supportive evidence was found for a structural model consisting of interrelated emotional and behavioral response factors and three mutually related situational factors. The factors representing the response tendencies and the factors representing the situational effects appeared to be independent. Because oppositionality appeared to be a combination of two independent elements, aggregation over responses within situations is justified.

Scores for oppositionality in specific situations are obtained by summing the responses within that specific situation. This results in scores for oppositionality at home, at school and in situations with peers that each range from 0 to 10 . In this study, the Cronbach's alphas for oppositionality at home, at school and with peers were $.70(95 \%$ C.I. $=.67-.72), .78(95 \%$ C.I. $=.76-.80)$ and $.75(95 \%$ C.I. $=.73-.77)$, respectively. 


\section{Informant variables: Teacher's norms}

Individual teachers' mean Conduct Problems rating was taken as an indication of their internal standards towards conduct problems. For boys and girls separately, the teacher's mean judgment was calculated across the children rated by this teacher (Note: teacher means were calculated for all children in their class, including children for whom parental information was unavailable).

\section{Results}

To determine the effects of the situational specificity of the child's behavior, implicit norms of the teacher and the lack of visibility of covert problem behaviors, we first determined the amount of variance due to trait, source and error effects by fitting the threefactor model proposed by Bollen and Paxton (1998) to the data. Various alternative models have been described to estimate trait, source and error effects in informants' ratings. Each of these models seems to have strengths and weaknesses (Eid, Lischetzke, Nussbeck, \& Trierweiler, 2003), but because of our interest in determining and explaining source effects in both parent and teacher ratings the model depicted in Figure 1 was chosen.

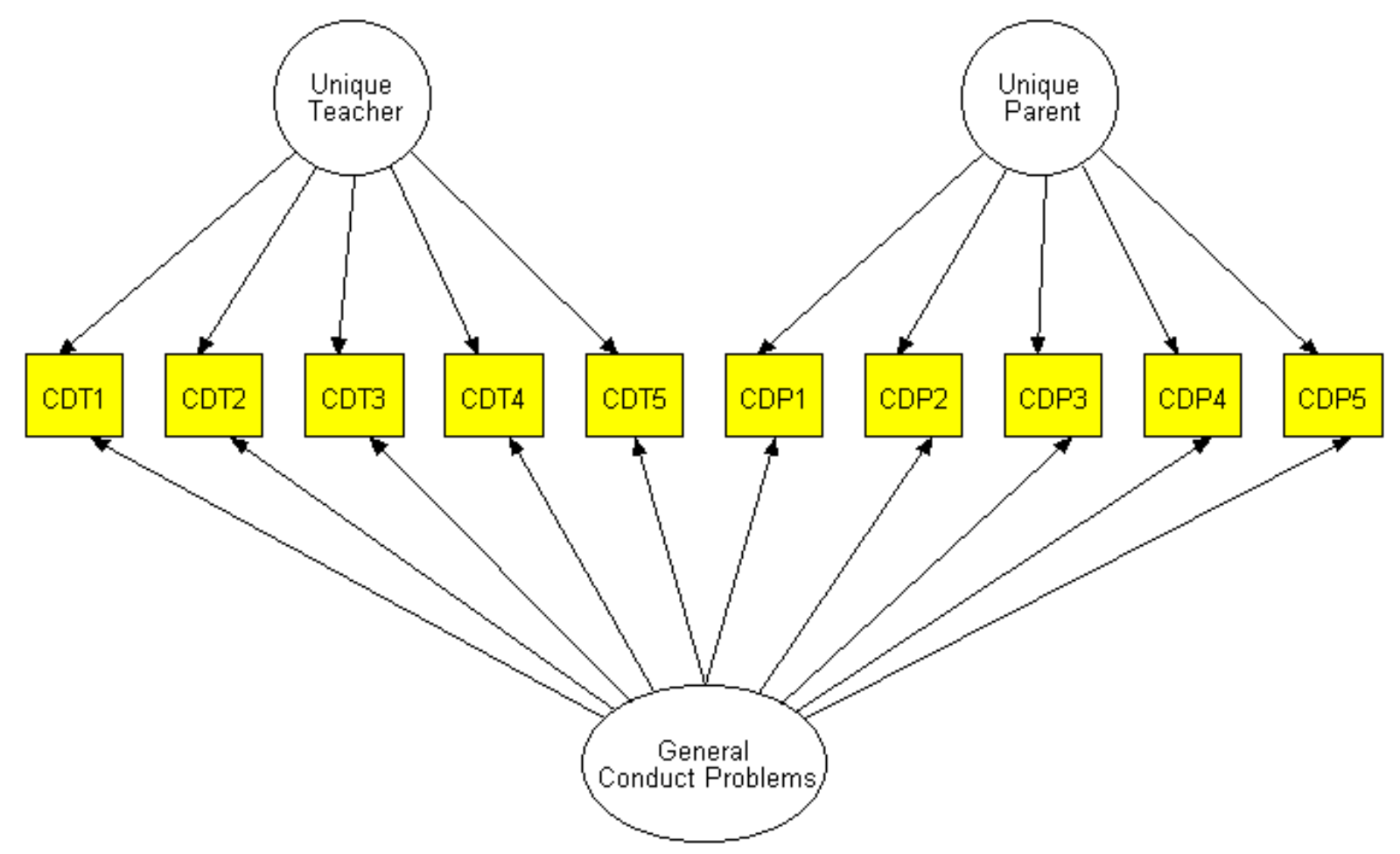

Figure 1.

The three-factor model: a general conduct problems factor and two unique informant factors. 
In our postulated model all items load on one general conduct problems factor, corresponding to the agreement between parents and teachers. In addition, the sets of parent and teacher items load on a unique parent factor and on a unique teacher factor respectively. The unique factors correspond to source effects. Measurement error is not shown in Figure 1. The reliability of parent and teacher ratings was inferred from the factor loadings on the general factor (Bollen, 1989).

The model was fitted to the covariance matrix (see Appendix) using LISREL 8 (Jöreskog \& Sörbom, 1996). Preliminary analyses revealed that the models to be tested were not invariant across gender. The model was therefore evaluated for boys and girls separately. Tests for multivariate normality led to the deletion of two cases for both boys and girls.

The model showed a good fit to the observed covariance matrices of boys $\left(\chi^{2}(25)=\right.$ $32.88, p>0.10)$ and girls $\left(\chi^{2}(25)=29.72, p>0.20\right)$. The unique factors, corresponding to source effects appeared to be significant. Deletion of the unique factors reduced the fit significantly for both boys $\left(\Delta \chi^{2}(10)=120.32, p<0.01\right)$ and girls $\left(\Delta \chi^{2}(10)=89.23, p<0.01\right)$. The results provide clear evidence of source effects in parent and teacher ratings.

Variance components. The extent to which parent and teacher ratings converge and to what extent they are affected by source effects and unreliability was calculated from the model parameters. The model parameters are displayed in Table 3 and will be discussed in more detail with respect to the visibility of conduct problems. The resulting variance components are presented in Table 2. The proportions of variance accounted for by the general conduct problems factor were generally low, ranging from $26.6 \%$ in teacher ratings of boys to $10.2 \%$ in teacher ratings of girls. The corresponding levels of agreement between teachers and parent were .45 and .35 for boys and girls respectively.

Table 2. Components of variance in teacher and parent ratings (in percentages).

\begin{tabular}{lrrrr}
\hline & \multicolumn{2}{c}{ Boys } & & \multicolumn{1}{c}{ Girls } \\
Components/Informants & Teacher & Parent & Teacher & Parent \\
\hline General conduct problems & 26.57 & 14.15 & 10.16 & 14.41 \\
Source variance & 23.57 & 11.97 & 26.49 & 9.48 \\
Random measurement error & 49.86 & 73.87 & 63.35 & 76.11 \\
\hline
\end{tabular}

The proportion of source effects differed for teachers and parents. For teachers, the proportion of source effects was approximately $25 \%$, whereas for parents it was approximately $10 \%$. This indicates that teachers are more prone to source effects than parents.

The proportions of measurement error were substantial. Given the proportion of variance accounted for by the general conduct problems and source effects, it was not surprising that the proportion of measurement error was relatively large for parents. The proportions of variance correspond to estimated reliabilities of .71 and .61 for teacher ratings 
of boys and girls respectively. The estimated reliabilities for parent ratings were .51 and .49 for boys and girls respectively.

In sum, the structural equation analyses showed that both source effects and measurement error attenuate agreement between parents and teachers. Teachers are somewhat more reliable (especially when rating boys), but also more prone to source effects than parents.

Visibility. The factor loadings of the model (Table 3) allow us to answer the question concerning the effect of the lack of visibility of more covert problem behaviors. The items CD4 (lying and cheating) and CD5 (stealing), referring to covert conduct problems, appeared to have marginal loadings on the general conduct problems factor.

For both teachers and parents, the factor loadings of the items referring to covert behavior were compared to the factor loadings of the items referring to overt behavior. Thus, six comparisons were made for each informant. For boys, all factor loadings referring to covert conduct problems appeared to be significantly smaller than the remaining loadings. The likelihood-ratio statistics of the 12 test performed ranged from $\chi^{2}(1)=4.55(p<.05)$ to $\chi^{2}(1)=51.58(p<.001)$. For girls, only 7 of the 12 tests performed were significant. Test statistics ranged from $\chi^{2}(1)=0.08$ (n.s.) to $\chi^{2}(1)=18.74(p<.01)$. The findings indicate that the lack of visibility of lying, cheating and stealing attenuates the agreement for informant ratings of boys only.

Table 3.

The three-factor model: standardized factor-loadings for boys and girls.

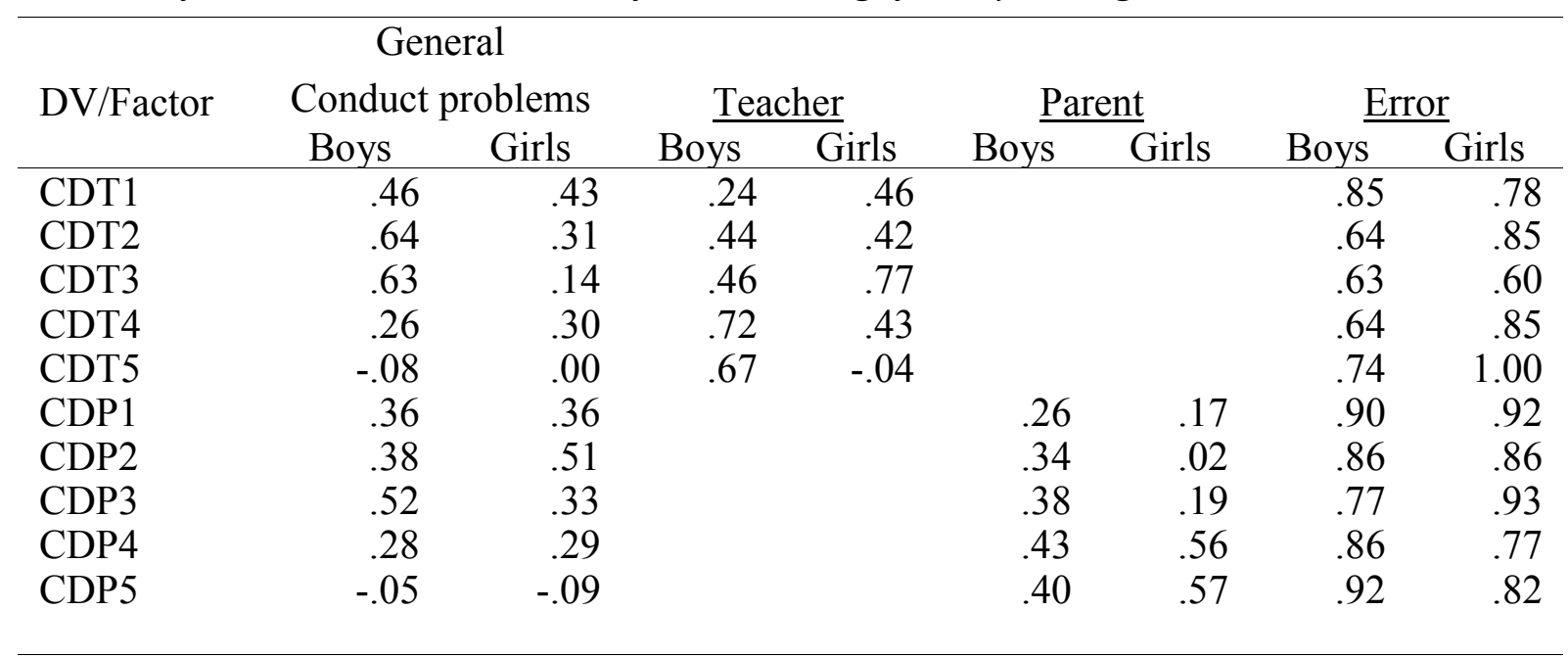

Note. $\mathrm{DV}=$ dependent variable

Explaining source effects. The question to what extent source effects in informants' judgments can be explained by situational- and informant variables was answered next. For this purpose, factor scores were computed (Bollen \& Paxton, 1998). The factor scores on the unique informant factors reflect the unique informant score of parents and teachers. The first 
six columns of Table 4 show the correlations between the unique informant scores of parent and teacher ratings and child reported oppositionality in different situations (at home, at school and with peers). The final two columns show the correlations with the teachers' norms.

The unique teacher score appeared to be significantly related to oppositionality at school for both boys and girls, and to oppositionality with peers, but only for boys. The unique teacher score was also significantly related to their norms for both boys and girls. For girls, the unique parent score appeared to be significantly related to oppositionality at school and oppositionality with peers. For boys, the unique parent score did not appear to be related to oppositionality in any of the situations.

Table 4.

Correlations between the unique informant score and situational-and informant variables.

\begin{tabular}{|c|c|c|c|c|c|c|c|c|}
\hline \multirow[b]{3}{*}{ Unique score } & \multicolumn{6}{|c|}{$\underline{\text { Situational }}$} & \multirow{2}{*}{\multicolumn{2}{|c|}{$\begin{array}{c}\text { Informant } \\
\text { Teacher norm }\end{array}$}} \\
\hline & \multicolumn{2}{|c|}{ Home } & \multicolumn{2}{|c|}{ School } & \multicolumn{2}{|c|}{$\underline{\text { Peers }}$} & & \\
\hline & Boys & Girls & Boys & Girls & Boys & Girls & Boys & Girls \\
\hline Teacher & .06 & -.01 & $.29 * *$ & $.27 * *$ & $.20 * *$ & .09 & $.22 * *$ & $.22 * *$ \\
\hline Parent & .06 & .08 & .05 & $.17 * *$ & .04 & $.21 * *$ & & \\
\hline
\end{tabular}

Note $^{* *} p<.01$

To test the combined effects of oppositionality in the three different situations and of teacher norms on the source effects, regression analyses were performed. For the unique teacher score in ratings of boys' conduct problems, this resulted in a regression equation with oppositionality at school $(\beta=.36, p<.01)$ and oppositionality at home $(\beta=-.14, p<.05)$ and teacher norms $(\beta=.15, p<.05)$ as significant predictors. The explained variance amounted to $12 \%$. For girls, the resulting regression equation contained oppositionality at school $(\beta=.33$, $p<.01)$, oppositionality at home $(\beta=-.18, p<.05)$, and teacher norms $(\beta=.18, p<.05)$ as predictors. The explained variance was $13 \%$. The results indicate that source effects in teacher ratings contain information about children who are more oppositional at school than at home, or more oppositional at home than at school. Source effects in teacher ratings also appear to be related to teacher norms with regard to conduct problems.

Source effects in teacher ratings may not only reflect situational differences in the child's behavior and differences between teachers, but could also reflect differences between school classes. This possibility was explored by relating the average unique teacher score within classes to the mean class level of oppositionality at school (as obtained by children's self-reports). The average unique teacher score and the mean class level of oppositionality at school were moderately related $(\mathrm{N}=37$; boys: $\mathrm{r}=.35, p<0.05$; girls: $\mathrm{r}=.25, p<0.1)$, indicating that source effects in teacher ratings contain information about how oppositional the school class in its entirety behaves. 
For the unique parent score in ratings of boys' conduct problems, the regression analyses revealed that source effects in ratings of boys were not related to oppositionality in any of the situations. For girls, the resulting regression equation contained oppositionality with peers $(\beta=.21, p<.01)$ as a significant predictor, explaining $4 \%$ of the total variance.

Together the results indicate that source effects in teacher ratings can be attributed to both situational differences in children's oppositionality, differences between school classes in oppositionality and teachers' norms with regard to problem behavior. Source effects in parent ratings did not appear to be the result of situational differences in children's oppositionality.

\section{Discussion}

Factors affecting agreement between parent and teacher ratings of children's conduct problems were investigated. The results showed that a structural equation model containing one general factor and two unique informant factors could adequately describe ratings of parents and teachers. The model clearly suggests that source effects negatively affect agreement between conduct problems ratings of parents and teachers. In turn, source effects in teacher ratings appeared to be affected by situational differences in children's oppositionality and the implicit norms of the teacher. Source effects in parent ratings appeared to be less well explained. Finally, our results showed that the visibility of conduct problems affects agreement.

Several authors have suggested that source effects in informants' ratings reflect situational factors, informant factors or a combination of both (Greenbaum et al., 1994; Youngstrom et al., 2000). Until now, only the role of specific informant characteristics has been substantiated empirically. Youngstrom, Loeber and Stouthamer-Loeber (2000) specifically showed that feelings of depression and stress in the mother affect agreement between informants. The role of situational specificity of the child's behavior has only been hinted at (Greenbaum et al., 1994; Rowe \& Kandel, 1997; van der Ende, 1999). In the present study, situational factors and informant factors were studied simultaneously by relying on children's self-reported oppositionality in different situations and teachers' mean rating. Following this strategy, the combined influence of situational factors and informant factors could be demonstrated.

Source effects in teacher ratings of children's conduct problems were positively related to children's oppositionality displayed at school, whereas it was negatively related to oppositionality displayed at home. This suggests that teachers are perceptive to children who, according to their self-reports, are more oppositional at school than at home or are more oppositional at home than at school. Teachers thus appear to provide unique valid situationspecific information about the child's conduct problems. 
Source effects in teacher ratings also appeared to be related to the individual teachers' mean rating of all children in their class. These mean ratings are thought to reflect teacher's internal standards and norms with regard to children's conduct problems and possibly other informant characteristics such as the length of time that the teacher has known the child (Culp, Howell, McDonald Culp, \& Blankemeyer, 2001).

Source effects in teacher ratings appeared to not only reflect differences between teachers, but also differences between school classes. The average unique teacher score and the mean class level of oppositionality at school were moderately related, indicating that source effects in teacher ratings partly reflect differences between school classes. This finding suggests that teachers provide unique information that partly reflects 'real' classroom behavior. It is common practice to use teachers' mean rating to correct for differences between teachers. Given that source effects in teacher ratings partly reflect differences in the class level of problem behaviors, filtering out the variation between teachers may imply that valid information about differences between school classes is lost.

In sum, situation-specific conduct problem behavior of the child, the mean class level of conduct problems, and teacher norms contribute to source effects in teacher ratings of children's conduct problems. That is, source effects appear to be due to both situational factors and informant factors.

With respect to the parents, the results were less conclusive. The proportion of source effects in parent ratings appeared to be small and could not be accounted for by situationspecific behavior of the child. Information about characteristics of the parents was not available. In addition, parent ratings appeared to be relatively unreliable. Future studies could benefit from using a more reliable instrument to assess conduct problems and from including parent characteristics (e.g., age, number of children, parenting style, and mental health) and teacher characteristics (e.g., amount of teaching experience and preference for structured vs. unstructured classrooms).

Agreement between parents and teachers also appeared to be affected by the visibility of specific conduct problem behaviors. Covert conduct problems were rated less reliably than overt conduct problems by both parents and teachers. Surprisingly, a significant difference in inter-informant agreement of covert- and overt conduct problems was only observed for boys. This may be explained by the finding that the variance in ratings of all conduct problems was lower for girls than for boys. Because conduct problems seem to be less prevalent among girls, it is also harder to observe a difference in the level of parent-teacher agreement for covert- and overt conduct problems.

Willoughby, Kupersmidt and Bryant (2001) focusing on reports by lead and assistant teachers also observed a lack of agreement on covert conduct problems between different informants operating within the same setting. Together with the present findings this suggests that informant agreement on conduct problems is affected by the visibility of the behavior concerned. The visibility explanation is in accordance with the generally lower convergence 
between informants' ratings of emotional versus conduct problems (Achenbach et al., 1987; Fitzmaurice, Laird, Zahner, \& Daskalakis, 1995; Rowe \& Kandel, 1997). Similar to covert conduct problems, emotional problems are harder to observe than (overt) disruptive behaviors.

The present study applied a methodological approach that simultaneously estimates the separate effects of the situational specificity of the child's behavior and informant characteristics. The method involved explaining source effects in informants' ratings on the basis of children's self-reports of situation-specific behavior and informant characteristics. Although Bollen and Paxton (1998) already discussed the possibility of investigating the determinants of source effects in subjective measures, we know of no studies that have succeeded in explaining source effects in multiple informant data. The current methodological approach provides a unique opportunity to disentangle the effects due to situational factors and informant factors.

Self-reported oppositionality at school, at home and with peers was used as a proxy of conduct problems in these situations. In our perspective, this was justified because oppositionality and conduct problems are functionally and developmentally related. Defiance of authority and noncompliance are common features of both oppositionality and conduct problems (Cole \& Zahn-Waxler, 1992) and oppositionality is thought to be a developmental precursor of conduct problems (Loeber, Green, Keenan, \& Lahey, 1995).

Although replacing conduct problems by oppositionality seems justified, oppositionality is not an optimal indicator of children's conduct problems. The use of a proxy is likely to have weakened the observed relations or to have obscured true relations. In future research, the use of a child self-report specifically designed to tap conduct problems in specific situations could lead to stronger conclusions about the main factors affecting agreement between informants.

The problem how to combine information from multiple informants, is a central issue in clinical practice and scientific research (Goldwasser \& Fitzmaurice, 2001; Offord et al., 1996). The current study provides important clues regarding how to solve this problem. The main conclusion was that source effects attenuate agreement between parents and teachers. Source effects in teacher ratings appear to partly reflect valid situation-specific information about children's conduct problems. Therefore, the notion that some behaviors are more validly reported by some informants perhaps needs to be abandoned, at least if parents and teachers and conduct problems are concerned. Instead, it seems that each informant contributes different, but valid information.

This has several implications for research and clinical practice. In research-settings, it seems one could benefit from considering methods that include informant-specific information. Structural equation models as used in this study, or multivariate linear regression models (Goldwasser \& Fitzmaurice, 2001; Kuo et al., 2000) are particularly well suited for analyzing multiple informant data, but have not been applied at large. 
Clinicians generally arrive at a diagnosis by combining the information from multiple sources according to their own implicit rules. Some clinicians may consider one type of informant as the optimal source of information on specific problems (Loeber, Green, \& Lahey, 1990). The current study suggests that clinicians should not prefer one informant to the other. When bringing together discordant information, they should however consider the reliability of the informant, the context on which the informant's judgment is based (e.g. home or school) and personal characteristics of the informant involved (e.g. mental health).

Finally, the study has implications for the perennial search for optimal informants. Because each informant has its unique value, the optimal informant possibly does not exist. Instead of trying to find the optimal informant, it seems more fruitful to gain more knowledge of how each individual informant can contribute to the assessment of children's behavior and how their different views may best be combined to obtain an integrated picture of this behavior.

Appendix.

Covariance matrix and standard deviations of parent and teacher ratings of conduct problems.

\begin{tabular}{lrrrrrrrrrr}
\hline & CDT1 & CDT2 & CDT3 & CDT4 & CDT5 & CDP1 & CDP2 & CDP3 & CDP4 & CDP5 \\
\hline CDT1 & - & .078 & .069 & .055 & -.001 & .065 & .046 & .033 & .023 & .000 \\
CDT2 & .174 & - & .058 & .047 & -.001 & .032 & .041 & .020 & .005 & -.003 \\
CDT3 & .182 & .221 & - & .046 & .000 & .004 & .000 & .019 & .016 & .002 \\
CDT4 & .073 & .137 & .132 & - & .000 & .006 & .031 & .009 & .028 & -.001 \\
CDT5 & .017 & .026 & .028 & .035 & - & -.002 & .002 & .000 & -.001 & .000 \\
CDP1 & .062 & .115 & .095 & .007 & -.003 & - & .076 & .018 & .018 & .007 \\
CDP2 & .062 & .131 & .092 & .030 & .010 & .130 & - & .027 & .035 & -.003 \\
CDP3 & .061 & .096 & .098 & .032 & -.008 & .100 & .082 & - & .025 & .003 \\
CDP4 & .037 & .045 & .049 & .027 & .003 & .061 & .058 & .062 & - & .014 \\
CDP5 & .001 & .002 & -.001 & .000 & .003 & .007 & .014 & .010 & .008 & - \\
& & & & & & & & & & \\
Boys & .672 & .632 & .607 & .435 & .177 & .766 & .618 & .477 & .393 & .154 \\
Girls & .494 & .470 & .339 & .358 & .063 & .649 & .567 & .299 & .379 & .125 \\
& & & & & & & & & & \\
\hline
\end{tabular}

Note. Lower triangle is the covariance matrix for boys; upper triangle is the covariance matrix for girls. 


\section{CHAPTER 5 \\ Rebellious teens? : \\ Examining developmental changes in oppositionality during early adolescence}

Hoffenaar, P. J., Hoeksma, J. B., \& Koot, J.M. (submitted). Rebellious teens?: Examining developmental changes in oppositionality during early adolescence. Developmental Psychology 
During the toddler years, many parents are confronted for the first time with refusals to comply, objections, stubbornness, and other forms of resistance to parental rules. In this first oppositional phase, often referred to as the "terrible twos", noncompliance typically increases (Kalb \& Loeber, 2003). Opposition is expected to peak for the second time during early adolescence. Public opinion holds that early adolescence is as a period of "storm and stress". The general public expects young adolescents to be rebellious and sees increases in opposition during early adolescence as normative. Surprisingly, this popular image of adolescence is not supported by empirical evidence (Koops \& Zuckerman, 2003; Steinberg, 2000). Although rising rates of overall parent-child conflict suggest that oppositional problems are more likely during early adolescence (Arnett, 1999), empirical studies on the developmental course of oppositionality do not provide evidence for an increase in oppositionality during this period (Bongers et al., 2004; Lahey et al., 2000; Nagin \& Tremblay, 1999).

This discrepancy can be explained in at least two possible ways. First, the general public may fail to take into account important interindividual and intraindividual differences in children's behavior. Thus, a minority of children or a limited view of the child's full behavioral repertoire (e.g., within the family-context only) may be responsible for the popular belief in the storm-and-stress view. Second, the development of oppositionality has possibly not been studied extensively enough to provide a definitive picture of the developmental course of opposition. The studies available may even have misinformed us about developmental changes in early adolescence because none of these have focused on oppositionality during this specific period.

During the early adolescent years, biological maturation and increasing social cognitive abilities bring about changes in how young adolescents perceive themselves and significant others (Paikoff \& Brooks-Gunn, 1991). Adolescents' growth in social understanding supports greater autonomy and instigates a reevaluation of hierarchically organized relationships (Youniss, 1980). Young adolescents become more willing to openly disagree with their parents and come to consider more and more issues as within their personal jurisdiction rather than within their parents' legitimate authority (Smetana, 2000). For these reasons, hierarchical power struggles are assumed to be relatively universal in early adolescence (Bugental, 2000). Given the child's developing competencies in the domain of autonomy and the child's changing conceptions of authority, it seems likely that the period of early adolescence is characterized by an increase in opposition.

However, current evidence on the developmental course of oppositionality is inconclusive. For that reason, the present longitudinal study aimed to provide a better understanding of changes in oppositionality during early adolescence by examining the common and individual developmental course of oppositionality across different situations. We collected data on six measurement occasions across the preadolescent to early adolescent period. The current study differs from previous research efforts in that we assessed 
oppositionality by means a self-report instrument specifically designed to measure the full range of children's oppositional responses across different situations, i.e. the Amsterdam Scale of Oppositionality (ASO).

\section{Individual differences}

Most people see rebelliousness and resistance to authority as characteristics of adolescents taken as a group (Arnett, 1999). Parents and teachers tend to think that increases in oppositionality are typical for early adolescents, but generally infer this knowledge from personal experience, anecdotes, or observations of only a small group of children in their direct environment. In addition, contemporary parenting advice books and popular media strengthen the popular view by warning parents for the difficulties arising in early adolescence (Steinberg, 2000).

Research efforts have generally emphasized the large individual differences in the experience of storm-and-stress (e.g. Larson \& Ham, 1993). Arnett (1999) proposed a modified "storm and stress" that takes these individual differences into account. He argued that early adolescence is not universally a time of increased rebelliousness and resistance to adult authority, but that these behaviors are more likely to occur during early adolescence than other periods of life.

Longitudinal studies by Nagin and Tremblay (1999) and Bongers et al. (2004) have shed light on individual differences in the developmental course of oppositionality. By using new data analytic methods that explicitly model the individual variation in developmental trajectories (Nagin, 1999), these studies identified different groups of children who appeared to follow common developmental trajectories. Nagin and Tremblay (1999) studied a special sample consisting of mainly White, French-speaking boys from low socioeconomic areas. After an initial assessment in kindergarten (age 6), teachers rated children's oppositionality annually between the ages of 10 and 15 years on five items from the Social Behavior Questionnaire (Tremblay et al., 1991). The results revealed two groups of boys showing either stable high or low levels of opposition. Two other groups of children showed high or moderate initial levels of opposition, but became less oppositional as they grew older. None of the trajectories appeared to increase. More recently, Bongers, Koot, van der Ende, and Verhulst (2004) examined developmental changes in oppositionality from early childhood (age 4) to late adolescence (age 18) in a large longitudinal sample including multiple cohorts. Parent ratings on children's opposition were derived from the CBCL by applying the clustering of problem behaviors as proposed by Frick et al. (1993). Six different developmental trajectories were identified. Only one out of the six trajectories was characterized by an increase in oppositionality. Furthermore, only a small group of children followed this increasing trajectory $(6 \%)$. The majority followed trajectories characterized by stable or decreasing levels of opposition. 
Taken together, these longitudinal studies indicate that there is substantial heterogeneity in developmental trajectories of oppositionality and suggest that developmental increases in oppositionality are atypical. However, it should be noted that these longitudinal studies examined changes in oppositionality across broad age ranges using longitudinal data with relatively large intervals between measurement occasions. As a result, they may be quite informative of the development of oppositionality from childhood to adolescence, but provide limited information on developmental changes during the specific period of early adolescence.

\section{Situational differences}

The previous longitudinal studies have not paid attention to possible situational differences in the development of oppositionality. Nagin and Tremblay (1999) used only teacher reports and Bongers, Koot, van der Ende, and Verhulst (2004) used only parent reports. Because they used informants that typically observe children in one particular situation, the results from their longitudinal studies may have provided a limited view of the developmental course of oppositionality across different situations. Although there is no empirical evidence indicating situational specificity in developmental trajectories of oppositionality, several empirical findings and theoretical notions lead us to expect that the development of oppositionality may vary across different contexts and situations.

First, Brehm's theory of psychological reactance predicts that individuals will oppose to threats to their freedom and that the magnitude of reactance (oppositionality) is affected by characteristics of the social agent (Brehm, 1981). Families, schools, and peer groups are characterized by different grades of elasticity or flexibility (Henker \& Whalen, 1999). Parents apply rules in a rather flexible way and adapt their behavior to their child's needs, whereas teachers are bound to the rules and constraints of the school. In addition, adolescents' relationships with parents, teachers, and peers vary in the degree of closeness, power, and permanence. Both characteristics of the situation (home and school) and the relationships involved (with parents, teachers, and peers) are likely to affect the severity and the development of oppositionality.

Second, a previous cross-sectional study revealed that oppositionality is best conceptualized as a combination of emotional and behavioral dispositions and mutually related situational influences (Hoffenaar \& Hoeksma, 2002). Situational characteristics accounted for a substantial proportion of the total variation in children's oppositionality. In addition, children (aged 9 to 13 years) appeared to be more likely to express oppositional feelings to their parents than to teachers. The apparent situational specificity in oppositionality suggests that the development of oppositionality can be properly inferred from obtaining adolescents' responses in multiple situations.

Finally, several studies on concepts related to oppositionality provide indirect evidence for diverging developmental trajectories of oppositionality in different situations. 
Keiley, Bates, Dodge, and Pettit (2000) examined developmental changes in children's externalizing and internalizing behaviors as reported by teachers and mothers. Children's externalizing problems as perceived by parents were found to decrease, whereas externalizing problems as perceived by teachers were found to increase. Similarly, Chan, Ramey, Ramey, and Schmitt (2000) examined developmental changes in parent and teacher ratings of children's social skills. Social skills as perceived by parents were found to increase, whereas social skills as perceived by teachers were found to decrease. Although the findings by Keiley et al. (2000) and Chan et al. (2000) could reflect differing expectations regarding children's behavior for parents and teachers, they give some ground for the expectation that developmental changes in oppositionality at home and at school may be quite different. Taken together, the above considerations and findings lead us to expect that the development of oppositionality at least partly depends on the situation.

\section{The present study}

In sum, the development of oppositionality during early adolescence has not been thoroughly studied. By focusing on too broad a period (from childhood to adolescence), the existing studies may have obscured changes within early adolescence. In addition, these studies neglected situational differences in the development of oppositionality. Therefore, we choose to examine developmental changes in oppositionality in different situations, i.e. situations with parents, teachers, and peers across the preadolescent to early adolescent period (10-13 years).

Children are the only "insiders" in a range of relationships (Furman \& Buhrmester, 1985 ) and therefore provide a good opportunity to obtain measures of children's behavior across a range of situations with different relationship partners. Thus, children's self-reported oppositionality, assessed half-yearly during the last three years of elementary school, was used to estimate developmental trajectories of oppositionality in different situations. In addition, we sought to reveal the extent and character of interindividual differences in intraindividual change by decomposing individual differences in the development of oppositionality into two parts: generalized oppositionality (across situations) and situationspecific oppositionality (within situations). Finally, the present study examined developmental trajectories of oppositionality for both boys and girls.

In line with Arnett's (1999) modified storm and stress view, we expected the development of oppositionality to be characterized by an average increase and large interindividual differences. In addition, we expected the developmental course of oppositionality during early adolescence to vary across situations. Because previous studies found no gender differences in developmental changes of oppositionality (Bongers et al., 2004; Lahey et al., 2000), we expected oppositionality to increase for both boys and girls. 


\section{Method}

The data for this study were collected as part of a larger longitudinal investigation of children's development in early adolescence. Data collection started in the fall of 1999 and ended in the spring of 2003. Children, their parents and teachers participated twice a year over a period of three years (the 6th, 7th, and 8th grades). Self-reports of oppositionality were collected at each measurement occasion.

\section{Participants}

To obtain a representative sample of Dutch 6th Grade pupils, a nation-wide cluster sample of schools was drawn from a register of all Dutch primary schools. Probability of inclusion for each school was inversely proportional to the mean school size per province. Schools were contacted by phone. Approximately one in two schools refused participation for reasons not related to the variables of interest. The main reasons given were: "We are too busy" and "We already participated in research some time ago". When a school refused participation, the next school in the sample register replaced this school.

Table 1.

Sample characteristics $(N=498)$

\begin{tabular}{|c|c|c|}
\hline \multicolumn{3}{|l|}{ Characteristic } \\
\hline \multicolumn{3}{|l|}{ Gender } \\
\hline Boys & \multicolumn{2}{|c|}{$48.6 \%$} \\
\hline Girls & \multicolumn{2}{|c|}{$51.4 \%$} \\
\hline \multicolumn{3}{|l|}{ Mean age at occasion 1} \\
\hline Boys (yrs.) & \multicolumn{2}{|c|}{$9.87(.46)$} \\
\hline Girls (yrs.) & \multicolumn{2}{|c|}{$9.71(.42)$} \\
\hline \multicolumn{3}{|l|}{ Family size } \\
\hline Mean number of siblings & \multicolumn{2}{|c|}{$1.62(1.02)$} \\
\hline \multicolumn{3}{|l|}{ Family composition } \\
\hline Two-parent families & \multicolumn{2}{|c|}{$91.6 \%$} \\
\hline Single-parent families & \multicolumn{2}{|c|}{$7.5 \%$} \\
\hline Other & \multicolumn{2}{|c|}{$0.9 \%$} \\
\hline Highest educational level & Mother & Father \\
\hline Vocational training & $\overline{25.5 \%}$ & $\overline{22.7 \%}$ \\
\hline Secondary school & $55.0 \%$ & $46.1 \%$ \\
\hline Higher education & $19.5 \%$ & $31.2 \%$ \\
\hline Mean weekly working hours & $12.9(12.2)$ & $40.9(9.0)$ \\
\hline Mean age (yrs.) & $38.6(5.0)$ & $41.2(4.9)$ \\
\hline
\end{tabular}


Teachers and school principals of 17 schools agreed to participate. After the first year of data collection participating schools were asked whether the next group of 6th graders would also be willing to participate in the research. Ten of the original 17 schools agreed. Signed informed parental consent statements were obtained for the majority of children within classrooms. This resulted in a representative sample of 498 Dutch 6th Grade pupils from 27 different classes in 17 different schools. The sample was created in two waves and therefore contains two groups of 6th grade children from different classes. Because MANOVA revealed no significant differences on important sample characteristics, the two cohorts were combined. Table 1 displays the sample characteristics. The sample was fairly evenly divided according to gender and demographically diverse.

\section{Amsterdam Scale for Oppositionality (ASO)}

Oppositionality was measured by using the ASO (Hoffenaar \& Hoeksma, 2002). This self-report instrument was constructed according to a facet-design (Fiske, 1971). The items were constructed by systematically sampling from the content domain of oppositionality. A response facet and a situational facet defined the content domain of oppositionality. The elements of the response facet are oppositional emotions and oppositional behaviors. The elements of the situational facet are situations with parents, teachers, and peers. The items of the ASO represent combinations of the response facet elements and the situational facet elements. Five forced-choice items represent each combination, resulting in a total of 30 items. Each item consists of a description of the situation (interactions with parents, teachers, or peers), followed by two response alternatives: one oppositional alternative and one nonoppositional response alternative. These alternatives were either emotional responses or behavioral responses.

An example of an item reflecting a behavioral response in interactions with parents is (with assigned scores between brackets): When my dad or mum tells me to help, I do it (0)/I pretend I haven't heard (1). An item reflecting oppositional feelings towards the teacher is: When the teacher punishes me, I feel sad (0)/I get angry (1). An item reflecting an emotional response in situations with peers: When another child is looking for trouble, I feel like fighting (1)/I want to walk away (0). Summing the responses within situations results in scores for oppositionality in situations with parents, teachers, and peers, ranging from 0 to 10 .

The validity of the ASO has been supported in two studies. In the first, cross sectional study of 1,158 children, we examined the structural validity of the ASO using confirmatory factor analyses (Hoffenaar \& Hoeksma, 2002). Supportive evidence was found for a structural model consisting of interrelated emotional and behavioral response factors and three mutually related situational factors. The factors representing the response tendencies and the factors representing the situational effects appeared to be independent. Because oppositionality appeared to be a combination of two independent elements, aggregation over responses within situations is justified. 
The second study investigated the value of self-reported oppositionality as obtained by the ASO (Hoffenaar, Hoeksma, and Koot, submitted). The criterion-related validity of the ASO was supported by substantial positive correlations with parent and teacher ratings of conduct problems $(\mathrm{r}=.41)$ and hyperactivity $(\mathrm{r}=.38)$ and a negative correlation with adult ratings of prosocial behavior ( $\mathrm{r}=-.36$ ). Furthermore, unique relations between oppositionality at home and at school and parent and teacher ratings indicated that scores for oppositionality in specific situations provide valid information on situational differences.

\section{Procedure}

A research assistant or the first author visited the schools twice a year (in the fall and in the spring). The ASO, a short questionnaire on children's emotional experiences, and the SDQ (Goodman, 1997) were group administered in the classroom. After a short introduction the questionnaires were distributed and completed individually by all children. Most children completed all questionnaires in less than thirty minutes. After completion, children received a freepost pre-addressed envelope containing parental questionnaires with the request to give this envelope to their parents. Children and their parents were assured confidentiality and only their ID number appeared on the questionnaires or envelopes. Teachers also received a freepost pre-addressed envelope, in which they could return completed teacher questionnaires to the first author.

\section{Statistical Analyses}

To determine the mean developmental trajectory of oppositionality across different situations and interindividual differences in intraindividual change, the current study used a multilevel model for multivariate change (Hoeksma \& Koomen, 1992; MacCallum, Kim, Malarkey, \& Kiecolt-Glaser, 1997; Plewis, 2001). The multivariate multilevel models were estimated using the IGLS algorithm implemented in Mlwin 1.10 (Rasbash et al., 2000).

The multivariate model simultaneously models the longitudinal data of children's selfreported oppositionality in three different situations (parents, teachers, and peers) by essentially combining three multilevel models for univariate change. Measures of oppositionality in different situations were nested within measurement occasions, measurement occasions were nested within children, and children were nested within school classes. To account for the fact that children within school classes are subject to the same contextual influences, separate levels for the school class and the individual child were specified (Boyle \& Willms, 2001; Snijders \& Bosker, 1999).

The multilevel model consists of a fixed and a random part. The fixed part of the multivariate multilevel model describes the mean developmental trajectories for oppositionality in situations with parents, teachers, and peers. In the present study all trajectories were found to be linear and were consequently modeled by two parameters, i.e. an intercept and a slope parameter. The origin of the age scale was taken at 10 years. Thus, the 
intercepts reflect the estimated mean levels of oppositionality at the beginning of early adolescence (initial status). The slopes represent the mean rates of change.

The random part models differences in oppositionality between school classes and the individual developmental trajectories of oppositionality within the three different situations. It also provides the information needed to estimate the extent and character of interindividual differences in intraindividual change, i.e. individual developmental differences. By using variance functions (see Appendix) derived from Goldstein (1995), the total variance between individuals was decomposed into common variance across the three different situations and unique variance within specific situations. The common variance will be labeled as variance in generalized oppositionality and the unique variance will be labeled as situation-specific variance. In line with recommendations by Biesanz, Deeb-Sossa, Papadakis, Bollen, and Curran (2004), we choose to plot the various variance components against age to determine whether change differs across situations.

\section{Missing data}

At each measurement occasion, participants were lost because of missing data. In addition, a small number of school changes (only after the first school year) caused a loss of participants. The average number of measurement occasions available was $5.30(S D=1.17)$. For $84.5 \%$ of the children, complete data on five or six occasions were available. Small percentages of children had missing data on two $(6 \%)$, three $(3.8 \%)$, or more $(5.6 \%)$ measurement occasions. Because missing data at the level of the individual child are allowed in multilevel modeling (Snijders \& Bosker, 1999), all children were retained in the longitudinal analyses.

\section{Results}

Table 2 presents the mean ASO-scores, standard deviations and scale reliabilities for boys and girls. Mean levels of oppositionality in situations with parents and teachers increased slightly with age, whereas the mean level of oppositionality in situations with peers did not appear to change much. Standard deviations (reflecting interindividual differences) appeared to increase with age for oppositionality in all three situations. Note that the mean levels and standard deviations were almost twice as large for boys as for girls.

Table 2 further presents the alphas of the oppositionality scales and their corresponding confidence intervals. At each measurement occasion, the reliability of the oppositionality scales was adequate. 
Table 2.

Means, standard deviations, and reliabilities (with corresponding 95\% confidence intervals) of the ASO at six occasions in three different situations

\begin{tabular}{|c|c|c|c|c|c|c|}
\hline \multirow[b]{2}{*}{ Occasion } & \multicolumn{2}{|c|}{$\underline{M}$} & \multicolumn{2}{|c|}{$\underline{S D}$} & \multirow[t]{2}{*}{$\underline{\alpha}$} & \multirow[t]{2}{*}{$95 \%$ C.I } \\
\hline & Boys & Girls & Boys & Girls & & \\
\hline \multicolumn{7}{|c|}{ Parents } \\
\hline 1 & 2.13 & 1.55 & 2.26 & 1.76 & .70 & $.66-.74$ \\
\hline 2 & 2.34 & 1.74 & 2.21 & 1.93 & .70 & $.66-.74$ \\
\hline 3 & 2.41 & 1.69 & 2.36 & 1.96 & .73 & $.69-.77$ \\
\hline 4 & 2.68 & 1.84 & 2.31 & 1.94 & .71 & $.68-.76$ \\
\hline 5 & 2.63 & 1.80 & 2.51 & 2.01 & .74 & $.71-.78$ \\
\hline 6 & 2.59 & 2.21 & 2.32 & 2.23 & .72 & $.68-.76$ \\
\hline \multicolumn{7}{|c|}{ Teachers } \\
\hline 1 & 2.59 & 1.08 & 2.32 & 1.40 & .76 & $.72-.79$ \\
\hline 2 & 2.76 & 1.20 & 2.41 & 1.67 & .78 & $.76-.81$ \\
\hline 3 & 2.39 & 1.15 & 2.18 & 1.54 & .76 & $.72-.79$ \\
\hline 4 & 2.83 & 1.69 & 2.50 & 1.99 & .79 & $.76-.82$ \\
\hline 5 & 2.75 & 1.39 & 2.46 & 1.86 & .80 & $.77-.83$ \\
\hline 6 & 2.88 & 1.82 & 2.41 & 2.20 & .78 & $.74-.81$ \\
\hline \multicolumn{7}{|c|}{ Peers } \\
\hline 1 & 2.60 & .97 & 2.54 & 1.25 & .78 & $.75-.81$ \\
\hline 2 & 2.67 & .98 & 2.48 & 1.44 & .80 & $.77-.82$ \\
\hline 3 & 2.75 & .87 & 2.43 & 1.35 & .79 & $.75-.82$ \\
\hline 4 & 2.94 & 1.04 & 2.65 & 1.43 & .80 & $.78-.83$ \\
\hline 5 & 2.63 & .86 & 2.49 & 1.46 & .80 & $.77-83$ \\
\hline 6 & 2.45 & .92 & 2.34 & 1.63 & .79 & $.76-.82$ \\
\hline
\end{tabular}

Because Table 2 reveals large differences between boys and girls with regard to the standard deviations of the oppositionality scores, the multivariate multilevel models were fit separately for boys and girls. The modeling procedure started with testing a model in which the development of oppositionality across different situations is described by a single mean developmental trajectory (averaged across situations) and individual variation around this mean trajectory. Next, we modeled separate mean trajectories for oppositionality in different situations with common individual variation around the average trajectory (across situations). This significantly improved the model fit (boys: $\chi^{2}(4)=20.13, p<.001$; girls: : $\chi^{2}(4)=310.91$, $p<.001$ ), indicating that developmental changes in situations with parents, teachers, and peers do not follow the same trajectory. In the third and final model, individual differences were allowed to vary across situations. Again, this resulted in a significantly better fitting model (boys: $\chi^{2}(28)=765.62, p<.001$; girls: : $\chi^{2}(28)=886.12, p<.001$ ), indicating that interindividual differences in intraindividual change partly depend on the situation. Table $3 \mathrm{a}$ and $3 \mathrm{~b}$ display the parameter estimates for the final multivariate multilevel models for boys and girls, respectively. 
Table 3a.

Boys: Fixed parameters (Top) and random parameters (Bottom) for the final model describing the development of oppositionality across situations

\begin{tabular}{|c|c|c|c|c|c|c|}
\hline Parameter & \multicolumn{2}{|c|}{ Parents } & \multicolumn{2}{|c|}{ Teachers } & \multicolumn{2}{|c|}{ Peers } \\
\hline $\begin{array}{l}\text { Intercepts } \\
\text { Slopes }\end{array}$ & \multicolumn{2}{|c|}{$2.15(.15)$} & \multicolumn{2}{|c|}{$2.52(.18)$} & \multicolumn{2}{|c|}{$2.59(.18)$} \\
\hline & \multicolumn{2}{|c|}{ Parents } & \multicolumn{2}{|c|}{ Teachers } & \multicolumn{2}{|c|}{$\underline{\text { Peers }}$} \\
\hline & Intercept & Slope & Intercept & Slope & Intercept & Slope \\
\hline \multicolumn{7}{|l|}{ Level 4 (classes) } \\
\hline Parents intercept & $.25(.15)$ & & & & & \\
\hline Teachers intercept & $.31(.16)$ & & $.49(.22)$ & & & \\
\hline Peers intercept & $.23(.14)$ & & $.33(.18)$ & & $.31(.19)$ & \\
\hline \multicolumn{7}{|l|}{ Level 3 (children) } \\
\hline Parents intercept & $2.21(.29)$ & & & & & \\
\hline Parents slope & $-.18(.12)$ & $.47(.09)$ & & & & \\
\hline Teachers intercept & $1.34(.24)$ & $-.15(.12)$ & $2.03(.30)$ & & & \\
\hline Teachers slope & $.03(.11)$ & $.28(.07)$ & $-.12(.12)$ & $.34(.08)$ & & \\
\hline Peers intercept & $1.68(.28)$ & $-.31(.14)$ & $1.91(.29)$ & $-.09(.13)$ & $3.33(.41)$ & \\
\hline Peers slope & $-.31(.12)$ & $.35(.07)$ & $-.23(.12)$ & $.30(.07)$ & $-.34(.14)$ & $.42(.09)$ \\
\hline \multicolumn{7}{|l|}{ Level 2 (residuals) } \\
\hline Parents intercept & $1.80(.09)$ & & & & & \\
\hline Teachers intercept & $.81(.07)$ & & $2.21(.11)$ & & & \\
\hline Peers intercept & $.52(.07)$ & & $.78(.08)$ & & $1.98(.10)$ & \\
\hline
\end{tabular}

Note. Standard errors are displayed in parentheses. Within-situation variances and covariances are displayed in italics.

Boys. The fixed parameters of the model (top part of Table 3a) describe the mean developmental trajectories in the three different situations as graphically depicted in Figure 1. The average trajectory corresponds to the mean trajectory across the three different situations. Figure 1 reveals that boys' overall level of oppositionality averaged across situations increased during early adolescence. Boys' initial level of oppositionality was relatively low in situations with parents (intercept $=2.15, S E=.15$ ) compared to the initial level of oppositionality in situations with teachers (intercept $=2.52, S E=.18$ ) and peers (intercept $=2.59$, $S E=.18)$. On average, boys' scores increased $.21(S E=.06)$ points per year on the scale for oppositionality in situations with parents. Their scores increased somewhat less in situations with teachers, namely .12 $(S E=.06)$ points per year. Boys' scores for oppositionality in situations with peers did not appear to change (slope $=.00, S E=.06$ ). These findings indicate an overall increase in boys' oppositionality and suggest that the specific nature of change depends on the situation. 
Figure 1.

Mean developmental trajectories of oppositionality in different situations for boys and girls.
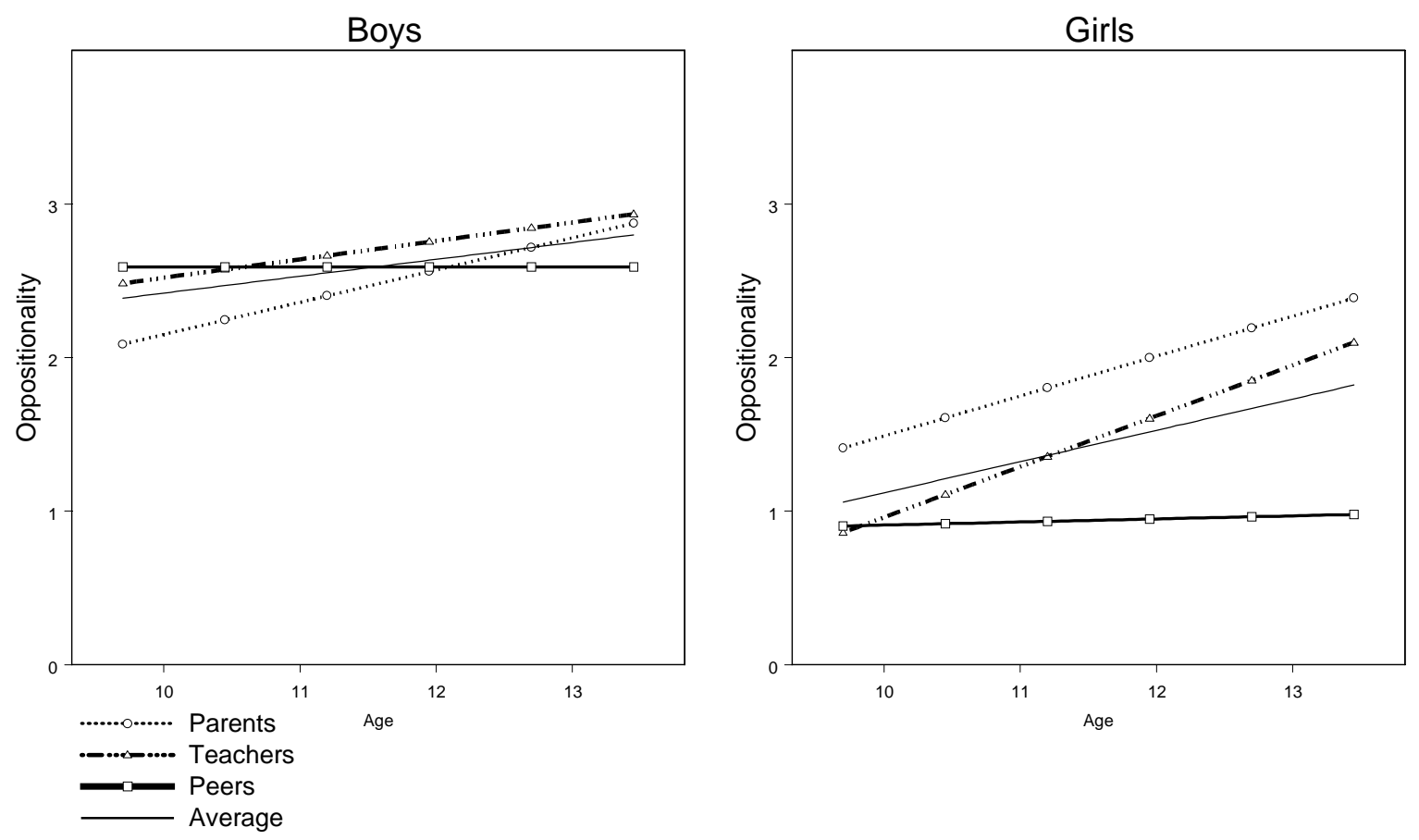

Next, we derived estimated individual developmental trajectories of the overall level of oppositionality averaged across situations from the random part of the model (bottom part of Table $3 b$ ). Figure 2 a depicts a random selection of $10 \%$ of the individual trajectories. They reveal considerable heterogeneity in the development of oppositionality. Most of the trajectories show an increase in oppositionality, some trajectories show a decrease in the level of oppositionality with age and a relatively constant level of oppositionality characterizes others. Overall, the pattern in the individual developmental trajectories appears to be a diverging fan-spread, indicating increasing individual variability in the level of oppositionality.

Next, we used the parameter estimates in table $3 \mathrm{a}$ to compute the common developmental variance and the unique developmental variance in different situations. Results are depicted in Figure $2 \mathrm{~b}$. In line with the observed pattern in the individual developmental trajectories, Figure $2 \mathrm{~b}$ reveals that the variance between individuals due to systematic change increased with age, pointing to increasing divergence. The developmental variance was decomposed into interindividual differences in generalized oppositionality and interindividual differences in situation-specific oppositionality. Approximately half of the developmental variance (range from $56 \%$ to $58 \%$ ) is variance in generalized oppositionality. The remaining developmental variance is situation-specific variance. The three situation-specific variance components appeared to be of similar size, with average proportions of $14 \%, 12 \%$, and $17 \%$ for situations with parents, teachers, and peers, respectively. The proportion of situationspecific developmental variance indicates that a substantial part of the interindividual 
Chapter 5

differences in intraindividual change can be attributed to situations, suggesting that the process of oppositionality development is partly dependent on the situation in which it occurs.

Figure $2 \mathrm{a}$ and $2 \mathrm{~b}$.

Boys: Development of oppositionality

Random sample (10\%) of the individual developmental trajectories

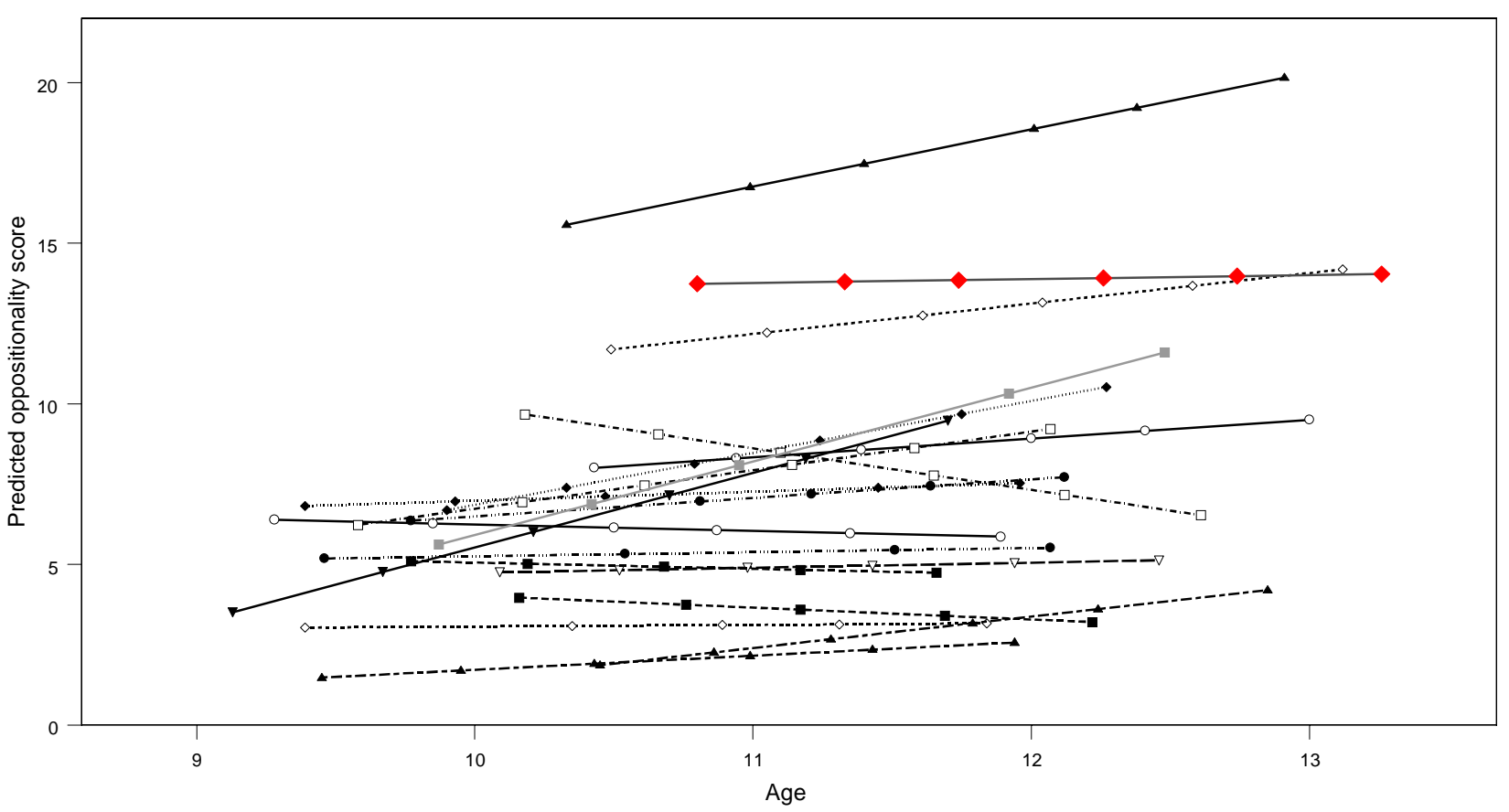

Boys

Developmental variance across age

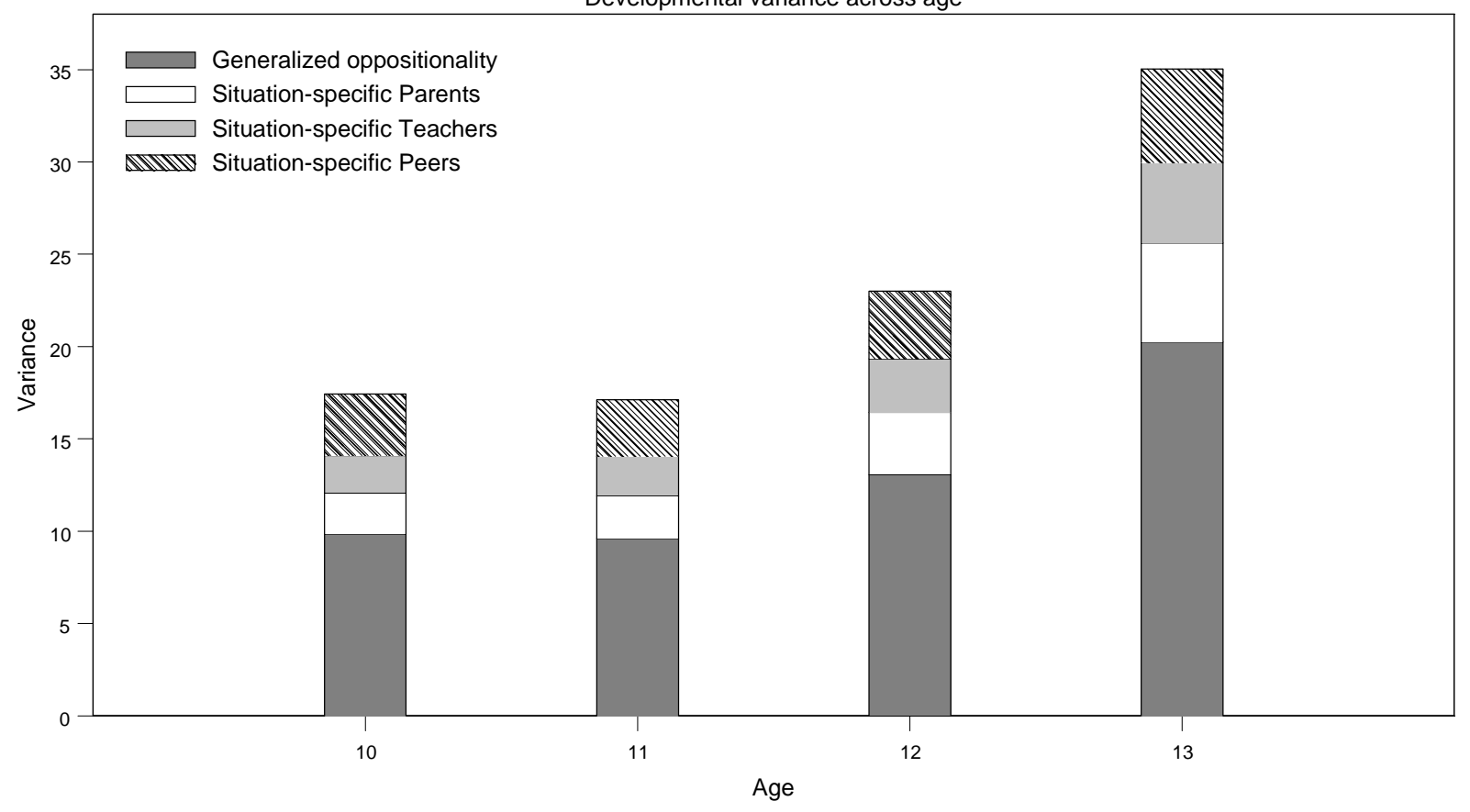


Girls. The same analyses were repeated for girls. The fixed part of the model (top part of Table 3b) describes the mean trajectories for girls' oppositionality in different situations. The average trajectory across situations as well as the mean trajectories in situations with parents, teachers, and peers is depicted in Figure 1. The average trajectory increased, indicating that girls' overall level of oppositionality increased during the early adolescent years. Initially, girls appear to be somewhat more oppositional in situations with parents (intercept $=1.49, S E=.14$ ) than in situations with teachers (intercept=.96, $S E=.11$ ) and peers (intercept=.91, $S E=.09)$. Girls' scores increased $.26(S E=.09)$ on the scale for oppositionality in situations with parents per year and $.33(S E=.06)$ per year on the scale for oppositionality in situations with teachers. The level of oppositionality in situations with peers remained constant across age (slope $=.02, S E=.04$ ). These findings indicate an overall increase in girls' oppositionality and suggest that intraindividual change in oppositionality in situations with peers follows a different trajectory.

Table 3b.

Girls: Fixed parameters (Top) and random parameters (Bottom) for the final model describing the development of oppositionality across situations

\begin{tabular}{|c|c|c|c|c|c|c|}
\hline Parameter & \multicolumn{2}{|c|}{ Parents } & \multicolumn{2}{|c|}{ Teachers } & \multicolumn{2}{|c|}{ Peers } \\
\hline $\begin{array}{l}\text { Intercepts } \\
\text { Slopes }\end{array}$ & \multicolumn{2}{|c|}{$1.49(.14)$} & \multicolumn{2}{|c|}{$.96(.11)$} & $.91(.09)$ & 4) \\
\hline & \multicolumn{2}{|c|}{ Parents } & \multicolumn{2}{|c|}{ Teachers } & \multicolumn{2}{|c|}{ Peers } \\
\hline & Intercept & Slope & Intercept & Slope & Intercept & Slope \\
\hline \multicolumn{7}{|l|}{ Level 4 (classes) } \\
\hline Parents intercept & $.22(.12)$ & & & & & \\
\hline Teachers intercept & $.07(.08)$ & & $.15(.08)$ & & & \\
\hline Peers intercept & $.08(.06)$ & & $.02(.04)$ & & $.08(.05)$ & \\
\hline \multicolumn{7}{|l|}{ Level 3 (children) } \\
\hline Parents intercept & $1.91(.25)$ & & & & & \\
\hline Parents slope & $-.29(.11)$ & $.44(.07)$ & & & & \\
\hline Teachers intercept & $1.05(.16)$ & $-.29(.08)$ & $.95(.15)$ & & & \\
\hline Teachers slope & $-.12(.10)$ & $.31(.06)$ & $-.15(.08)$ & $.46(.07)$ & & \\
\hline Peers intercept & $.70(.14)$ & $-.20(.07)$ & $.50(.11)$ & $-.13(.07)$ & $.86(.12)$ & \\
\hline Peers slope & $-.24(.08)$ & $.24(.04)$ & $-.17(.06)$ & $.24(.04)$ & $-.21(.06)$ & $.26(.04)$ \\
\hline \multicolumn{7}{|l|}{ Level 2 (residuals) } \\
\hline Parents intercept & $1.45(.07)$ & & & & & \\
\hline Teachers intercept & $.46(.05)$ & & $1.24(.06)$ & & & \\
\hline Peers intercept & $.25(.04)$ & & $.29(.04)$ & & $.86(.04)$ & \\
\hline
\end{tabular}

Note. Standard errors are displayed in parentheses. Within-situation variances and covariances are displayed in italics. 
From the random part of the model (bottom part of Table 3b), we derived estimated individual developmental trajectories of the overall level of oppositionality averaged across situations. The random selection of $10 \%$ of the trajectories displayed in Figure 3 a reveals considerable heterogeneity in the development of girls' oppositionality. Most of the trajectories appear to be characterized by increasing or constant levels of oppositionality, but the selection of trajectories also contains a decreasing trajectory. Again, the overall pattern suggests that the level of oppositionality "fans out", i.e. interindividual differences in developmental trajectories increase with age.

The estimates of the variance-components displayed in Figure $3 \mathrm{~b}$ and the observed diverging fan-spread pattern agree: the variance between individuals due to systematic change increased with age. Approximately half of the total developmental variance between individuals (range from $50 \%$ to $53 \%$ ) is variance in generalized oppositionality and the other half is made up of three situation-specific variance components varying somewhat in size. At age 10 , the proportion of situation-specific developmental variance in situations with parents is larger $(24 \%)$ than the same proportion in situations with teachers $(12 \%)$ and peers $(10 \%)$. At age 13, the proportions of situation-specific developmental variance in situations with parents and teachers are more or less equal $(20 \%)$ and both greater than the proportion of variance specific to situations with peers $(10 \%)$. The results reveal considerable situational differences in interindividual differences in intraindividual change, suggesting situational dependence in the development of girls' oppositionality.

Boys and girls. The apparent differences in the developmental patterns of oppositionality between boys and girls raise the question if and how differences between the two sexes in oppositionality changed over the course of development. To address this issue, we first used the estimated fixed parameters of the multilevel models (Table $3 a$ and $3 b$ ) to predict the mean levels of oppositionality across age. Comparison of the age-specific estimates revealed that the mean level of oppositionality for boys was higher than for girls at all ages. However, the difference in the estimated mean level of boys' and girls' oppositionality declined from 1.56 at age 10 to .93 at age 13 . Next, we used the model to predict individual levels of oppositionality. Using the estimated individual levels at ages 10, 11,12 , and 13, it was found that the percentage of boys with higher scores than the most oppositional girl decreased from $11 \%$ at age 10 to $1 \%$ at age 13 . These findings indicate that girls became more similar to boys, but that they did not reach the same level of oppositionality. 
Figure $3 a$ and $3 b$.

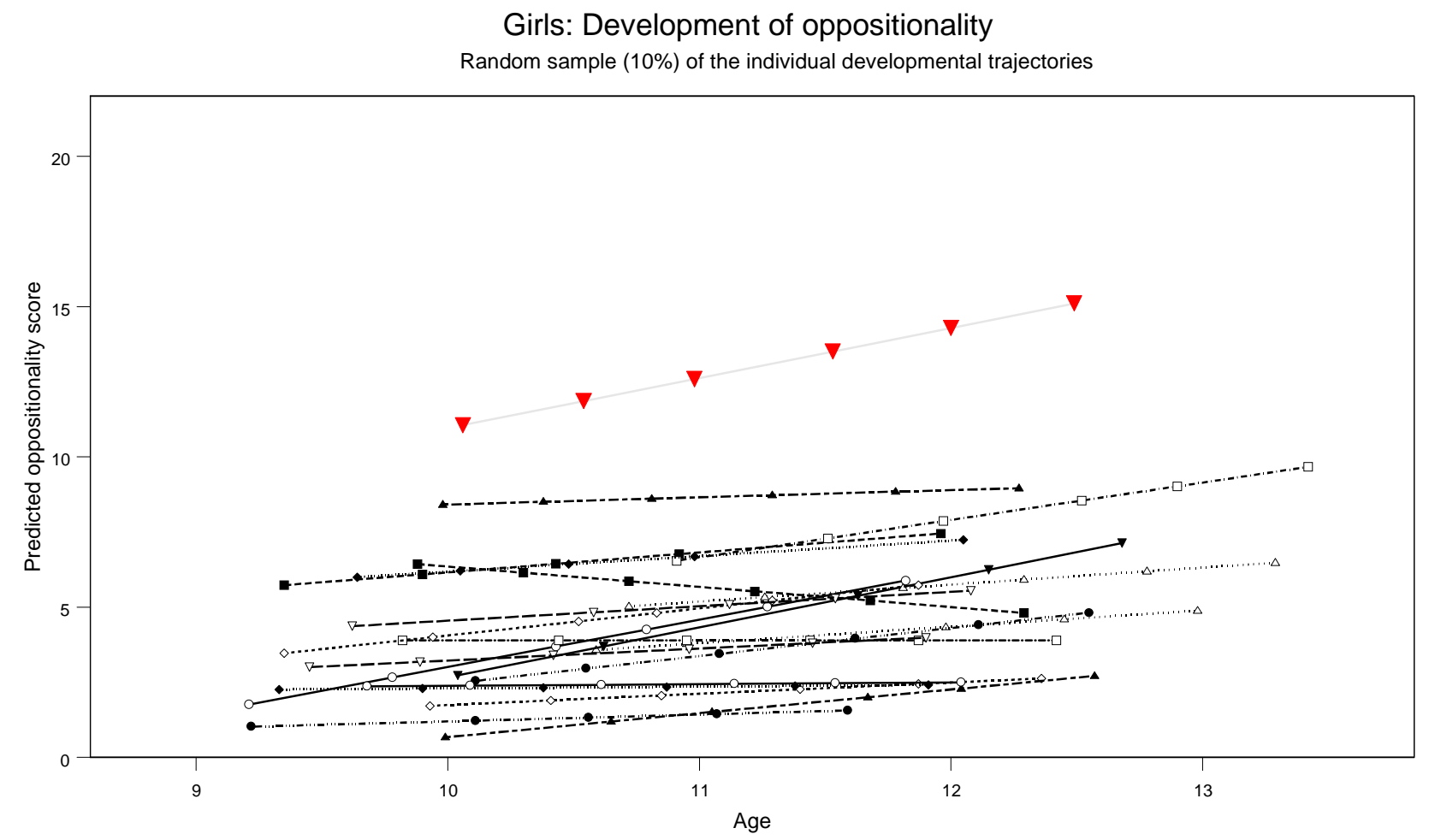

Girls

Developmental variance across age

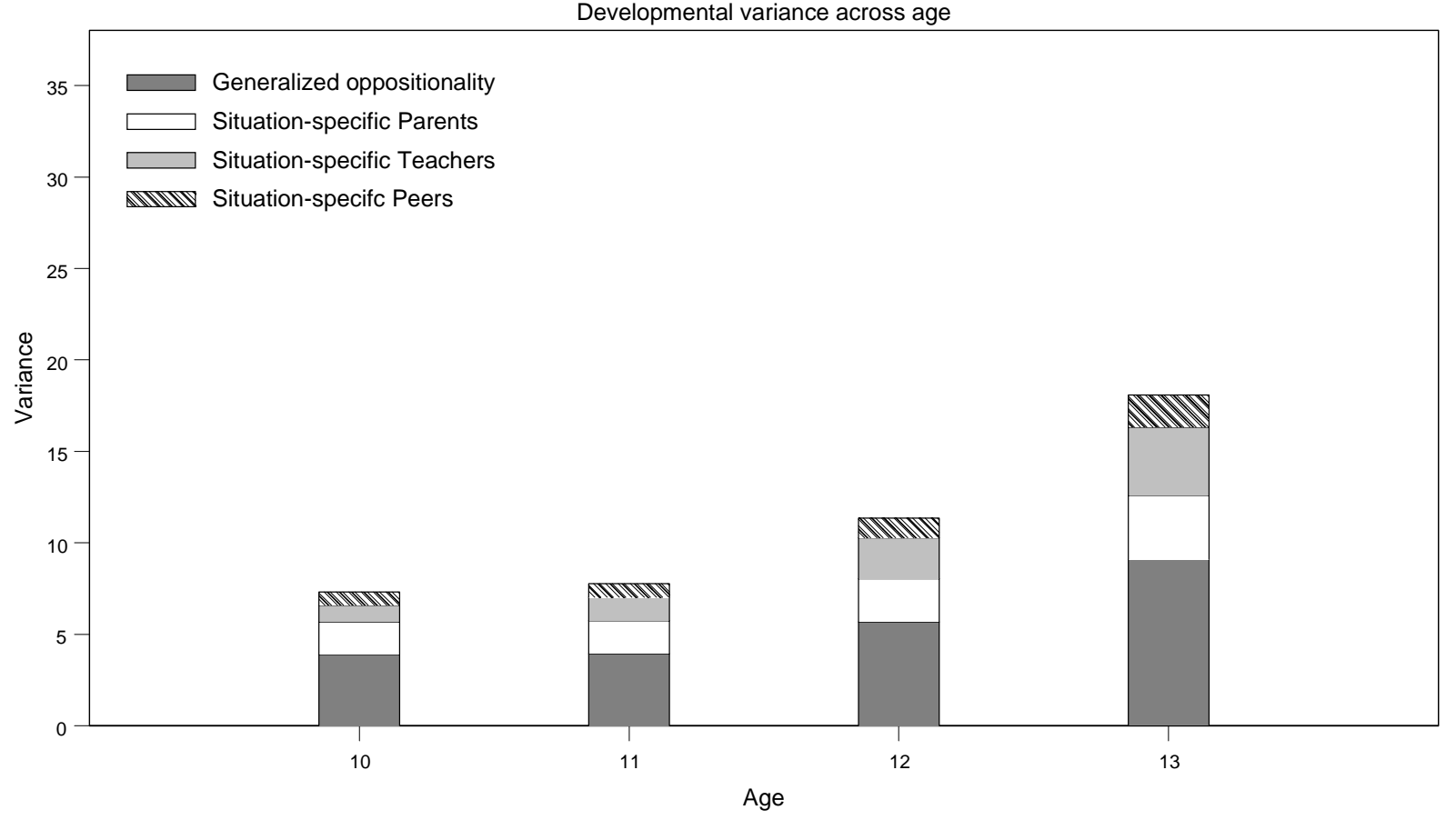




\section{Discussion}

The present study examined developmental changes in oppositionality during early adolescence. Three main findings emerged. First, both boys and girls, on average, became more oppositional during early adolescence. This upward trend was evident in children's selfreported oppositionality in situations with parents and teachers, but not in situations with peers. Second, the developmental pattern appeared to be characterized by increasing divergence between individuals with regard to the level of oppositionality. And finally, interindividual differences in intraindividual change appeared to be partly situation-specific. These findings will be discussed in the following sections.

\section{Developmental course of oppositionality}

Children typically show an increase in opposition during the last three years of elementary school. The observed developmental trend is in line with the general public's perception that early adolescence is a period in which young adolescents become more oppositional and thus increasingly frustrate their parents and teachers with their resistant and defiant behavior. The finding that oppositionality tends to increase during early adolescence also converges with research showing that, on average, the level of externalizing problems (Galambos, Barker, \& Almeida, 2003; Wångby, Bergman, \& Magnusson, 1999) and the frequency of parent-child conflicts (Arnett, 1999) increase during this period.

The findings contrast with the overall decrease in oppositionality observed in longitudinal studies over longer periods of time by Bongers, Koot, van der Ende, and Verhulst (2004) and Nagin and Tremblay (1999). Unfortunately, methodological variations hinder a direct comparison of findings across these studies. First, the nature of the sample varied. The present study was based on a representative population sample of children living in the Netherlands, the study by Bongers et al. was based on a large community sample of both boys and girls living in one particular province of the Netherlands, and Nagin and Tremblay used a sample consisting of White, French-speaking boys of schools in low socioeconomic areas in Canada. Second, the present study provides rather unique information on developmental changes in oppositionality by using child self-reports instead of parent or teacher reports. And finally, the previous longitudinal studies provided information on the development of oppositionality across broad age ranges, whereas the present study focused on early adolescence.

Given these methodological variations, we would suggest that each of these studies provides different information on the developmental course of oppositionality. By examining developmental changes in self-reported oppositionality during early adolescence, we have found preliminary evidence for an increase in oppositionality during this specific period. The other two longitudinal studies provide information on long-term developmental changes as observed by parents and teachers. The discrepancy in the observed direction of change 
suggests that the rate of change of oppositionality may not be consistent over the course of development. Therefore, it seems that research focusing on those periods in which change is most likely to occur can enrich information on developmental changes across broad age ranges (c.f. Boyle \& Willms, 2001).

\section{Interindividual differences}

There appeared to be considerable heterogeneity in the developmental trajectories of oppositionality. Some children become more oppositional, others become less oppositional, and other children appear to be characterized by either stable high or low levels of oppositionality. The notion that early adolescence is universally a time of increasing opposition should therefore be rejected. The observed increase in oppositionality together with the apparent interindividual differences in the trajectories of oppositionality supports Arnett's (1999) proposal for a modified 'storm and stress' view. Increases in oppositionality during early adolescence are not inevitable and universal, but it is recognized that many young adolescents become more willing to disagree with those in authority and that many parents and teachers perceive of early adolescence as a difficult time because of frequent (authority) conflicts.

The present study extends the previously reported heterogeneity in the development of oppositionality (Bongers et al., 2004; Nagin \& Tremblay, 1999) by providing information on how changes in oppositionality vary across individuals. This information could be revealed by considering the total range of possible changes in oppositionality, i.e. treating the individual variation in developmental trajectories as continuous (Hertzog \& Nesselroade, 2003). The socalled fan-spread pattern in the individual developmental trajectories (c.f. Rudinger \& Reitz, 1998 ) indicated that the development of oppositionality is characterized by increasing divergence between individuals. Several authors have proposed general developmental processes that may lead to increasing divergence. Cole et al. (2002) linked increasing divergence in children's depressive symptomatology to a process of differentiation, i.e. preexisting individual differences are magnified over the course of development. Graber and Brooks-Gunn (1996) describe how transitional periods can lead to increasing divergence by referring to the accentuation principle. The accentuation principle hypothesizes that behavioral patterns become more extreme at times of challenge, because individuals respond to these challenges in accordance with their existing behavioral and coping repertoire. Early adolescence is widely recognized as a developmental transition period during which obtaining a degree of autonomy represents an important developmental challenge (Paikoff \& BrooksGunn, 1991). The observed divergence in oppositionality during this period can thus be interpreted as a process of accentuation and differentiation. 


\section{Situational differences}

The direction of change appeared to depend on the situation. For both boys and girls, oppositionality typically increased in situations with parents and teachers, but not in situations with peers. Children's relationships with parents and teachers are typically authority-based relationships, whereas peers are not seen as authorities but as equals (Bugental, 2000; Reis, Collins, \& Berscheid, 2000). Therefore, our results seem to suggest that the development of oppositionality in different situations is affected by the degree to which the relationship involved is characterized by an imbalance in power. The observed developmental pattern may be related to mechanisms of relationship change (Collins, 1997). At a point in time that teenage children are becoming more critical towards adult authority and expect more behavioral freedom, parents and teachers alike may resist relinquishing their position of authority and fail to grant age-appropriate degrees of autonomy. If they perceive the child's resistance as a behavioral deviation or as a disruption in the relationship, they may even turn to more excessive forms of control. The young adolescent, in turn, may react with increased opposition. Because such cycles of opposition and negative responses from the environment (e.g. coercion) are most likely to occur in interactions with parents and teachers, they may explain the observed developmental pattern.

By decomposing the total interindividual differences in the development of oppositionality into generalized oppositionality and situation-specific oppositionality, we sought to reveal the character of the development of oppositionality. Our results clearly showed that almost half of the variance in individual developmental trajectories is situationspecific, indicating that a substantial part of the individual development of oppositionality could be attributed to situations.

Thus, the present study found situational differences in the direction of change and in interindividual differences in intraindividual change. The findings provide evidence for the situational specificity of the development of oppositionality. The process of oppositionality development appears to depend on the situation in which it occurs, thereby supporting the role of the social context in children's development (Boyce et al., 1998). On the basis of our results, we argue that the development of oppositionality can be seen as the combined effect of an unfolding maturational pattern and the adolescent's interactions with parents, teachers, and peers. Such a conceptualization of the development of oppositionality is in line with a transactional model of oppositionality (Greene \& Doyle, 1999), but contrasts with the implicit assumption in much of the previous work on oppositionality and the broader category of externalizing behaviors that the development of these behaviors can be regarded as a withinindividual process (Munson, McMahon, \& Spieker, 2001).

Previous studies have used both parent and teacher reports to study the development of children's behavior and social skills across different situations (Chan et al., 2000; Keiley et al., 2000). The findings suggest that children's development varies across situations, but the observed difference in trajectories based on different informants can also be ascribed to 
differences in the perceptions of informants (cf. Achenbach, McConaughy et al. 1987). Because we relied on the information of the same 'observers' across different situations, i.e. children themselves, the situation in which the behavior is exhibited and the data source were not confounded in the present study. The results strengthen the conclusions from previous research that very probably real differences exist in the child's development across different situations. These differences are likely to underlie the lack of concordance in the trajectories of parent-reported and teacher-reported behaviors.

\section{Gender differences}

Comparing the trajectories of boys and girls revealed two striking gender differences. First, oppositionality appeared to increase at a more rapid rate for girls than for boys. The different paces resulted in a gradually decreasing gender difference in the mean level of oppositionality. Most noteworthy, the proportion of boys whose level of oppositionality was higher than that of the most oppositional girl declined substantially. By the end of early adolescence, it appears that the extreme cases among boys and girls do not longer differ from one another. The results strongly suggest that girls catch up with boys in the level of oppositionality during early adolescence. This provides additional support for the hypothesis that gender differences in conduct problems become less pronounced over the course of development as a result of steeper increases in conduct problems from childhood to adolescence for girls than for boys (Silverthorn \& Frick, 1999).

Second, the faster rate of change in girls' oppositionality appeared to be most evident in situations with teachers. The developmental increase of girls' oppositionality at school stood out in comparison to their increase in oppositionality at home and the observed developmental increase in boys' oppositionality. This can possibly be explained by the fact that girls generally begin puberty earlier than boys. As a result, teachers may face a classroom with substantial gender differences in psychological and physical maturity. Although these differences may call for a differential treatment of boys and girls, teachers may generally apply the rules to all children in the same way. As a result, girls may be more likely than boys to feel undeservedly treated as immature by their teacher. These feelings may stimulate the development of girls' oppositionality within the school context.

\section{Future research}

Several potential directions for future research can be given. First, our use of six measurement waves to study developmental changes across a three-year period was more extensive than in most developmental studies. Nevertheless, future research efforts could include extra measurement occasions at later ages. Such studies could test the assumption of a peak in the level of oppositionality during early adolescence (Kalb \& Loeber, 2003) by examining whether the observed increase in oppositionality is followed by a decrease in midadolescence. In addition, such studies would provide developmental data on the period in 
which boys are most likely to experience pubertal changes. Given the supposed impact of puberty on the development of oppositionality, it could be that boys' oppositionality increases at a faster rate during middle adolescence. Second, large differences between cultures in the experience of adolescence (Arnett, 1999; Koops \& Zuckerman, 2003) indicate the necessity to replicate this study with samples from other countries. And finally, replication of the current findings with information from other data sources is very welcome. However, such replications are complicated by the fact that few methods are available to obtain situationspecific information from adult informants and the high costs and relative inefficiency of observations in multiple situations at multiple occasions.

\section{Final comments and implications}

The general public often thinks that a young adolescent equals a rebellious teenager. In line with the conclusion of Arnett (1999) and Steinberg (2000) that the general public's conception of adolescence is not entirely wrong, the present study indicates that many young teens really do become more oppositional during early adolescence. Nevertheless, the popular belief on the developmental course of oppositionality leaves little room for differences between individuals and differences in the situations in which children display their oppositionality. One could therefore argue that despite its accuracy, the popular view of early adolescence is still somewhat of a stereotype.

The findings have implications for the assessment of clinically relevant manifestations of oppositionality. The increased understanding of the developmental course of oppositionality during early adolescence can help to facilitate the creation of a developmentally sensitive classification system (Willoughby, 2003). The DSM-IV criteria for Oppositional Defiant Disorder (ODD; APA, 1994) include the requirement that its symptoms must be evident for at least 6 months, but do not include other temporal or developmental requirements. The importance of considering the developmental course of a child's symptomatology is becoming increasingly appreciated, with some authors even arguing for a shift in new editions of the DSM toward a more dimensional model of classification, which includes the continuum of the child's functioning across time (Widiger \& Clark, 2000). The current study demonstrates considerably variation in oppositionality across individuals, time, and situations. We would therefore argue that the construction of a DSM-V category of ODD could benefit from considering the developmental course of oppositionality during early adolescence as well as the situational specificity of oppositionality. 


\section{Appendix.}

The next table represents the variance covariance matrix of the random parameters at level 2 (between individuals) in the multivariate multilevel model. It is used to compute the variance in generalized oppositionality and situation-specific variance.

\begin{tabular}{|c|c|c|c|c|}
\hline \multicolumn{2}{|c|}{ Parents } & \multicolumn{2}{|c|}{ Teachers } & Peers \\
\hline Intercept & Slope & Intercept & Slope & Intercept \\
\hline
\end{tabular}

Parents intercept $\left(ß_{10}\right)$

Parents slope $\left(\beta_{11}\right)$

Teachers intercept $\left(\beta_{20}\right)$

Teachers slope $\left(\beta_{21}\right)$

Peers intercept $\left(\beta_{30}\right)$

Peers slope $\left(\beta_{31}\right)$

$$
\sigma_{10}^{2}
$$

$\sigma_{10,11}$

$\sigma_{10,20}$

$\sigma_{10,21}$

$\sigma_{10,30}$

$\sigma_{10,31}$

$\sigma_{20}^{2}$
$\sigma_{20,21}$
$\sigma_{20,30}$
$\sigma_{20,31}$

$$
\sigma_{11}^{2}
$$$$
\sigma_{11,20}
$$$$
\sigma_{11,21}
$$$$
\sigma_{11,30}
$$$$
\sigma_{11,31}
$$

$$
\begin{gathered}
\sigma_{21}{ }^{2} \\
\sigma_{21,30} \\
\sigma_{21,31}
\end{gathered}
$$$$
\begin{gathered}
\sigma_{30}^{2} \\
\sigma_{30,31}
\end{gathered}
$$$$
\sigma_{31}^{2}
$$

To illustrate, $\sigma_{10}{ }^{2}$ refers to the variance of the intercept of oppositionality in situations with parents, $\sigma_{10,11}$ refers to the covariance between the intercept and the rate of change of oppositionality in situations with parents, etc.

The unique variance between individuals in specific situations (situation-specific variance) is computed according to:

$\operatorname{Var}\left(\right.$ Parents $_{i j} \mid$ Age $\left._{i j}\right)=\sigma_{10}^{2}+2 * \sigma_{10,11}$ Age $_{i j}+\sigma_{11}^{2}$ Age $_{i j}^{2}$,

$\operatorname{Var}\left(\right.$ Teachers $\left._{i j} \mid \operatorname{Age}_{i j}\right)=\sigma_{20}^{2}+2 * \sigma_{20,21} \operatorname{Age}_{i j}+\sigma_{21}^{2} \operatorname{Age}_{i j}^{2}$,

$\operatorname{Var}\left(\right.$ Peers $\left._{i j} \mid A g e_{i j}\right)=\sigma_{30}{ }^{2}+2 * \sigma_{30,31} A_{g e_{i j}}+\sigma_{31}{ }^{2} \operatorname{Age}^{2}{ }_{i j}$,

, where $A g e_{i j}$ is person $j$ 's age at time $i$.

The common variance across the three situations (generalized oppositionality) is computed as follows

$$
\begin{aligned}
\operatorname{Var}\left(\text { Generalized }_{i j} \mid \text { Age }_{i j}\right) & =2^{*}\left(\sigma_{10,20}+\sigma_{10,21} \text { Age }_{i j}+\sigma_{11,20} A g e_{i j}+\sigma_{11,21} A g e^{2}{ }_{i j}\right) \\
& +2^{*}\left(\sigma_{10,30}+\sigma_{10,31} \text { Age }_{i j}+\sigma_{11,30} \text { Age }_{i j}+\sigma_{11,31} A g e^{2}{ }_{i j}\right) \\
& +2 *\left(\sigma_{20,30}+\sigma_{20,31} A g e_{i j}+\sigma_{21,30} A g e_{i j}+\sigma_{21,31} A g e^{2}{ }_{i j}\right) .
\end{aligned}
$$




\section{CHAPTER 6}

\section{The developmental significance of oppositionality during early adolescence: Trajectories toward academic, social, and psychological adjustment}

Hoffenaar, P. J., Hoeksma, J. B., \& Koot, J.M. (in preparation). The developmental significance of oppositionality during early adolescence: Trajectories toward academic, social, and psychological adjustment. 
Oppositionality consists of a wide range of behaviors that can occur in a variety of situations. Temper outbursts, stubborn and negative behavior, disobedience, and hostility have all been noted as behaviors related to oppositionality (Gard \& Berry, 1987). These behaviors are usually observed in situations with parents and teachers, but can also occur in situations with other persons with whom the child is familiar, such as peers (Greene \& Doyle, 1999). Resistance to adult directions and unwillingness to negotiate with peers have in common that they reflect the child's refusal to conform to social rules. The core element of oppositionality appears to be a willful contrariness; the child does not do what he or she is supposed to be doing (Redl, 1976).

The developmental significance of oppositionality during early adolescence is largely unknown. Some have suggested that oppositionality is the starting point from which the child progresses toward more serious forms of problem behavior (Loeber et al., 1993). Others argue that oppositionality in young adolescents is not a precursor to antisocial behaviors, but an indication of developing competencies in the domain of autonomy and self-definition (Baumrind, 1997; Mones, 1998). The present study aimed to clarify the developmental significance of oppositionality during early adolescence by examining the link between developmental patterns across the preadolescent to early adolescent period and a comprehensive set of developmental outcomes. To examine the role of gender in the developmental significance of oppositionality, the present study included both boys and girls.

Few studies have considered the development of oppositionality. The identification of individual differences in the development of oppositionality requires longitudinal studies (Baltes \& Nesselroade, 1979; Wohlwill, 1973). In turn, longitudinal studies require concepts and instruments that are sensitive to change. The diagnostic category of oppositional defiant disorder (ODD) and its diagnostic criteria may be less useful in this respect. ODD characterizes those children who display a recurrent pattern of developmentally inappropriate levels of opposition manifested by negativistic, hostile and deviant behavior (American Psychiatric Association, 1994). This categorical approach to the assessment of oppositionality only differentiates between normal and deviant behavior and may therefore not be suited to study deviations over time away from normative developmental patterns (Sroufe, 1997). In addition, a categorical approach obscures more subtle inter- and intraindividual differences in oppositionality. Instruments measuring the full range of oppositional responses may more easily reveal developmental changes in oppositionality. For that reason, Hoffenaar and Hoeksma (2002) developed the Amsterdam Scale of Oppositionality (ASO). This instrument consists of items tapping children's self-reports of emotions and behaviors during normal everyday interactions with parents, teachers, and peers and offers a continuum of oppositional responses ranging from normal resistance to external influences to more disruptive actions, such as temper tantrums, extreme disobedience and negativistic, hostile behaviors (c.f. Gard \& Berry, 1987). 
Clarification of the developmental significance of oppositionality requires an understanding of how the development of oppositionality is related to both positive and negative outcomes. A certain degree of defiance and noncompliance may well be acceptable to parents and teachers. Nevertheless, repeated failure to fulfill ordinary requirements of obedience may have severe consequences in the long run if the child's opposition increases and accumulates. Despite the strong emphasis on the developmental process in recent models of individual development (Bergman, Magnusson, \& El Khouri, 2003), only a few studies have examined developmental trajectories linking change in early behaviors to later developmental outcomes. As shown in studies on the development of children's aggression (Schaeffer, Petras, Ialongo, Poduska, \& Kellam, 2003) and young adolescents' self-regulation (Kim \& Brody, 2003), a developmental approach may offer incremental predictive validity over and above approaches based on static variables by adding the predictive value of developmental changes.

\section{Development of oppositionality}

Oppositionality is thought to be relatively common in early adolescence. The literature often depicts young adolescents as rebellious teens that frustrate their parents and teachers by resisting their authority (Abrahamson, Baker, \& Caspi, 2002; Arnett, 1999). Adolescents' problems with authority are thought to be part and parcel of the many biological, cognitive, and social-cognitive changes associated with the onset of adolescence (Paikoff \& BrooksGunn, 1991). Apparent growth in social understanding in early adolescence supports greater autonomy and makes the limitations of adult authority visible (Youniss, 1980). In addition, young adolescents may form new beliefs and goals that do not coincide with those of significant others (Granic et al., 2003). A mismatch between adolescents' expectations and those of significant others and an increased willingness to disagree with those in authority may result in hierarchical power struggles in early adolescence (Bugental, 2000).

The few longitudinal studies available have examined developmental changes in oppositionality across broad age ranges. Nagin and Tremblay (1999) examined developmental changes in oppositionality from childhood to adolescence. A large number of boys were assessed once in kindergarten, and annually between the ages of 10 and 15 years. More recently, Bongers, Koot, van der Ende, and Verhulst (2004) examined developmental changes in oppositionality from early childhood (age 4) to late adolescence (age 18). Both boys and girls were assessed five times with two-year intervals. Contrary to the general expectation, both studies showed a normative decrease in opposition. However, they also revealed substantial interindividual differences in intraindividual change.

The studies mentioned examined developmental changes in oppositionality over long periods of time and used relatively large measurement intervals. Large intervals may obscure short-term changes. Assuming that rather small and rapid changes may characterize the development of oppositionality during early adolescence, we decided to assess oppositionality 
more frequently and with relatively short time intervals. A recent study by Galambos, Barker, and Almeida (2003) focusing on trajectories of change in externalizing and internalizing problems during early adolescence supported this decision. Using longitudinal data collected at five waves during early adolescence, the authors showed that developmental trajectories of externalizing behaviors generally increased and were meaningfully related to dimensions of parenting. These findings suggest that short measurement intervals during early adolescence can provide reliable and precise estimates of intraindividual change. In the present study, we focused on the development of commonly observed oppositional behaviors and emotions during the final years of primary education in a community sample of boys and girls. Selfreports of children's oppositionality were obtained twice a year over a 3-year period spanning the early adolescent years.

\section{Developmental significance of oppositionality}

Two competing hypotheses can be formulated with respect to the developmental significance of oppositionality. The first hypothesis is the risk-hypothesis. This hypothesis states that oppositionality has mainly negative consequences and is therefore related to deviant outcomes. It is based on the assumption that oppositionality reflects a propensity to violate social rules and predisposes children for serious conduct problems. The combined effects of two risk mechanisms may explain why oppositionality is likely to lead to negative outcomes. First, it may be that the adolescent's problems with authority and social rules increase the probability of deviant outcomes in the long run, because the child does not learn to use more adaptive forms of dealing with adult authority. More competent forms are negotiating disagreements or trying to persuade parents or teachers to modify their demands (Bugental, 2000). Because these skills are important in defending one's own interest in future hierarchical interactions at school and at work, a behavioral repertoire that emphasizes opposition can have maladaptive consequences in academic and professional life. Second, oppositionality may start a cycle of maladaptation (c.f. Kokko \& Pulkkinen, 2000). Oppositional behaviors may evoke negative reactions from parents, teachers, or peers, and possibly even disturb the relationship with these significant others. This may in turn increase the child's difficulties, probably resulting in a cycle of opposition and negative responses from the environment (e.g. adult coercion). The cumulative disadvantages and negative consequences of oppositionality could ultimately lead to a pattern of deviant outcomes.

According to the risk-hypothesis, oppositionality leads to a host of difficulties, including poor academic achievement, diminished social support, greater involvement in antisocial behaviors, and child psychopathology. The distinct contribution of opposition to the prediction of juvenile delinquency (Broidy et al., 2003; Langbehn, Cadoret, Yates, Troughton, \& Stewart, 1998) clearly indicates that oppositionality can be regarded as a propensity to violate social rules that could lead to a progression over time into serious antisocial behaviors. Similarly, oppositionality has been shown to predict problems in social functioning (Hastings 
et al., 2000; Laursen, Pulkkinen, \& Adams, 2002) that may well lead to a loss of social support. However, the expected links between, on the one hand, oppositionality and, on the other hand, academic achievement and psychopathology may be less obvious. Nevertheless, it is likely that disruptions of relationships with teachers and classmates affect children's school motivation and thereby their achievement (Ledingham, 1999). The link between oppositionality and child psychopathology can be understood by assuming that deficits in emotion regulation and problem solving skills underlie both the child's oppositionality and psychopathological problems (c.f. Greene \& Doyle, 1999). In short, the risk-hypothesis assumes that oppositionality has detrimental effects only.

The second hypothesis is the competence-promoting hypothesis. According to this hypothesis, the development of oppositionality during early adolescence would be related to positive developmental outcomes. It is based on the assumption that oppositionality during early adolescence reflects the adolescent's striving for greater autonomy and changing conceptions of authority. Because opposition is considered to be a normal developmental phenomenon and the majority of oppositional children do not appear to progress to more serious antisocial behaviors (Hinshaw \& Anderson, 1997), oppositionality may be an indication of the child's developing competencies in the domain of autonomy and the child's changing conceptions of authority rather than a precursor of later antisocial conduct.

Obtaining a degree of independence and gaining the ability to operate in an autonomous manner without being controlled by others are important developmental tasks in early adolescence that are crucial to identity formation (Frick et al., 2003). Success in accomplishing these important developmental tasks during this critical transition period may require some opposition. Oppositionality may engender feelings of control and independence, especially when it enables the child to resolve conflicts to his or her own advantage and to obtain desired freedoms. In line with the supposed beneficial effects of parent-child conflict on adolescents' development (Steinberg, 2000), oppositionality may be competencepromoting behavior. From this theoretical standpoint, opposition is conceived of as constructive behavior in mastering the developmental challenges of early adolescence. According to the competence-promoting hypothesis, oppositional children will turn out to be more autonomous, self-confident, and self-assertive adolescents.

\section{Gender differences}

In recent years, the development of girls' externalizing behaviors has received increasing attention (e.g. Côté, Zoccolillo, Tremblay, Nagin, and Vitaro (2001). This has resulted in a better understanding of gender differences in the prevalence, development, and manifestation of the broad category of externalizing behaviors (Crick \& Zahn-Waxler, 2003), but to date little is known about the role of gender in oppositionality, one of the core constructs of externalizing behavior. The existing studies on the development of oppositionality suggest that boys and girls differ in their mean level of oppositionality, but 
that developmental changes are the same for boys and girls (Bongers et al., 2004; Lahey et al., 2000). Nevertheless, we expect that the development of oppositionality during early adolescence will differ for boys and girls. This expectation is based on the fact that puberty begins one or two years earlier for girls. Because puberty can be expected to trigger many of the changes associated with early adolescence, the expectedly associated increase in oppositionality during this period may be earlier visible in girls than in boys.

The developmental significance of oppositionality during early adolescence is also expected to vary for boys and girls. There is little reason to expect gender differences in the hypothesized competence-promoting effect of oppositionality, but the risks associated with oppositionality may be greater for girls. Early adolescence may be a period in which girls are particularly vulnerable (Rudolph, 2002; Wångby et al., 1999). It has been suggested that girls generally behave in a "nice" manner and hide most of their negative feelings during childhood, but that this situation changes markedly as they enter adolescence. There is empirical evidence (Crick \& Zahn-Waxler, 2003) that adolescence is a period in which girls increasingly express their feelings, assert their own opinions, and behave in rebellious ways. The sharp contrast between the "nice girl" in childhood and a rebellious teenage girl may make that significant others in the child's life respond more fiercely to oppositional behavior in girls than in boys. Intense negative reactions, such as hostility or excessive behavioral restrictions, can further fuel girls' oppositionality and lead to an aggravation over time that could well result in serious adjustment problems for girls. The detrimental impact of these negative reactions may even be strengthened by girls' heightened level of interpersonal sensitivity (Leadbeater et al., 1999; Rudolph, 2002).

Finally, the specific types of adjustment problems may vary for boys and girls. It may be that oppositional behavior among girls is more likely to be associated with psychopathology and relationship problems, whereas oppositional behavior among boys is more likely to be related to poor academic achievement. Gender differences in the specific types of adjustment problems are supported by recent findings by Moffitt et al. (2001). Their results indicate that early antisocial behavior has disruptive effects in young adulthood for both males and females. However, young men appear to be particularly affected in the domain of education and employment, whereas females appear to be mainly at risk for mental and physical health problems and difficulties in interpersonal relationships.

\section{The present study}

The purpose of the present study was to evaluate two competing hypotheses regarding the developmental significance of oppositionality, i.e. the risk-hypothesis and the competence-promoting hypothesis. In order to contrast these hypotheses, we examined the relations between developmental trajectories of oppositionality and a wide variety of both positive and negative developmental outcomes. Boys and girls were assessed in a longitudinal study with six measurement occasions during early adolescence and half-year intervals 
between occasions. We used the Amsterdam Scale of Oppositionality (ASO; Hoffenaar \& Hoeksma, 2002), a self-report instrument that measures the full range of oppositional responses to repeatedly assess oppositionality. In addition, we took advantage of recent methodological advances for establishing developmental change (Collins \& Sayer, 2001) and longitudinal prediction (Hoeksma \& Knol, 2001) to examine the relation between individual differences in developmental change and developmental outcomes. The present study examined developmental trajectories of oppositionality and their link to a variety of outcomes for each gender separately. Separate analyses for boys and girls are advisable given that past results have indicated gender differences in the level of oppositionality (Hoffenaar \& Hoeksma, 2002) and the expected gender differences in the development of oppositionality because of the impact of pubertal timing.

The variety of developmental outcomes included academic achievement, autonomy, self-esteem, social support, social skills, antisocial behaviors, and child psychopathology. These adjustment measures were assessed by using multiple informants and at a point in time that coincides with the transition from elementary school to secondary school. Around the age of 12 , young adolescents are assumed to be ready for new academic challenges. It is also assumed that they have reached a certain level of social and emotional maturity that makes them ready for a new school system. Therefore this 'natural' transition provides a good opportunity to examine the relation between prior developmental changes in oppositionality and outcomes reflecting adolescents' success or failure in developmental tasks salient to early adolescence.

The outcomes represent aspects of the broad constructs of academic competence, social competence, and psychological adjustment (e.g. Brody, Murry, Kim, \& Brown, 2002). Child psychopathology was also included in order to examine the relation between oppositionality and psychiatric risk. All of these broad domains of the child's functioning are clearly interrelated, but Masten et al. (1995) found empirical support for treating academic competence, peer social acceptance, and conduct as distinct dimensions of positive adjustment. Antisocial behaviors and child psychopathology can be regarded as distinct dimensions of negative adjustment.

\section{Method}

\section{Participants}

Participants were first assessed in Grade 6 as part of a longitudinal investigation of children's development in early adolescence. Schools were drawn from a register of all Dutch primary schools. Probability of inclusion for each school was inversely proportional to the mean school size per province. Schools were contacted by phone. Approximately one in three schools refused participation for various reasons, none of these being related to the variables of interest. The main reasons given were: "We are too busy" and "We recently participated in another research project". When a school refused participation, the next school in the sample 
register replaced this school. Teachers and school principals of 17 schools agreed to participate.

The total sample was created in two waves. After the first year of data collection participating schools were asked whether the next group of 6th graders could also participate in the research. Ten of the original 17 schools agreed. Because MANOVA revealed no significant differences on important sample characteristics, the two cohorts were combined. Signed informed parental consent statements were obtained for the majority of children within classrooms (about 90\%). This resulted in a representative sample of 498 Dutch 6th Grade pupils from 27 different classes in 17 different schools. Table 1 displays the sample characteristics. The sample was fairly evenly divided according to gender and demographically diverse.

Table 1.

Sample characteristics $(N=498)$

\begin{tabular}{|c|c|c|}
\hline \multirow{2}{*}{\multicolumn{3}{|c|}{ Characteristic }} \\
\hline & & Gender \\
\hline Boys & \multicolumn{2}{|c|}{$48.6 \%$} \\
\hline Girls & \multicolumn{2}{|c|}{$51.4 \%$} \\
\hline \multicolumn{3}{|l|}{ Mean age at occasion 1} \\
\hline Boys (yrs.) & \multicolumn{2}{|c|}{$9.9(.46)$} \\
\hline Girls (yrs.) & \multicolumn{2}{|c|}{$9.7(.42)$} \\
\hline \multicolumn{3}{|l|}{ Family size } \\
\hline Mean number of siblings & \multicolumn{2}{|c|}{$1.6(1.02)$} \\
\hline \multicolumn{3}{|l|}{ Family composition } \\
\hline Two-parent families & \multicolumn{2}{|c|}{$91.6 \%$} \\
\hline Single-parent families & \multicolumn{2}{|c|}{$7.5 \%$} \\
\hline Other & \multicolumn{2}{|c|}{$0.9 \%$} \\
\hline Highest educational level & Mother & Father \\
\hline Vocational training & $\overline{25.5 \%}$ & $\overline{22.7 \%}$ \\
\hline Secondary school & $55.0 \%$ & $46.1 \%$ \\
\hline Higher education & $19.5 \%$ & $31.2 \%$ \\
\hline Mean weekly working hours & $12.9(12.2)$ & $40.9(9.0)$ \\
\hline Mean age (yrs.) & $38.6(5.0)$ & $41.2(4.9)$ \\
\hline
\end{tabular}

Note. Standard deviations in parentheses.

Data collection started in the fall of 1999 and ended in the spring of 2003. Self-reports of oppositionality were collected twice a year over a period of three years (the 6th, 7th, and 8th grades). Developmental outcomes were assessed at the end of Grade 8. 


\section{Measures}

Oppositionality. Oppositionality was measured by means of self-reports on the Amsterdam Scale for Oppositionality (ASO; Hoffenaar \& Hoeksma, 2002). The 30 items of the ASO represent emotional and behavioral responses in situations with parents, teachers, and peers. Each item consists of a description of the situation (interactions with parents, teachers or peers), followed by two response alternatives: one oppositional alternative and one non-oppositional response alternative. These alternatives were either emotional responses or behavioral responses. The six possible combinations of responses and situations are each represented by five forced-choice items, resulting in a total of 30 items.

An example of an item reflecting a behavioral response in interactions with parents is (with assigned scores between brackets): When my dad or mum tells me to help, I do it (0)/I pretend I haven't heard (1). An item reflecting oppositional feelings towards the teacher is: When the teacher punishes me, I feel sad (0)/I get angry (1). An item reflecting an emotional response in situations with peers: When another child is looking for trouble, I feel like fighting (1)/I want to walk away (0).

Summing the responses to all items gives a total score for oppositionality (possible range from 0 to 30). In a previous study (Hoffenaar \& Hoeksma, 2002), the structural validity of the ASO was investigated in a cross-sectional sample of 1,158 children. Cronbach's alpha for the total oppositionality score was .86 , indicating adequate reliability. Test-retest stability for a sample of children who were tested twice with a two-week interval was .89 $(N=150)$. In the present sample, the alpha for oppositionality varied across measurement occasions from .88 to .90 . The criterion-related validity of the ASO (Hoffenaar, Hoeksma, and Koot, submitted) was supported by substantial positive correlations of the total oppositionality score with parent and teacher ratings of conduct problems $(\mathrm{r}=.41, N=505)$ and hyperactivity $(\mathrm{r}=$ $.38)$, and a negative correlation with adult ratings of prosocial behavior $(\mathrm{r}=-.36)$.

Academic achievement. Language proficiency and math proficiency were measured using tests developed by the National Institute for Educational Measurement (CITO). These tests are routinely administered in the last year of primary education. The reliabilities have been found to range between .85 and .86 for the language tests and between .95 and .97 for the math tests, respectively (Driessen, 2002). Teachers provided an overall score for academic achievement, which is the sum of these two tests.

Autonomy. Autonomy was measured with the Adolescent Autonomy Questionnaire. This self-report instrument is based on a conceptual analysis of autonomy and its validity was empirically demonstrated (Noom, Dekovic, \& Meeus, 2001). The most recent version of this self-report instrument (obtained from the author) consists of 18 items that the adolescent is asked to rate on a five-point scale, ranging from "describes me poorly" to "describes me very well". The items of the questionnaire refer to three aspects of autonomy, i.e. attitudinal autonomy (the ability to define goals), emotional autonomy (a general feeling of confidence and trust in making choices), and functional autonomy (the ability to develop a strategy for 
achieving goals). Because all items are also thought to refer to a general concept of autonomy, we choose to use the more reliable total score obtained by summing over all 18 items. Internal consistency for the composite autonomy score was .78.

Self-esteem. Adolescents reported on their own self-esteem using the global self-worth subscale of the Self-Perception Profile for Children (SPPC) developed by Harter (1988). This subscale includes six items that ask adolescents whether they are happy with their life and how they are, and if they like themselves. In contrast to the original two-step, forced-choice format, we used a modified response format that is assumed to be easier for children and adolescents (see also Brody, Murry, Kim, \& Brown, 2002). Each item consisted of one statement describing a certain type of children. Adolescents were asked to what extent the description fitted their self-perception by choosing one of the following alternatives: "I am not like these children", "I am a bit like these children", "I am most like these children", and "I am precisely like these children". Each item was scored from 1 to 4 and the six item scores were summed into a global self-worth score. In the present sample, Cronbach's alpha for global self-worth was .85.

Perceived Social Support. The Social Support Scale for Children (Harter, 1985) was used to assess adolescent perceived support from parents, teachers, classmates, and close friends. The items have the same format as the previously described Self-Perception Profile for Children. An example item reflecting support from parents is: "Some kids have parents who treat their child like a person who really matters". Adolescents read the statement and subsequently rate how much they are like the type of adolescent described. Subscales reflecting each source of social support consist of six items each. Items are scored from 1 ("I am not like these children") to 4 ("I am exactly like these children"). Subscale scores are obtained by summing relevant items. In the present sample, the Cronbach's alphas were .65 , $.66, .71$, and .66 for perceived support from parents, teachers, classmates, and close friends, respectively. These low reliabilities likely mean that relations with other variables will be attenuated. Although this is undesirable, these scales were retained because the link between developmental trajectories of oppositionality and perceived support from each separate source was thought to be of substantive interest.

Social Skills. Using the parent and teacher forms of the Social Skills Rating System (SSRS; Gresham \& Elliot, 1990), we assessed adolescents' social skills. The SSRS assesses assertion (initiating behaviors, such as asking others for information), cooperation (helping others, sharing materials, and complying with rules and directions), self-control (adequate behavior in conflict situations, such as responding appropriately to teasing), and responsibility (the ability to communicate with adults and regard for property or work). The two forms have different items to reflect the unique and specific nature of social skills in different settings (home and school). In addition, only parents rate the social skill of responsibility and the teacher and parent forms of the SSRS differ in length (30 and 38 items, respectively). Teachers and parents rate the frequency of each social skill item on a three-point Likert-type 
scale (never, sometimes, very often). In a review of six different scales for social skills assessment, Demaray et al. (1995) recommended the SSRS because of its strong reliability and validity. In the present sample, the Cronbach's alphas were $.88, .89$, and .90 for teacher ratings of assertion, cooperation, and self-control, respectively. The alphas for parent ratings of assertion, cooperation, self-control, and responsibility were .59, .76, .78, and .60, respectively.

Antisocial behaviors. Self-reports of illegal and norm violating behaviors were obtained by using the Self-Report Early Delinquency Scale (SRED; Moffitt \& Silva, 1988). The SRED is specifically designed to capture antisocial behaviors in young adolescents. Its reliability and validity were considered adequate (Moffitt \& Silva, 1988). In addition, the instrument was one of the measures used in the Dunedin Multidisciplinary Health and Development Study used to study sex differences in antisocial behavior (Moffitt et al., 2001). From the original SRED, 31 items were selected. The selection covered a wide range of antisocial behaviors, including property damage (vandalism, car damage, making graffiti), interpersonal conflict (fighting, carrying weapons, running away from home, truancy, expulsion from school), theft (stolen something, bought or sold stolen goods), and substance use (used marijuana, cigarette smoking, alcohol consumption). In the present study, the frequency scores for each item of the SRED were dichotomized as "once or more than once" versus "never". This decision was based on findings of Osgood, McMorris, and Potenza (2002) indicating that differentiating among frequencies of antisocial behaviors adds little extra information to the distinction between offenders and non-offenders. Cronbach's alpha for the resulting scale was .87 in the present sample.

Child psychopathology. The Strength and Difficulties Questionnaire (SDQ, Goodman, 1997) was used to assess symptoms of child psychopathology. Identical versions of the questionnaire were filled out by parents and teachers. Children themselves filled out the selfreport version of the SDQ. A total difficulties score (possible range from 0 to 40) is derived by summing the scores of four subscales of the SDQ, i.e. conduct problems, hyperactivity, emotional problems, and peer problems. Each of these subscales contains five items. Parents, teachers, and children themselves rated identical or nearly identical items on a three-point scale $(0=$ not true, $1=$ somewhat true, $2=$ certainly true). We choose to use the total difficulties score of the SDQ because this more global index of the child's mental health problems is more reliable than the subscale scores.

The psychometric properties of the SDQ were recently investigated in a representative sample of 10,438 children aged between 5 and 15 years (Goodman, 2001). The results indicated satisfactory reliability and validity of the SDQ scores. In addition, the total difficulties score was strongly associated with the presence of psychiatric disorder. The odds ratios for having a psychiatric disorder were 6.6 for the self-reported difficulties, 15.2 for parent-rated difficulties, and 13.5 for teacher-rated difficulties. Studies of Dutch children and adolescents provided further evidence on the internal consistency, test-retest stability, inter- 
informant agreement, and concurrent validity of the SDQ scales (Muris et al., 2003; Widenfelt, Goedhart, Treffers, \& Goodman, 2003). In the present sample, the Cronbach's alphas for the total difficulties scale were .81 for the self-report version of the SDQ, .81 for the parent ratings, and .86 for the teacher ratings.

\section{Procedure}

A research assistant or the first author visited the schools twice a year (in the fall and in the spring). On each occasion, a set of questionnaires (the ASO, a short questionnaire on children's emotional experiences, and the SDQ) was group administered in the classroom. At the last measurement occasion, the set of questionnaires included several additional measures to obtain self-reported outcomes. After a short introduction, questionnaires were distributed and completed individually by all adolescents. The majority completed all questionnaires in less than thirty minutes. At the last measurement occasion, completion generally took about forty-five minutes. After completion, children received a freepost pre-addressed envelope containing parental questionnaires with the request to give this envelope to their parents. Children and their parents were assured confidentiality and only their ID number appeared on the questionnaires or envelopes. Teachers also received a pre-addressed envelope containing questionnaires for each individual child.

\section{Statistical Analyses}

To assess the relationship between developmental changes in oppositionality and developmental outcomes, we used multilevel modeling. Goldstein (1989) first introduced the multilevel model for longitudinal prediction and showed how the model could be applied to the prediction of adult physical height from early growth. More recently, Hoeksma and Knol (2001) showed how the model can be used to test predictive developmental hypotheses involving psychological data. They conclude that the multilevel prediction model offers a more valuable tool to developmental psychologists than more conventional approaches to longitudinal prediction.

The multilevel model offers several advantages over approaches generally used for the purpose of prediction, such as regression analysis. Most importantly, the multilevel model for longitudinal prediction explicitly takes the developmental process into account and links it directly to the outcome. The proposed method further offers the advantage that all available data are used to describe the development of oppositionality and its relation to the outcome variable. Participants with missing data on the repeated measurements are allowed in estimating individual developmental trajectories for oppositionality. Missing data on the developmental outcomes are also handled within the model. The latter means that even if data on the outcome are not available, the prediction model is still strengthened by including all available data on the developmental process. 
The model contains two parts. The first part describes the repeated measurements pertaining to the development of oppositionality (c.f. Bryk \& Raudenbush, 1987; Hoeksma \& Koomen, 1992; Boyle \& Willms, 2001). The second part contains the developmental outcome described as deviations from its mean. In the first part of the model, the individual developmental trajectory of oppositionality of each adolescent was modeled by two parameters, i.e. an intercept and a slope parameter. Because the parameters for the models were estimated with the centering age set at 11 (middle of early adolescence), the intercept refers to the mean level of oppositionality during early adolescence. The slope refers to the rate of change during this period. The model links both the mean level and the rate of change to the developmental outcome. To illustrate, a positive correlation between the rate of change and the outcome would indicate that increases in oppositionality with age are related to a higher score on the outcome.

To account for the fact that children within school classes are subject to the same contextual influences, separate levels for the school class and the individual child were specified (Boyle \& Willms, 2001; Snijders \& Bosker, 1999). Similarly, the dependency between multiple ratings by the same teacher was modeled by allowing teacher outcomes to vary across school classes and children. Differences between school classes were modeled at level 3, differences between children at level 2, and residual variation was estimated at level 1. Residual variation consists of both error related to the specific time of measurement and measurement error.

The multilevel model for longitudinal prediction was estimated using the IGLS algorithm implemented in Mlwin 1.10 (Rasbash et al., 2000). After the model linking the developmental trajectories to the developmental outcomes is fitted, it is used to predict the individual scores on the outcomes. For that purpose, so-called empirical Bayes estimates are used (Goldstein, 2003). These estimated predicted values are based on the repeated measurements pertaining to the development of oppositionality. The prediction accuracy is indicated by the Pearson correlation between the predicted and observed scores (details can be found in Hoeksma and Knol, 2002).

\section{Missing data}

At each measurement occasion, participants were lost because of missed items on the ASO and attrition. The average number of measurement occasions available was 5.30 $(S D=1.17)$. For $62.4 \%$ of the children, complete data on all six occasions were available. For most children with incomplete data, only one occasion was missing (22.1\%). Smaller percentages of children had missing data on two $(6 \%)$, three $(3.8 \%)$, or more $(5.6 \%)$ measurement occasions. Because missing data at the level of the individual are allowed in multilevel modeling (Snijders \& Bosker, 1999), all children were retained in the longitudinal analyses. 
Data on self-reported outcomes were available for varying numbers of adolescents, ranging from $73.8 \%$ for autonomy to $84.7 \%$ for self-esteem. Missing data were due to missed items on the questionnaire concerned (varying between $3.6 \%$ and $14.5 \%$ ) or failure to participate at the last measurement occasion (11.7\%). Due to parental nonresponse and study losses data on parent-reported outcomes were available for $58.4 \%$ of the children. Data on teacher-reported outcomes were available for about $65 \%$ of the children. Here, non-response by 8 of the 27 teachers accounted for $23.3 \%$ of the missing data and study losses accounted for another $11.7 \%$ of the missing data.

Although high nonresponse rates are not uncommon, they do raise the question whether the studied sample adequately represents the study population. To examine the effect of nonresponse, we focused on possible bias in the individual mean levels of oppositionality and rates of change. We first compared the participants with complete data and the participants with developmental data but no outcome data. The effect of nonresponse varied across informants and specific outcomes. Although several significant differences were found in the mean level of oppositionality and rate of change, the magnitude of these differences was small and never accounted for more than $2 \%$ of the variance. In addition, we calculated the actual amount of bias by applying the formula presented by Gerrits, van den Oord, and Voogt (2001). According to these authors, bias depends on the nonresponse rate and the effect size of the observed difference. Estimated bias ranged from .003 to .008 , indicating that the difference between the "observed" mean in the final sample of participants and the true "unobserved" mean in the total sample was .008 standard deviation at most. This suggests that the actual bias introduced by attrition and missing values was negligible.

\section{Results}

\section{Descriptive statistics}

Table 2 displays observed means of oppositionality across the six measurement occasions. The mean level of oppositionality increased slightly with age. Both the mean level and standard deviation (reflecting interindividual differences) were almost twice as large for boys compared to girls. The greater interindividual differences in boys' oppositionality supported our decision to estimate the models separately for boys and girls.

Table 3 displays the observed means of the outcome measures. Comparison of the means for boys and girls revealed only one striking gender difference. Boys appeared to report substantially more antisocial behaviors than girls $(d=.15)$. Boys and girls had very similar levels of autonomy and had similar academic achievement scores. Girls appeared to experience more social support and were generally rated somewhat higher on social skills. In addition, boys scored higher on psychopathology ratings. Although these gender differences were apparent, effect sizes for these differences were small (ranging from $d=.02$ for selfreported psychopathology to $d=.07$ for teacher-rated cooperation). 
Table 2.

Descriptive statistics for the developmental data; repeated measurements of oppositionality.

\begin{tabular}{cccccc}
\hline & \multicolumn{2}{c}{$\underline{N}$} & \multicolumn{2}{c}{$M(S D)$} & \\
Occasion & Boys & Girls & Boys & Girls \\
\hline 1 & 230 & 249 & $7.31(6.11)$ & $3.60(3.53)$ \\
2 & 221 & 240 & $7.77(6.06)$ & $3.92(4.07)$ \\
3 & 208 & 217 & $7.55(5.78)$ & $3.71(3.84)$ \\
4 & 211 & 216 & $8.46(6.50)$ & $4.57(4.29)$ \\
5 & 207 & 209 & $8.01(6.50)$ & $4.05(4.52)$ \\
6 & 211 & 221 & $7.92(5.94)$ & $4.95(4.92)$ \\
\hline
\end{tabular}

In order to evaluate the dependency between the outcome variables, Table 3 displays their intercorrelations. The magnitudes of the correlations across different domains were generally low to moderate. The intercorrelations among different outcome domains rated by the same source were fairly strong, ranging from $|.07|$ to $|.55|$ for self-reports, from $|.34|$ to $|.59|$ for parent reports, and from $|.45|$ to $|.78|$ for teacher reports. Cross-informant correlations ranged from .20 to .27 for parent and teacher ratings of children's social skills and from .41 to .50 for the child, parent, and teacher ratings of psychopathology. In sum, the various outcome measures appear to represent distinct, but overlapping dimensions.

\section{Development of oppositionality}

Individual developmental trajectories were modeled by two parameters, i.e. an intercept and a slope parameter. Adding a quadratic term or 'acceleration' parameter to the models did not significantly improve the model fit (boys: $\chi^{2}(2)=1.56$, n.s.; girls: : $\chi^{2}(2)=1.19$, n.s). As a consequence, we modeled the development of oppositionality as a linear function of age. As noted before, the intercept refers to the mean level of oppositionality during early adolescence and the slope refers to the rate of change during this period.

Models were fitted for boys and girls separately. Table 4 displays the parameter estimates of the models. The fixed parameters describe the average developmental trajectory. The estimated mean level of oppositionality (intercepts) during early adolescence was 7.88 $(S E=.47)$ for boys and $4.28(S E=.28)$ for girls, suggesting that boys rated themselves on average nearly twice as oppositional as girls. The positive rates of change (slopes) indicate that the mean developmental trajectory increased with age for both boys and girls. On average, boys' scores increased $.32(S E=.16)$ points per year on the oppositionality scale and girls' scores increased $.60(S E=.13)$ points. Thus, the mean developmental trajectory of oppositionality for girls appears to be twice as low, but at the same time twice as steep as the mean trajectory for boys. 
Table 3. Descriptive statistics for developmental outcomes and intercorrelations

\begin{tabular}{|c|c|c|c|c|c|c|c|c|c|c|c|c|c|c|c|c|c|c|}
\hline Developmental outcomes & 1 & 2 & 3 & 4 & 5 & 6 & 7 & 8 & 9 & 10 & 11 & 12 & 13 & 14 & 15 & 16 & 17 & 18 \\
\hline Boys: Mean & 536.8 & 65.7 & 20.1 & 21.1 & 18.4 & 18.6 & 19.0 & 7.2 & 9.8 & 15.7 & 12.1 & 12.4 & 14.7 & 7.6 & 13.7 & 14.4 & 14.1 & 8.9 \\
\hline Girls: Mean & 536.0 & 65.2 & 19.6 & 21.9 & 19.2 & 19.7 & 20.5 & 4.1 & 8.4 & 16.0 & 13.3 & 13.1 & 15.0 & 6.6 & 14.9 & 16.6 & 15.9 & 6.5 \\
\hline Boys: $S D$ & 8.9 & 8.7 & 3.5 & 2.8 & 3.4 & 3.4 & 3.4 & 4.1 & 5.9 & 2.5 & 3.2 & 3.2 & 2.4 & 5.1 & 4.1 & 4.1 & 4.2 & 6.4 \\
\hline Girls: $S D$ & 9.5 & 9.5 & 3.9 & 2.4 & 3.2 & 3.0 & 3.1 & 3.1 & 5.5 & 2.3 & 3.5 & 3.2 & 2.4 & 5.2 & 4.3 & 3.7 & 3.9 & 5.9 \\
\hline 1. Academic Achievement & - & & & & & & & & & & & & & & & & & \\
\hline 2. Autonomy & .23 & - & & & & & & & & & & & & & & & & \\
\hline 3. Self-esteem & .24 & .51 & - & & & & & & & & & & & & & & & \\
\hline Social support & & & & & & & & & & & & & & & & & & \\
\hline 4. Parents & .24 & .35 & .38 & - & & & & & & & & & & & & & & \\
\hline 5. Teachers & .21 & .12 & .23 & .39 & - & & & & & & & & & & & & & \\
\hline 6. Classmates & .22 & .38 & .52 & .37 & .21 & - & & & & & & & & & & & & \\
\hline 7. Close friends & .25 & .31 & .37 & .37 & .22 & .61 & - & & & & & & & & & & & \\
\hline 8. Antisocial behaviors & -.13 & -.07 & -.24 & -.29 & -.31 & -.19 & -.19 & - & & & & & & & & & & \\
\hline 9. Psychopathology & -.33 & -.52 & -.55 & -.35 & -.19 & -.55 & -.42 & .30 & - & & & & & & & & & \\
\hline \multicolumn{19}{|l|}{ Social skills } \\
\hline 10. Assertion & .16 & .24 & .13 & .19 & .03 & .31 & .25 & .02 & -.19 & - & & & & & & & & \\
\hline 11. Cooperation & .23 & .22 & .15 & .23 & .14 & .24 & .19 & -.11 & -.26 & .38 & - & & & & & & & \\
\hline 12. Self-control & .35 & .28 & .20 & .21 & .06 & .23 & .18 & -.17 & -.36 & .36 & .60 & - & & & & & & \\
\hline 13. Responsibility & .29 & .20 & .13 & .23 & .14 & .15 & .11 & -.08 & -.17 & .47 & .55 & .56 & - & & & & & \\
\hline 14. Psychopathology & -.41 & -.36 & -.34 & -.19 & -.12 & -.42 & -.33 & .19 & .50 & -.36 & -.45 & -.59 & -.34 & - & & & & \\
\hline \multicolumn{19}{|l|}{ Social skills } \\
\hline 15. Assertion & .30 & .21 & .15 & .22 & .23 & .25 & .31 & -.07 & -.26 & .27 & .29 & .30 & .28 & -.33 & - & & & \\
\hline 16. Cooperation & .46 & .12 & .16 & .19 & .31 & .16 & .21 & -.30 & -.31 & .06 & .20 & .23 & .13 & -.29 & .52 & - & & \\
\hline 17. Self-control & .37 & .14 & .15 & .18 & .25 & .22 & .26 & -.18 & -.28 & .20 & .21 & .26 & .19 & -.37 & .77 & .66 & - & \\
\hline 18. Psychopathology & -.45 & -.19 & -.23 & -.25 & -.32 & -.31 & -.32 & .21 & .41 & -.23 & -.21 & -.28 & -.16 & .42 & -.65 & -.78 & -.76 & - \\
\hline
\end{tabular}

Note. Correlations greater than \pm .07 are significant at $p<.05$; correlations greater than \pm .14 are significant at $p<.01$ 

The random part of the models shows the extent to which the developmental trajectories varied between school classes, individuals and measurement occasions. The average proportion of the total variance in oppositionality that could be attributed to differences between school classes was $7.7 \%$ for boys and $4.7 \%$ for girls. The between-person variance in the individual mean level of oppositionality was $21.17(S E=2.28)$ for boys and $9.80(S E=1.04)$ for girls. The variance in the rate of change was $3.10(S E=.54)$ for boys and $2.71(S E=.38)$ for girls. The results reveal quite substantial heterogeneity in the developmental trajectories of both boys and girls. The individual variability in the mean level of girls' oppositionality appeared to be approximately half of the variability in the mean level of boys' oppositionality.

Table 4.

Fixed parameter estimates (Top) and variance- and covariance parameters (Bottom) for the multilevel models describing the development of oppositionality.

\begin{tabular}{|c|c|c|}
\hline & Boys & Girls \\
\hline & \multicolumn{2}{|c|}{ Fixed part } \\
\hline Intercept & $7.88(.47)$ & $4.28(.28)$ \\
\hline Slope & $.32(.16)$ & $.60(.13)$ \\
\hline
\end{tabular}

School classes (level 3)

Random part

$\begin{array}{lcc}\text { Intercept } & 3.08(1.60) & .92(.54) \\ \text { Individuals (level 2) } & & \\ \text { Intercept } & 21.17(2.28) & 9.80(1.04) \\ \text { Slope } & 3.10(.54) & 2.71(.38) \\ \text { Intercept/Slope } & 1.11(.78) & 2.45(.47) \\ \text { Measurement occasions (level 1) } & 10.19(.50) & 5.55(.27) \\ \quad \text { Residual variance } & \end{array}$

Note. Standard errors for parameter estimates are displayed in parentheses; the intercept was coded at the middle of the period studied (age 11) and therefore refers to the mean level.

\section{Prediction of developmental outcomes}

Each estimated multilevel prediction model combined the model describing the development of oppositionality with one of the developmental outcomes. Table 5 displays the correlations between the parameters referring to the developmental trajectories (intercept and slope) and the outcome for boys and girls. The first column of correlations refers to the relations between the individual mean levels of oppositionality during early adolescence and the outcomes. The second column of correlations refers to the relations between the individual rates of change in oppositionality and the outcomes. Positive correlations between the rates of change and an outcome indicate that increasing trajectories are related to higher scores on the outcome than less increasing or decreasing trajectories. Finally, the last column refers to the 
prediction accuracy expressed by the correlation between predicted individual outcome scores and observed scores. They were calculated after the models were estimated.

Table 5.

Relation between linear change parameters describing the development of oppositionality and later outcomes for boys and girls

\begin{tabular}{|c|c|c|c|c|c|c|}
\hline & \multicolumn{3}{|c|}{ Boys } & \multicolumn{3}{|c|}{ Girls } \\
\hline & Intercept & Slope & Accuracy & Intercept & Slope & Accuracy \\
\hline Academic achievement & $-.34 * *$ & -.05 & $.31 * *$ & $-.27 * *$ & $-.38 * *$ & $.29 * *$ \\
\hline Autonomy & -.05 & .00 & .05 & $-.17 *$ & .01 & $.15^{*}$ \\
\hline Self-esteem & $-.28 * *$ & -.03 & $.26 * *$ & $-.26^{* *}$ & $-.23^{*}$ & $.29 * *$ \\
\hline \multicolumn{7}{|l|}{ Social support } \\
\hline Parent & $-.39 * *$ & -.06 & $.36 * *$ & -.15 & -.12 & $.15^{*}$ \\
\hline Teacher & $-.27 * *$ & -.15 & $.28 * *$ & $-.42 * *$ & $-.32 * *$ & $.41 * *$ \\
\hline Classmates & $-.19 * *$ & .00 & $.17^{*}$ & $-.17 *$ & $-.17 *$ & $.18^{*}$ \\
\hline Close friends & $-.18 *$ & -.09 & $.18^{*}$ & -.15 & -.12 & .13 \\
\hline Antisocial behaviors & $.54 * *$ & $.27 * *$ & $.50 * *$ & $.55 * *$ & $.41 * *$ & $.50 * *$ \\
\hline Psychopathology & $.34 * *$ & .12 & $.35 * *$ & $.40 * *$ & $.32 * *$ & $.36^{* *}$ \\
\hline \multicolumn{7}{|l|}{ Social skills } \\
\hline Assertion & $-.23 * *$ & .08 & $.24 * *$ & $-.25 * *$ & -.01 & $.23 * *$ \\
\hline Cooperation & $-.13 * *$ & .06 & .13 & $-.24 * *$ & -.09 & $.18^{*}$ \\
\hline Self-control & -.17 & .08 & $.21 *$ & $-.42 * *$ & -.14 & $.35 * *$ \\
\hline Responsibility & -.07 & .13 & .15 & $-.35 * *$ & -.17 & $.29 * *$ \\
\hline Psychopathology & $.26^{* *}$ & -.15 & $.27 * *$ & $.38 * *$ & .14 & $.32 * *$ \\
\hline \multicolumn{7}{|l|}{ Social skills } \\
\hline Assertion & $-.22 * *$ & -.19 & $.22 * *$ & -.10 & $-.28 * *$ & $.24 * *$ \\
\hline Cooperation & $-.41 * *$ & $-.30 * *$ & $.43 * *$ & $-.31 *$ & $-.52 * *$ & $.44^{* *}$ \\
\hline Self-control & $-.24 * *$ & -.15 & $.27 * *$ & $-.31 * *$ & $-.31 * *$ & $.33 * *$ \\
\hline Psychopathology & $.33 * *$ & .18 & $.34 * *$ & $.29 * *$ & $.54 * *$ & $.47 * *$ \\
\hline
\end{tabular}

Note. ${ }^{*} p<.05,{ }^{* *} p<.01$; Columns 2 and 5 (intercept) contain the correlation between the mean level of oppositionality and the outcome, columns 3 and 6 (slope) contain the correlation between the rate of change and the outcome, and columns 4 and 7 (accuracy) contain the correlation between the predicted value and observed score on the outcome.

Boys. Table 5 (first column of correlations) shows that the individual mean levels of oppositionality were significantly and negatively related to academic achievement, indicating that boys with high mean levels of oppositionality across the 3-year period did less well academically at the end of elementary school. Developmental changes in oppositionality (see second column of correlations, Table 5) were not significantly related to academic 
achievement. The prediction accuracy of .31 (see third column of correlations) corresponds to a medium effect size (c.f. Cohen, 1988).

The results (Table 5) revealed a consistent pattern for the prediction of self-reported outcomes. Individual mean levels of oppositionality were negatively related to indices of positive adjustment and positively related to indices of negative adjustment. Developmental changes in oppositionality were not significantly related to self-reported outcomes, except for antisocial behaviors. Boys who were more oppositional during early adolescence appeared to think less positively of themselves, perceived less support from several sources, and experienced more mental health problems. In addition, boys with high levels of oppositionality during early adolescence and those whose level of oppositionality increased during this period were likely to report greater involvement in antisocial behaviors at the end of elementary school. Results with regard to the prediction of self-reported autonomy were an exception to the general pattern described above. Interindividual differences in the development of oppositionality were not related to autonomy. The prediction accuracy was .26 for self-esteem and ranged from .17 for support from close friends to .36 for social support from parents, corresponding to low and medium effect sizes. The prediction accuracy for antisocial behaviors was .50 and .35 for psychopathology, corresponding to a large effect size and a medium effect size, respectively.

The results (Table 5) also revealed a very consistent pattern for the prediction of outcomes obtained by parent and teacher reports. Individual mean levels of oppositionality appeared to be negatively related to social skills and positively related to child psychopathology. Boys with high oppositionality scores were likely to be rated as being less assertive, uncooperative and low on self-control (teacher ratings only). In addition, boys who were more oppositional during early adolescence were at increased risk for mental health problems. Developmental changes in oppositionality were only related to one of the teacherrated social skills. The significant negative correlation between the rates of change of oppositionality and teacher-rated cooperation indicates that boys who became increasingly oppositional were likely to be rated by their teacher as uncooperative. Developmental trajectories of oppositionality predicted parent-rated assertion with an accuracy of .24 and self-control with an accuracy of .21, corresponding to low effect sizes. The prediction accuracy for teacher-rated social skills ranged from .22 for assertion to .43 for cooperation, corresponding to low and medium effect sizes. The prediction accuracy for psychopathology was .27 for parents' judgments and .34 for teachers' judgements, indicating medium effect sizes.

Girls. The right part of Table 5 reveals that the individual mean levels and rates of change of oppositionality were significantly and negatively related to academic achievement. Girls with high and increasing levels of oppositionality appeared to score lower on the math and language proficiency tests administered in the last year of elementary school. The 
magnitude of the correlation between predicted and observed scores (prediction accuracy) corresponds to a medium effect size.

The development of oppositionality during early adolescence appeared to be negatively related to most of the self-reported outcomes reflecting positive adjustment. Individual mean levels of oppositionality appeared to be significantly and negatively related to autonomy. Girls who were more oppositional during early adolescence appeared to feel less autonomous. Both the individual mean levels and rates of change were significantly and negatively related to self-esteem and social support from teachers and classmates, indicating that both high levels of oppositionality and increasing levels of oppositionality were followed by more negative perceptions and feelings of self-worth and less social support from teachers and classmates. The individual developmental trajectories of oppositionality were not significantly related to self-perceived social support from parents and close friends. The prediction accuracy was .15 for autonomy, .24 for self-esteem, and .41 for teacher support corresponding to small to medium sized prediction effects.

Interindividual differences in the development of oppositionality appeared to be positively related to the self-reported outcomes reflecting negative adjustment. The right part of Table 5 shows significant positive correlations between, on the one hand, the individual mean levels and rates of change of oppositionality and, on the other hand, the number of selfreported antisocial behaviors and psychopathology. Girls with high mean levels of oppositionality and whose level of oppositionality increased were likely to report greater involvement in antisocial behaviors and to experience more mental health problems. The prediction accuracy was .50 for antisocial behaviors and .36 for psychopathology, corresponding to a large effect size and a medium effect size, respectively.

The results (Table 5) revealed a very consistent pattern for the prediction of outcomes obtained by parent and teacher reports. Interindividual differences in the development of oppositionality appeared to be negatively related to social skills and positively related to psychopathology. Individual mean levels of oppositionality were significantly and negatively related to parent-rated and teacher-rated social skills, with the exception of teacher-rated assertion. The rates of change of oppositionality were significantly and negatively related to teacher-rated social skills only. The results indicate that girls with high mean levels of oppositionality are more likely to be rated by their parents as low on all social skills. In addition, girls with high mean levels of oppositionality and whose level of oppositionality increased, were likely to be rated by their teachers as uncooperative and low on self-control. Teachers were also more likely to rate girls whose oppositionality increased over time as less assertive. The accuracy in the prediction of parent-rated social skills ranged from .18 for cooperation to .35 for self-control, corresponding to medium effect sizes. The accuracy in the prediction of teacher-rated social skills ranged from .24 for assertion to .44 for cooperation. These correlations correspond to medium effect sizes. 
Individual mean levels of oppositionality were significantly and positively related to parents' and teachers' judgments of child psychopathology. Girls who were more oppositional during early adolescence appeared to be at increased risk for mental health problems. Developmental changes in oppositionality were not related to parents' judgments, but rates of change of oppositionality were significantly and positively related to teachers' judgments of child psychopathology. This indicates that girls with high and increasing levels of oppositionality were at risk for mental health problems according to their teachers. The prediction accuracy of .32 for parents' judgments corresponds to a medium effect size. The correlation between predicted and observed teachers' judgments of .47 corresponds to a large effect size.

Table 6.

Comparison of the mean oppositionality scores (intercepts, slopes) and scores of children with poor academic achievement scores and those who reported involvement in substance use, property damage, and illegal acts

\begin{tabular}{|c|c|c|c|c|c|c|}
\hline \multirow[b]{2}{*}{ Outcome } & \multicolumn{2}{|c|}{$\%$} & \multicolumn{2}{|c|}{ Mean intercept } & \multicolumn{2}{|c|}{ Mean slope } \\
\hline & Boys & Girls & Boys & Girls & Boys & Girls \\
\hline Mean trajectory & & & 7.88 & 4.28 & .32 & .60 \\
\hline \multicolumn{7}{|l|}{ Academic Achievement } \\
\hline Lower $20^{\text {th }}$ percentile (national norms) & 15.5 & 21.8 & 9.89 & 5.39 & .64 & 1.16 \\
\hline \multicolumn{7}{|l|}{ Substance use } \\
\hline Alcohol only & 38.9 & 28.2 & 8.11 & 4.45 & .52 & .86 \\
\hline Alcohol and cigarettes & 15.2 & 9.1 & 10.16 & 5.93 & .33 & 1.41 \\
\hline \multicolumn{7}{|l|}{ Property damage } \\
\hline Purposely damaging something at home & 29.4 & 23.7 & 8.01 & 5.37 & .30 & 1.04 \\
\hline Purposely damaging something at school & 15.9 & 8.5 & 10.21 & 6.66 & .76 & 1.41 \\
\hline \multicolumn{7}{|l|}{ Illegal acts } \\
\hline Police contacts & 21.7 & 2.9 & 8.52 & 6.82 & .58 & 1.24 \\
\hline Shoplifting, something worth $<10$ Euro & 11.1 & 7.6 & 10.04 & 6.52 & .46 & 1.59 \\
\hline
\end{tabular}

Note. The antisocial behaviors listed are ranked in order of seriousness and represent a subset of the total list of acts.

Boys and girls. To substantiate these findings, Table 6 displays the mean level of oppositionality and mean rate of change for boys and girls with poor academic achievement scores (scores below the 20th percentile) and for boys and girls who reported different forms of substance use, property damage, or illegal acts. The table also displays the percentages of boys and girls who reported these antisocial acts. It can be seen that the trajectories of both the lowest-achieving boys and girls were higher and steeper than their mean developmental trajectories. However, girls with poor academic achievement scores appeared to have been on 
trajectories characterized by substantially lower mean levels of oppositionality, but steeper slopes than boys with similar academic achievement scores.

Table 6 substantiates the observed gender difference in antisocial behaviors by showing that girls less frequently reported involvement in antisocial activities than boys with the greatest differences found for the more serious antisocial behaviors (damaging something at school, police contacts, shoplifting). The table further illustrates and extends our findings on the predictive value of developmental trajectories of oppositionality with regard to antisocial behaviors. The trajectories of boys and girls who reported more serious antisocial behaviors were not only characterized by substantially higher mean levels of oppositionality and a steeper slope than the mean trajectories, but also by higher mean levels and steeper slopes than the mean trajectories for those who engaged in less serious acts. For girls, the difference in slopes is especially noteworthy. Trajectories of girls who had used alcohol and cigarettes or had damaged something at school appeared to be more than twice as steep as the mean trajectory. Although the very small percentages of girls reporting illegal acts do not allow comparisons, the same picture emerges here.

\section{Discussion}

The aim of the present study was to examine the longitudinal relationships between developmental trajectories of oppositionality during early adolescence and a diverse set of outcome variables measured at the transition from primary education to secondary education. Our study differs from other prediction studies in its focus on developmental changes of child-reported oppositionality and the inclusion of multiple outcomes reflecting both negative adjustment and positive adjustment. Furthermore, by including both boys and girls the present study is the first to provide insight into gender differences in the development of oppositionality and its link to later adjustment. An additional strength of the present study is that we gathered information on developmental outcomes from multiple informants.

\section{Oppositionality trajectories and developmental outcomes}

The results showed that boys on average become somewhat more oppositional during early adolescence. In addition, there appeared to be large individual differences in developmental trajectories of oppositionality. Interindividual differences in the mean level of oppositionality predicted all of the different outcomes except autonomy. Boys high on oppositionality were likely to do less well at school, to have low self-esteem, to perceive less social support, and to experience elevated mental health problems. In addition, both parents and teachers rated boys with high mean levels of oppositionality low on social skills and high on overall psychopathological symptomatology. Both the individual mean levels of oppositionality and rates of change were related to teacher-rated cooperation and self-reported antisocial behaviors. Boys who displayed both high and increasing levels of oppositionality 
were likely to report greater involvement in antisocial behaviors and to be identified by their teacher as uncooperative.

For girls, oppositionality also typically increased toward higher levels during early adolescence, and even more so than for boys. Again, there was substantial heterogeneity with respect to both the mean level of oppositionality and the rate of change. Interindividual differences in the development of oppositionality appeared to be related to all of the outcomes except social support from close friends. Girls who displayed high levels of oppositionality over the early adolescent years appeared to feel less autonomous. Furthermore, girls with high and increasing levels of oppositionality were likely to do less well at school, to have low selfesteem, to experience less support from their teacher and classmates, and to report more psychopathological symptoms and greater involvement in antisocial behaviors. In addition, both parents and teachers identified girls with high mean levels of oppositionality as low on social skills and at heightened psychiatric risk. Teachers also were more likely to rate girls with increasing levels of oppositionality as low on cooperation and self-control, and high on mental health problems. The implications and ramifications of these results will be discussed in the next sections.

\section{Developmental course and developmental significance}

Children, especially girls, typically show an increase in opposition during the last three years of elementary school, confirming earlier studies on the developmental course of externalizing problems during this period (Galambos et al., 2003; Wångby et al., 1999). The observed developmental patterns are in line with the notion that the terrible two's are followed by a second oppositional phase in early adolescence (Anthony, 1976) and the general public's perception that early adolescence is a period in which young adolescents increasingly frustrate their parents and teachers with their resistant and defiant behavior. In addition, the findings support the modified 'storm and stress' view of adolescence as proposed by Arnett (1999). In this view, increases in oppositionality during early adolescence are not seen as inevitable and universal, but it is recognized that many young adolescents become more willing to disagree with those in authority and that many parents perceive of early adolescence as a difficult time because of frequent (authority) conflicts with their child.

The current study not only offers an improved understanding of the developmental course of oppositionality during early adolescence, but also clearly shows the implications of oppositionality for adolescent's psychological, social and academic functioning at the end of elementary school. The pervasiveness of the detrimental effects of oppositionality on outcomes across domains and informants is striking. Children's self-reported oppositionality predicted negative developmental outcomes only, irrespective of whether those outcomes were academic achievement reports from the National Institute for Educational Measurement (CITO) or outcomes obtained by self-reports, parent reports, or teacher reports. The data support the risk hypothesis by showing that comparatively high levels and strong increases of 
oppositionality may have negative consequences and that oppositional interactions with parents, teachers, and peers can lead to a host of difficulties at the end of elementary school.

The data provide no support for the competence-promoting hypothesis, which assumes that oppositionality promotes the development of individuation and autonomy. On the contrary, oppositionality is associated with a heightened risk for self-esteem problems and, at least for girls, with less autonomous behavior. The findings rebut that opposition can be regarded as constructive behavior in mastering the developmental challenges of early adolescence. It appears that oppositionality has a quite disturbing effect on children's relationships and may be indicative of diminished social competence. Therefore, we argue that opposition should be distinguished from competent forms of negotiating one's independence and establishing a consolidated identity. Crockenberg and Litman (1990) investigated several ways in which 2-year-olds negotiate their independence by observing mother-child interactions. They concluded that oppositionality is a form of defiance, whereas self-assertion can be regarded as an appropriate manifestation of autonomy. In early adolescence, the child's increased (social-) cognitive capacities and emotion-regulation abilities may make a distinction between appropriate manifestations of autonomy and oppositionality even more appropriate.

Our findings strongly support such a distinction. Although oppositional children do not appear to seriously lag behind in the development of autonomy, they seem to have been unable to obtain their independence without losing support from significant others. Furthermore, our findings show that oppositionality is negatively related to assertion and selfcontrol, suggesting that oppositionality can to some extent be regarded the opposite of defending one's self-interest in a socially accepted way and that oppositionality is associated with self-regulation deficiencies. This indicates that opposition is indeed associated with diminished social competence, including poorer self-assertion and self-control skills, possibly resulting in a lack of social support, and as such should be distinguished from competencepromoting behaviors and age-appropriate manifestations of autonomy, such as parent-child discussion (Brody et al., 2002), self-assertion, and the use of negotiation strategies and persuasion (Kuczynski \& Hildebrandt, 1997).

\section{Gender differences}

Our results address developmental changes and their link to a variety of outcomes in boys and girls. Across this age period, oppositionality appeared to increase relatively more for girls than for boys, but it did not reach the level in boys. No support was found for gender differences in developmental outcomes of oppositionality. Oppositionality during the transition from preadolescence to early adolescence predicted only negative outcomes for both sexes and there was no support for sex-differentiated outcomes. Although it appears that the consequences of oppositionality do not differ between boys and girls, it is important to note that on average girls were substantially less oppositional and that there was a substantial 
gender difference in one of the outcomes, i.e. girls reported far less antisocial behaviors. By comparing boys and girls who reported different forms of antisocial behaviors and the lowestachieving boys and girls on the academic achievement test, gender differences became more clear. Both the lowest-achieving girls as well as those girls who were involved in antisocial acts had been less oppositional, but had experienced more accelerated increases in oppositionality than boys with the same academic achievement scores or boys who reported involvement in the same antisocial behaviors. In addition, it appeared that girls were less frequently involved in the more serious forms of antisocial behavior and behaviors reflecting illegal activities. So although girls who are more oppositional than their same-sex peers are at risk for negative outcomes, boys' standards with regard to oppositionality and antisocial behaviors do not appear to apply to girls, not even to those most at risk.

The results further suggest gender differences in the process responsible for the link between early opposition and later adjustment problems. The predictive power of developmental changes in oppositionality appeared to be substantially larger for girls. For boys, strong increases in oppositionality predicted a lack of social skills in the domain of cooperation and greater involvement in antisocial behaviors. For girls, increases predicted poor academic achievement, a lack of social support from teachers and classmates, less social skills, greater involvement in antisocial behaviors, and increased risk for psychopathology.

This raises the question why the detrimental effects associated with developmental trajectories towards higher levels of opposition were particularly strong for girls. The sharp contrast between girls' behavior in childhood and that at the onset of adolescence (as noted in the introduction) may provide a possible explanation. Possibly, the rather rapid changes in girls (from nice girls to oppositional teenagers) evoke intense negative reactions from others. Some support for this explanation can be inferred from our results. In line with interpersonal accounts of oppositionality (Alexander \& Pugh, 1996; Mones, 1998), girls who became more oppositional were likely to report diminished support from their teacher and, to a lesser extent, lowered support from their classmates. These findings are in line with the notion that support erosion effects are more likely to occur in relationships for which the proscriptions about withdrawing social support are not so strong (Stice, Ragan, \& Randall, 2004). Given that adolescents' relationships with teachers are largely characterized by formal and social rules (Leman \& Duveen, 1999) and not much by closeness (as it is with parents and close friends), it is perhaps not surprising that teachers are the first to respond in a negative way to oppositional girls, e.g. they may withdraw their support. In the Netherlands, it is common that teachers change every year supporting the direction of the effect. In addition, support erosion may indicate a severe disruption in the relationship with the teacher, which may in turn lead to poor academic achievement (see Ledingham, 1999), thereby explaining the observed link between changes in oppositionality and later academic achievement.

Alternatively, one could argue that the observed difference is an indication of a developmental lag between boys and girls. Given the fact that girls begin puberty earlier than 
boys and the expected impact of puberty on the development of oppositionality, individual differences in developmental change may be the greatest during early adolescence for girls, whereas they may first come to the fore in middle adolescence for boys. The limited timeframe of the current study hinders a test of this line of reasoning. However, our findings seem to contradict that individual differences in the rate of change of oppositionality will first emerge during middle adolescence for boys. First, although girls seemed to increase relatively more, there was also substantial individual variability in the rate of change for boys. The finding that these interindividual differences in the rate of change were associated with elevated levels of antisocial behavior and a lack of cooperation (as rated by teachers) further indicates that meaningful individual differences in developmental change of boys' oppositionality can be observed during early adolescence and are not restricted to later in their development.

\section{Predictive value of developmental change}

An important conclusion to be drawn from the present study is that changes in oppositionality during early adolescence have predictive value over and above the child's mean level of oppositionality, thereby confirming that the developmental process is a crucial factor in linking early experiences and behaviors to later functioning (Haselager, Cillesen, Van Lieshout, Riksen-Walraven, \& Hartup, 2002). We offered in the introduction potential psychological mechanisms that can account for the relation between changes (increases and decreases) in oppositionality and the adolescents' social, academic and psychological functioning at the end of elementary school. However, future research is needed to explicate and substantiate these proposed mechanisms.

As far as we know, the current study is the first to present empirical evidence that adolescents who have progressed along an early oppositionality pathway are at increased risk for involvement in antisocial behaviors at the end of elementary school. The observed developmental progression from everyday oppositional behaviors to antisocial behavior confirms existing developmental theories of antisocial behavior that have posited a path toward antisocial behavior characterized by an early onset and developmental continuity (Loeber \& Stouthamer-Loeber, 1998; Moffitt, 1993; Patterson, DeBaryshe, \& Ramsey, 1989). There appears to be consensus that age of onset and persistence are key features in describing different types of developmental trajectories toward later antisocial behavior (Vermeiren, 2003). The present study indicates that not only persistence of a high level of oppositionality, but also a strong increase of oppositionality at late school-age puts children at risk for developing antisocial behavior in early adolescence.

\section{Limitations and future research directions}

There are several limitations of our study that should be acknowledged. The last measurement occasion of the longitudinal data (predictor) coincided with the assessment of 
the outcome variables. As a result, the predictive accuracy may have been overestimated. However, research with short follow-up periods does not necessarily lead to high predictive accuracy. Although we are unaware of studies that have examined the effect of duration of follow-up on predictive accuracy, a review of 13 studies with follow-up periods varying from 1 to 7 years revealed that for all of these studies the predictive accuracy of externalizing behaviors in normal populations was modest at best (Bennett, Lipman, Racine, \& Offord, 1998). In addition, it is our perspective that most concerns about short follow-up periods are inspired by the fact that the stability of behavior over short periods of time is greater than over longer periods (Olweus, 1979). Our results pertain to the predictive value of the level of oppositionality averaged across a 3-year period and developmental changes in oppositionality during this period rather than the stability over time of oppositionality. Therefore, we feel confident that our results reveal important aspects of the developmental significance of oppositionality during early adolescence, although we acknowledge that a follow-up at secondary school or at young adulthood would be a very welcome addition to the current results.

Another limitation may be the choice of outcomes. Although we included a wide variety of outcomes, one could argue that we may have missed important ones. For example, it would be desirable to complete the current range of outcomes in future research with official (conviction) records of juvenile delinquency in order to have an objective measure of antisocial behaviors. In addition, it seems that future research could benefit from differentiating between various forms of antisocial behavior and by including measures that may be especially relevant to girls (e.g., engaging in risky sexual behaviors; Henning-Stout, 1998). A final limitation involves the effect of attrition and nonresponse on the outcome measures. Although we feel we have adequately addressed this potential problem by estimating the resulting bias, it is possible that some may object to this approach or do not consider the estimated bias to be negligible.

Despite the possible shortcomings of this study, we have demonstrated the importance of developmental changes in oppositionality during early adolescence in predicting a wide variety of developmental outcomes. It appears that oppositionality and increases in oppositionality with age can put young adolescents at risk for serious disadvantages at the end of elementary school. Although the limited time-frame of the current study precludes a longterm perspective, it seems that these disadvantages at a time when entry into a new school system requires a degree of psychological maturity can have very detrimental effects for young adolescents' future development. In this respect, observed links between poor school adjustment at age 14 and long-term unemployment (Kokko \& Pulkkinen, 2000) and between a lack of parental support in childhood and depressive symptoms and chronic conditions in adulthood (Shaw, Krause, Chatters, Connell, \& Ingersoll-Dayton, 2004) do not help to make us any more optimistic of the long-term effects of oppositionality. 


\section{Implications}

The results from our study have some general methodological implications as well as implications for interventions and clinical practice. The finding that developmental trajectories of oppositionality accounted for substantial variation in most of the outcomes represents a departure from the notion that the prediction of most adolescent and adult outcomes from childhood disruptive behavior is only modest to moderate (Hinshaw, 2002). Especially the medium to large effect sizes for the prediction of academic achievement, teacher-rated cooperation skills, and adult ratings of children's mental health problems are noteworthy, because they indicate high prediction accuracy that can not be attributed to shared method variance. The high predictive value of the development of oppositionality for a variety of developmental outcomes seems rather surprising given that we relied on self-report data for the estimation of developmental trajectories. Previous research has raised doubt concerning the predictive validity of self-reported oppositionality. Loeber, Green, Lahey and Stouthamer-Loeber (1991) found that whereas adult reports were related to several negative outcomes, self-reported oppositionality was related to only one of these outcomes. There are two features of the instrument used to assess oppositionality in this study contributing in a positive way to the predictive value of self-reported oppositionality. First, the descriptions of everyday interactions with parents, teachers, and peers in the ASO probably provide more valid responses than asking children about the frequency and intensity of clinical symptoms. Second, the facet design of the instrument certified a systematic sampling of children's emotional and behavioral responses across different situations. As a result, the total oppositionality score on the ASO reflects a compilation of children's oppositionality at home, at school, and in situations with peers.

Although the properties our research instruments may have resulted in more precise measurements, our very good and thereby atypical prediction results can possibly best be explained by the fact that most previous studies have emphasized stability in disruptive behaviors at the cost of developmental changes (Flory, Lynam, Milich, Leukefeld, \& Clayton, 2004). Approaches commonly used for prediction have predominantly involved correlations between two time-lagged measurement occasions and regression analyses of the outcomes on prior measurements. There has been relatively little attention to developmental trajectories connecting early behaviors to later outcomes. Results of our prediction model linking individual developmental trajectories to later outcomes demonstrate that information from repeated assessments can provide a stronger basis for prediction than the child's functioning at a single point in time (Mahoney \& Bergman, 2002; Schaeffer et al., 2003). In addition, we showed that models that take developmental changes explicitly into account provide a more useful and more insightful tool for prediction purposes.

The predictive value of developmental changes has also implications for the implementation of interventions. In order to successfully intervene, one should first be able to identify cases for whom intervention is a high priority with some precision. This is usually 
done by conducting a screening. A recent study of the effectiveness of early screening for externalizing problems indicates that screening at first grade was equally effective as screening at both kindergarten and first grade (Hill, Lochman, Coie, Greenberg, and Conduct Problems Prevention Research Group, in press), suggesting that screening at multiple time points does not increase the effectiveness. Our results seem to contrast with the conclusion that screening based on a single assessment is equally effective as a screening based on repeated assessments. The findings clearly suggest that tracking children's behavior problems across elementary school (e.g. in a school-based program or monitoring system) can improve screenings by identifying all children at risk, i.e. not only those who are on a trajectory characterized by persistently high levels of oppositionality, but also those who are on a trajectory of increasing problems. In line with Schaeffer, Petras, Ialongo, and Kellam (2003), we argue that screening for youths at risk should therefore involve multiple time points. In addition, our results suggest that the success of intervention programs could well lie in the prevention of increases in oppositionality rather than in reducing these behaviors (c.f. Catalano et al., 2003).

Finally, our results have implications for categorical approaches to the assessment of oppositionality. First, the present study clearly indicates that oppositional problems should be taken seriously. Everyday oppositional interactions with parents, teachers, or peers may often involve conflicts over mundane matters or disputes over seemingly irrelevant social conventions. Nevertheless, if such mildly disturbing interchanges are of a repetitive nature, our findings suggest that they may have severe consequences. Second, the predictive validity of self-reported oppositionality has implications for the assessment of oppositionality. The present study strongly suggests that the child itself can make a significant contribution to the assessment. And finally, our results appear to imply that the assessment of oppositionality may be more complicated than sometimes portrayed. In order to diagnose clinically relevant levels of oppositionality, clinicians face the difficult task of determining whether the child's oppositionality is age-appropriate or not. This task seems to be complicated by the observed developmental changes in oppositionality. A recent deterioration in the child's home or school behavior may be a major reason why parents and teachers seek mental health care. It therefore seems that clinicians need to figure out whether the child has been referred to the clinic because of the expected and typical increase in oppositionality during early adolescence or a more serious upsurge in the child's rebelliousness and resistance and this may be especially true for girls. In this respect, information on the preceding developmental course of oppositionality may be very useful. Although the child's developmental history is generally acknowledged as an important part of the assessment of oppositionality, most clinicians tend to focus on early temperament, attachment history or family history (c.f. Greene \& Doyle, 1999). We argue that obtaining information on the developmental history of the child's oppositional problems, including recent changes, should be standard practice. 


\section{CHAPTER 7}

General Discussion 
The main aim of this thesis was to gain a better understanding of the development of oppositionality. For this purpose, five empirical studies were conducted, of which the first three focused on the assessment of oppositionality and the last two focused on the development of oppositionality and its associated outcomes. This chapter aims to integrate the results from these studies. For this purpose, the separate studies will be summarized and discussed with regard to several key issues: (1) the (developmental) significance of oppositionality during early adolescence; (2) the value of self-reported oppositionality; (3) situational specificity; (4) and gender differences. These issues can be considered as common themes that run throughout multiple chapters of this thesis. In addition, this chapter includes some methodological considerations, discusses the clinical implications of our results, and provides recommendations for future research efforts based on the strengths and limitations of our studies.

\section{The (developmental) significance of oppositionality during early adolescence}

Oppositionality can be seen as a form of problem behavior as well as a "normal" developmental phenomenon reflecting children's changing conceptions of authority and increasing sense of autonomy during early adolescence (Braungart-Rieker, Garwood, \& Stifter, 1997; Kuczynski \& Hildebrandt, 1997). Chapter 6 takes these two opposing views on oppositionality as the point of departure. The chapter takes a developmental approach to empirically evaluate the significance of oppositionality during early adolescence. Two competing hypotheses were formulated: the risk-hypothesis and the competence-promoting hypothesis. The risk-hypothesis assumes that oppositionality has detrimental effects only. According to the competence-promoting hypothesis, oppositionality will be related to positive outcomes. It is expected that oppositional children will turn out to be more autonomous, selfconfident, and self-assertive adolescents.

The results show that comparatively high levels and strong increases of oppositionality were not related to higher levels of autonomy, but in contrast were associated with a heightened risk for poor academic achievement, a lack of social support, poor social skills, antisocial behaviors, and child psychopathology. These results clearly support the riskhypothesis. The findings rebut that opposition can be regarded as constructive behavior in mastering the developmental challenges of early adolescence. Therefore, we argue that oppositionality should be distinguished from competence-promoting behaviors and ageappropriate manifestations of autonomy.

The studies in this thesis further point to the significance of the interpersonal component in oppositionality (Mones, 1998). Anthony (1976) already stated that: “... it takes two to produce oppositional behavior..."(p. 3). The conclusion that oppositionality does not occur within the vacuum of the individual (Alexander \& Pugh, 1996), but within relationships with parents, teachers, and peers, is supported by the observed situational specificity in oppositionality (discussed in a separate section) and analyses of the relations between 
oppositionality in different interpersonal situations and parent and teacher ratings of criterion behaviors (chapter 3). The latter underlined the importance of distinguishing between oppositionality in interactions with parents, teachers, and peers. It was found that distinguishing between oppositionality in different situations provides more precise research findings with regard to relations with external variables. Oppositionality in situations with teachers appeared to be more strongly related to conduct problems than oppositionality in situations with parents or with peers.

The observed differential relations between oppositionality and conduct problems across situations suggest that individual differences in oppositionality are more easily revealed at school than at home, possibly indicating that the school context triggers oppositional responses to a greater extent than the family context. In addition, researchers have emphasized the centrality of the school context for children's development (Maughan, Rower, Messer, Goodman, \& Meltzer, 2004). It could therefore be valuable to examine the impact of school-specific oppositionality. It may be that increases in oppositionality in this particular situation have particularly strong detrimental effects for children adjustment.

In sum, our results lead us to conclude that two important elements should be included in a definition of oppositionality. First, oppositionality reflects a tendency to resist and break social rules rather than an expression of individuality or autonomy. The core element of oppositionality is a willful contrariness (Redl, 1976). Second, oppositionality is of an interactive nature (Kalb \& Loeber, 2003), requiring somebody in authority or a social rule (implicitly or explicitly formulated).

\section{The value of self-reported oppositionality}

All of the studies in this thesis used children's self-reported oppositionality as obtained by the ASO. This contrasts with most studies on oppositionality that have typically relied more heavily on information provided by informants other than the child (Johnston \& Murray, 2003). It is likely that most researchers have more confidence in adult informants because of possible contaminating factors in self-reports (e.g. social desirability and underreporting). Because of these doubts on the validity of self-reports, chapter 2 and 3 investigated to what extent oppositionality can be validly assessed by using self-reports. The results reported in these chapters support the reliability of the ASO, its structural validity, and criterion-related validity. In addition, the results reported in chapter 6 provide strong support for the predictive validity of self-reported oppositionality as obtained by the ASO. Both the adolescent's mean level of self-reported oppositionality as well as the rate of change of self-reported oppositionality appeared to have predictive value for a wide range of outcomes. Taken together, we have found considerable support for the validity of children's self-reports in the assessment of oppositionality, suggesting that self-reports have undeservedly been neglected in the assessment of children's behavior. 
Some of our findings suggest that self-reported oppositionality cannot only offer valid information, but also unique information. First, our results indicate that oppositionality is best conceptualized as a combination of emotional and behavioral responses (chapter 2). Although there are obviously objective aspects of the child's oppositionality (e.g., the frequency of conflicts with authority figures or rule violations), some events at school or at home are likely to trigger subjective responses, i.e. oppositional feelings (e.g. frustration in response to restrictions and prohibitions), without directly leading to oppositional behavior. More or less 'objective' methods, such as obtaining ratings by independent observers, may fail to identify these non-observable oppositional feelings. In addition, these methods may have trouble identifying certain behaviors as acts of opposition because of their ambiguity. For example, simply ignoring a request may be an indication of passive noncompliance (Kuczynski \& Kochanska, 1990), but can also reflect the child's failure to hear or comprehend the request. Therefore, we argue that events can be better characterized as oppositional interactions by the meaning the child assigns to them. Consistent with the importance of the perceived or interpretive social context in the development and prediction of psychopathology (Boyce et al., 1998), assessing the meaning of characteristic social interactions to the individual child who is likely to experience such interactions may provide unique and valuable information.

Second, the results reported in chapter 3 revealed unique relations of oppositionality at home and at school with parent and teacher ratings of criterion behaviors. The findings underscore the situational specificity of oppositionality and indicate that the limited access of adult informants to situations outside the context in which they naturally observe children may also seriously limit the value of their reports. The findings support the assumption that children themselves are able to provide unique information about interactions with individuals across a broad range of situations and point to the possibility of developing psychometrically sound instruments that take full advantage of the fact that the child is the only "insider" in a range of relationships (Furman \& Buhrmester, 1985).

Although self-reports seem able to provide unique information, we recognize that psychological assessment cannot be based on the information provided by self-reports alone. In addition, it should be noted that the present thesis did not examine whether self-reported oppositionality adds information to the assessment process that improves the outcome, i.e. the incremental validity of the ASO (Johnston \& Murray, 2003). This is a critical question that calls for future research on the contribution of child self-reports and adult reports to the prediction of objective criteria that are not confounded with the predictors (e.g., school records of the number of times punished or expelled and academic achievement scores (see chapter 6)).

\section{Situational specificity}

The present thesis yielded several findings that point to the existence of situationalspecificity in oppositionality. The results reported in chapter 2 lead to the conclusion that a 
substantial part of the child's opposition is determined by the specific characteristics of situations with parents, teachers, and peers. The situational specificity revealed in this crosssectional study was again observed in our longitudinal analyses reported in chapter 5 . The findings revealed that oppositionality only increased in situations with parents and teachers, and not in situations with peers. In addition, it appeared that during the early adolescent years a substantial proportion of the individual variability in developmental trajectories of oppositionality could be attributed to situational differences.

The topic of situational specificity has been addressed before. An extensive metaanalysis of cross-informants correlations by Achenbach, McConaughy, and Howell (1987) has shown that there is little agreement among informants on children's behavior problems. Similarly, Webster-Stratton (1990) found that teachers reported no excessive problem behaviors for $61 \%$ of a total of 77 children who were reported to be in the clinical range on noncompliance, aggression and oppositionality by their parents. Finally, longitudinal studies have also addressed situational differences in children's development by using both parent and teacher reports (Chan et al., 2000; Keiley et al., 2000). The results from these studies revealed differences between settings in both the direction of change as well as interindividual differences in intraindividual change. The findings of these studies suggest that children exhibit different levels of oppositionality in different situations and that children's development varies across situations. However, the observed differences can also be ascribed to differences in the perceptions of informants. In the design of these studies situational effects and informant effects were inevitably confounded. Parents observed children at home and teachers observed children at school.

Our studies relied on the information of the same 'observers' across different situations, i.e. the children. The observed situational-specificity in our studies is consistent with the conclusion by Achenbach et al. (1987) that the lack of inter-informant agreement between parents and teachers can be attributed to real differences in the child's behavior across different situations. The results reported in chapter 4 strengthen this conclusion by showing that informant disagreement on children's conduct problems is partly explained by situational differences in the child's self-reported oppositionality.

Taken together, these findings strongly suggest that situational demands affect oppositionality. This raises the question in what respect these situations differ? With regard to oppositionality, the most important differences were noted in this thesis. First, situations with parents, teachers, and peers differ in the characteristics of the relationships involved in each of the different situations (Chapter 2). The parent-child relationship can be typified as affectionate, secure, non-voluntary and enduring (Bigelow et al., 1996), whereas relationships with teachers are less affectionate, non-voluntary and only partly enduring. Second, each of these situations is characterized by different grades of elasticity or flexibility (Henker \& Whalen, 1999). Parents apply rules in a rather flexible way and adapt their behavior to their child's needs, whereas teachers are bound to the rules and constraints of the school. 
Consequently, teachers have far less possibilities to adapt to individual differences within the classroom. In addition, the school context offers children a well-defined social-organizational system with a unique emphasis on both performance and social rules (Henker \& Whalen, 1999). Within the school system, teachers hold an official authority position (Laupa et al., 1995) and classrooms constitute a peer audience.

We also offered several potential psychological mechanisms that may explain why the aforementioned situational differences give rise to situational-specificity in oppositionality. These explanations were largely inspired by reactance theory (Brehm, 1981). This theory hypothesizes that characteristics of the social agent, the force of the threat to the child's perceived behavioral freedom, and the importance of the freedom affect the magnitude of reactance (oppositionality). Future research is needed to test whether oppositional interactions can successfully be interpreted in terms of reactance theory. In addition, increased attention to the situational variation in children's behavior could further our understanding of which situational and relational characteristics and underlying mechanisms give rise to it. In this respect, it is noteworthy that we have recently applied the concepts of authority, reciprocity, and closeness to relationships with parents, friends, and teachers and have found that these relationship characteristics were meaningfully related to oppositionality within situations with parents, teachers, and peers (Mijné, 2003). It was found that children who experienced closeness and reciprocity in their relationships appeared to be less oppositional.

An important implication of the observed situational specificity is that measures that do not provide full recognition of the situational variability in the child's behavior can obscure important information about the pattern of children's responses across social contexts. In this respect, our studies connect to two studies by Wright and Zakriski (2001, 2003), in which they showed that groups of externalizing children were characterized by considerable within-group heterogeneity in both their reactions to events and the responses those reactions in turn evoke from others. In line with these authors, we argue that traditional approaches to assessment might wrongfully focus our attention on the frequency and intensity of children's overall behavioral output and fail to inform us on the relationship between children's responses and the situations in which they occur.

\section{Gender differences}

Because of a primary focus on boys in research on disruptive behaviors, little is known about gender differences in oppositionality. Our studies were based on relatively large representative general population samples that were fairly evenly divided according to gender. They incorporate gender comparisons on the level of oppositionality, the manifestation of oppositionality across situations, and the correlates of oppositionality. In addition, the longitudinal studies provide information on gender differences in the developmental trajectories of oppositionality during early adolescence and their consequences. 
From the results reported in chapter 2, it is clear that boys are more oppositional than girls. This is consistent with the supposed interpersonal vulnerability of girls. We speculated that girls may be more sensitive to interpersonal concerns and therefore more inclined to refrain from behavior that could affect relationships negatively. A second explanation for the observed gender difference may be that boys are more vigilant toward autonomy threats and are more easily frustrated by the actions of others. Although gender differences in interpersonal vulnerability (Leadbeater et al., 1999) and threat perception (Rutter, Caspi, \& Moffitt, 2003) have been noted in the literature, there is a need to test whether these differences actually can account for the observed gender differences in oppositionality.

The results reported in chapter 2 showed both differences and similarities in the manifestation of oppositionality. The results revealed gender differences in the level of oppositionality across situations. It appears that gender differences in oppositionality are most pronounced in situations with peers, somewhat less pronounced at school, and virtually absent at home. Boys and girls did not show different situational patterns of oppositionality. Both sexes were more oppositional at home than in interactions with teachers or peers. In addition, the findings indicate that girls are remarkably similar to boys in their responses to their parents. Together, these findings show that failure to attend to all of the situations in which oppositionality can occur can lead to incorrect conclusions regarding gender differences. Boys and girls appear to be very similar in the way to they respond to their parents and in their pattern of responses across situations.

We uncovered few gender differences in the correlates and consequences of oppositionality. Chapter 3 explored gender differences in the relation between oppositionality and parent and teacher ratings of children's psychopathology and social functioning. The results revealed that boys and girls are very similar with regard to the pattern of associated behaviors. For both sexes, oppositionality appeared to be positively related to conduct problems and hyperactivity, and negatively related to pro-social behavior. Chapter 6 explored gender differences in the outcomes linked to developmental trajectories of oppositionality. The results revealed no gender differences in the types of adjustment problems associated with high and increasing trajectories of oppositionality. Together, these findings seem to suggest that oppositional responses in interactions with parents, teachers, and peers reflect the same underlying liability for boys and girls. It is concluded that oppositionality is an equally important indicator of adjustment problems in both boys and girls.

All in all, gender similarities in oppositionality seem to be more common than gender differences. In addition, previous longitudinal studies have shown that developmental changes in oppositionality are the same for boys and girls (Bongers et al., 2004; Lahey et al., 2000), suggesting that no gender-specific factors are involved in the development of oppositionality. However, two findings in our longitudinal studies (chapter 5 and 6) contradict this conclusion by pointing to gender-specific processes in the development of oppositionality. First, girls appeared to catch up with boys in the level of oppositionality during early adolescence. 
Second, the detrimental effects of increases in oppositionality during early adolescence were particularly strong for girls.

On the basis of the latter two findings, we argue that it may be worthwhile to focus on gender differences in the development of oppositionality during the preadolescent to early adolescent years. Researchers have pointed out that changing gender differences provide "a window of opportunity for gaining insight about the aetiology of a particular disorder" (Rutter, Caspi, \& Moffitt, 2003, p. 1095). In addition, focusing on gender differences in the development of oppositionality and in the consequences that accrue can provide important clues for improving developmental theories of antisocial behavior. It is generally assumed that existing models for boys apply equally well to the development of antisocial behavior in girls, but our results call this assumption into question. The particularly strong link between increases in girls' oppositionality and various types of maladjustment, including antisocial behavior, suggests that girls may change rather rapidly from nice girls to oppositional teenagers (Crick \& Zahn-Waxler, 2003) and that this sudden turnover is related to their involvement in antisocial activities. Therefore, along with Silverthorn and Frick (1999), we propose that alternative models specific to the development of antisocial behaviors in girls are needed. In sum, we believe that future research on gender differences in the development of oppositionality could help to clarify the causal mechanisms specific to the development of girls' adjustment problems.

\section{Clinical implications}

Although all of the chapters in this thesis lack a clear clinical perspective, several findings provide useful suggestions for diagnostic processes in clinical practice. First, our results suggest that clinicians need to take into account situational variation in children's oppositionality and that we may need diagnostic criteria that differentiate between children who show oppositional symptoms at home, at school or in multiple settings.

One could argue that situational differences in oppositionality are irrelevant in the assessment of psychological disorder, because the basic concept of disorder is the notion that the problem is in the person (Wakefield, 1993). Although the concept of disorder and its operational criteria may stress the idea that behavior problems occur within the vacuum of the individual rather than in interactions with significant others and within different contexts (Alexander \& Pugh, 1996), most clinicians intuitively realize that serious clinical problems are the result of various different forces (Denton, 1996). So, the DSM-IV may be biased toward the individual, whereas those who use this diagnostic system may be more open to the importance of environmental influences and the role of children's relationships with significant others.

Applied to oppositionality, the problem can be phrased as 'oppositional children' or 'oppositional behavior' in order to emphasize that neglecting the role of parents, teachers, and peers in the assessment of oppositionality can incorrectly lead to the identification of 
oppositional children. As Wakefield (1993) noted in his critique of the operational criteria for disorder, a child who meets the DSM-criteria for ODD may formally be an oppositional child. However, if the child's oppositionality is clearly a reaction to an over-restrictive or abusive environment, clinicians undoubtedly will not regard this behavior as dysfunctional. They will adjust their diagnosis even if no guidelines are provided in the DSM. Hsieh and Kirk (2003) nicely illustrated this. By using systematically manipulated vignettes, they showed that psychiatrists' judgments of the presence of mental disorder were significantly affected by the social context (e.g. living in a disadvantaged neighborhood), indicating that judgements are not made solely on the basis of DSM's operational criteria. By adjusting DSM-IV based diagnoses to their intuitions about the disorder, clinicians technically violate formal diagnostic rules (Wakefield, 1993). Therefore, it seems that standard assessment procedures should be refined. Increased attention to the situational aspects of oppositionality in standard assessment procedures can help clinicians to recognize the relationship between the behaviors and the situations in which they occur, leading to a better identification of potential relational and environmental factors in the child's oppositionality. Situation-specific oppositionality may point to relational difficulties or family dysfunction, whereas cross-situational consistency may point to compromised skills in the domains of emotion regulation or problem solving. Such information is of direct relevance to many aspects of clinical practice, such as treatment formulation.

A second clinical implication is that the assessment of oppositionality can benefit from including child self-reports. The validity of self-reported oppositionality strongly suggests that the child itself can make a significant contribution to the assessment of oppositionality. By means of the ASO, children can provide the clinician with direct information on the overall level of oppositionality as well as more detailed information about the situational aspects of the child's oppositionality. We consequently believe that adding the richness of information provided by the ASO could improve the diagnosis of ODD.

Self-reports may also be useful in solving the problem of how to deal with discrepant information from different informants. Clinical practice guidelines recommend that one should investigate whether discrepancies between informants reflect true situational differences (Achenbach, 1993), but provide the clinician with little clues on how to disentangle discrepancies due to different perspectives from discrepancies due to situational differences. Our results suggest that self-report instruments like the ASO might provide the clinician with the information needed, especially for externalizing problems. If the situational differences in children's responses across different situations are largely consistent with the discrepancies in reports by different adult informants, this strongly points in the direction of situational-specificity in the child's behavior. Although this seems to be a promising application of child self-reports, further research is needed.

Finally, our findings with regard to the development of oppositionality and outcomes linked to (deviant) developmental trajectories of oppositionality could help to define more 
precisely what is meant by clinically relevant levels of oppositionality. In order to diagnose clinically relevant levels of oppositionality, clinicians face two difficult tasks for which the DSM-IV offers little guidelines: determining whether the child's oppositionality is ageappropriate or not and determining clinically significant impairment. The first task would be lightened by the creation of a developmentally sensitive classification system. It has been suggested that the next DSM manual should incorporate a developmental framework for psychopathology (Widiger \& Clark, 2000). By providing basic descriptive data on the developmental course of oppositionality during early adolescence, our longitudinal findings may provide useful information for the development of this new diagnostic system with respect to ODD.

The second task is to determine clinically significant impairment. Chapter 6 provides important information in this respect by revealing the relation between oppositionality and levels of distress and impairment in the child's academic, social, and psychological functioning. Comparatively high levels and strong increases of oppositionality appear to have severe consequences for the quality of children's relationships, psychological functioning, and their academic achievement. The pervasiveness of the detrimental effects of oppositionality on outcomes across domains indicates that clinically significant impairment should be determined by taking a broad perspective. However, the correlational nature of this research makes that the directionality of effects is not certain. So although it may be that oppositionality leads to negative outcomes, one could also argue that many of these negative outcomes are in fact risk factors for oppositionality and developmental changes in oppositionality. For example, poor academic achievement could lead to frustration and alienation that eventually result in oppositional problems (Ledingham, 1999). Because the direction of effects has important implications for the focus of treatment, future studies should address the issue of causality by examining the dynamic relationship between oppositionality and children's adjustment.

\section{Methodological considerations}

Increased attention to the problem of how to assess intraindividual change has resulted in the widespread application of multilevel modeling to longitudinal data (Hertzog \& Nesselroade, 2003). This thesis has benefited from relatively new applications of the multilevel model that are not yet in widespread use. To our knowledge, chapter 5 is the first to apply a multivariate multilevel model to repeated measures of multiple indicators of the same construct, i.e. oppositionality in situations with parents, teachers, and peers. By simultaneously estimating developmental trajectories for oppositionality in three different situations, we were able to decompose interindividual differences in intraindividual change into generalized oppositionality and situation-specific oppositionality. The results strongly suggest that the process of oppositionality development depends on the situation in which it occurs. 
Chapter 6 presents another relatively new application of multilevel modeling. This chapter reports on one of the first studies to apply the multilevel model for longitudinal prediction to psychological data (Hoeksma \& Knol, 2001). The multilevel prediction model takes developmental changes explicitly into account. Our findings show that this model can provide researchers with a more useful and more insightful tool for prediction purposes.

More generally, our longitudinal studies have benefited considerably by carefully choosing our research design. More specifically, we choose to assess oppositionality twice a year over a 3-year period spanning the early adolescence years. Multiple measurement occasions enable a test of different change functions (linear or quadratic) and more accurate estimation of developmental trajectories. The high predictive accuracy of developmental trajectories of oppositionality (chapter 6) illustrates that focusing on a theoretically interesting developmental period and obtaining frequent measurements with short intervals during this period is likely to pay off.

Chapter 4 applied a methodological approach that simultaneously estimates the separate effects of the situational specificity of the child's behavior and informant characteristics. The methodological approach taken in this chapter is unique in that it provides an opportunity to disentangle the effects due to situational factors and informant factors. The method involved explaining source effects in informants' ratings on the basis of children's self-reports of situation-specific behavior and informant characteristics. Although Bollen and Paxton (1998) already discussed the possibility of investigating the determinants of source effects in subjective measures, we know of no studies that have followed up their suggestions and have tried to explain source effects in multiple informant data.

\section{Limitations}

The present thesis should be considered in light of its limitations. First, the assumption that oppositionality can be conceived as a continuum was not empirically tested. Nevertheless, several statistical techniques (e.g. taxometrics; Ruscio \& Ruscio, 2004) are available that can reveal whether a construct is indeed best represented by a continuum. So, although all findings together do not contradict the assumption of an underlying continuum, future research could employ these statistical techniques to test this assumption.

The validity of the assumption that the ASO measures the same underlying construct across the early adolescent years was also not empirically evaluated. In our view, the assessment of construct invariance (see Meredith and Horn, 2001) becomes a more critical issue if a broad age period is being studied. In addition, our longitudinal studies of developmental changes in oppositionality during early adolescence were preceded by an explicit evaluation of the structure of oppositionality during this period (chapter 2). Nevertheless, empirical support for the measurement equivalence and construct invariance of oppositionality would strengthen the conclusions from our longitudinal studies. 
Our longitudinal analyses were based on the assumption that a developmental trajectory reflects "a continuous and individual-specific underlying process" (Curran \& Willougby, 2003, p. 582). Again, the question is whether this is an appropriate assumption. Other longitudinal studies have used a typological approach to understand developmental change. The typological approach empirically identifies subgroups of children following common developmental trajectories and is based on the assumption that qualitative differences in patterns of developmental change exist. Both approaches have their strengths and weaknesses and it may be impossible to determine which approach corresponds to reality (Bauer \& Curran, 2003b).

However, for two reasons we preferred to model interindividual differences in the development of oppositionality as quantitative differences in the underlying function of the individual developmental trajectories. First, grouping the total range of developmental trajectories may lead to a loss of information about individual differences because the typological approach masks differences in developmental patterns among children within a supposedly homogenous subgroup. In addition, children within a subgroup may share a similar developmental pattern, but their characteristics (e.g. level of impulsivity) may vary. Because of the possible loss of information and the lack of strong evidence to support the assumption that children following a common developmental trajectory represent a 'true' subgroup of individuals, it may be more appropriate to consider the total range of possible changes in oppositionality, i.e. treating the interindividual variation in developmental trajectories as continuous (Hertzog \& Nesselroade, 2003). Second, focusing on quantitative differences in the developmental course of oppositionality rather than qualitative differences may also be preferable given that non-normal distributions of the repeated measures can produce evidence of subgroups even if the population truly consists of a single group of individuals (Bauer \& Curran, 2003a).

A final limitation pertains to our study of the link between developmental trajectories of oppositionality and positive and negative developmental outcomes (chapter 6). The research design of our longitudinal study made that we were unable to control for initial levels of children' adjustment. This limitation prevented us from making stronger claims concerning the effect of oppositionality on children's adjustment. Ideally, children's academic, social, and psychological functioning had not only been assessed at the last measurement occasion, but also at the first measurement occasion or at least at multiple occasions. Such data would have allowed us to examine if developmental trajectories of oppositionality are related to changes in children's adjustment across a 3-year period.

\section{Future research}

Some aspects of the assessment and development of oppositionality warrant further attention. One of these aspects is the distinction between oppositional emotions and oppositional behavior. The present thesis showed that these two types of oppositional 
responses can be distinguished, but are also strongly related to each other, suggesting that oppositional behavior is to a large extent driven by emotions. Frustration and anger (in response to requests and prohibitions), and a lack of shame or guilt (resulting from disciplinary actions) may all substantially increase the readiness to engage in oppositional behavior (Chapter 2). However, we feel that the distinction between oppositional emotions and behaviors could be potentially important for studying the role of behavioral and emotional control in the development of oppositionality (Greene \& Doyle, 1999; Stifter et al., 1999). Future research could therefore take this distinction as the point of departure in studying developmental changes in oppositionality.

Future research efforts could also help to shed more light on why some individuals become increasingly oppositional during early adolescence and others become less oppositional. Although only a few studies have focused on predictors of change, the literature does provide some clues. A recent longitudinal study by Galambos, Barker, and Almeida (2003) focusing on trajectories of change in externalizing and internalizing problems in early adolescence suggests that parents exert an important influence. It was shown that externalizing problems increased for those individuals whose parents failed to regulate their child's behavior through firm control and clear limit setting. Schaeffer, Petras, Ialongo, Poduska, and Kellam (2003) examined trajectories of boys' aggressive behavior across elementary school. They found that boys whose aggression increased over time had more concentration problems at the start of the study. Together these findings suggest that hyperactivity and ineffective parenting may be important risk factors that can probably cause an aggravation of the child's initial oppositional problems.

In addition, more research is needed to understand the psychological mechanisms that can account for the observed relation between the developmental trajectories of oppositionality and negative outcomes. The findings strongly suggest that oppositional interactions cannot be seen as isolated events of problem behavior (Ruchkin \& Schwab-Stone, 2003). Oppositional behaviors are likely to set in motion a chain of negative events by limiting children's potential to learn socially competent forms of behavior and by their disruptive effect on social relationships. The cumulative disadvantages and negative consequences of oppositionality could ultimately lead to significant impairment within multiple domains of the child's functioning. Future research is needed to explicate and substantiate these proposed mechanisms. 


\section{Nederlandse Samenvatting}

\section{Het vaststellen en de ontwikkeling van opstandigheid}

Opstandigheid kenmerkt zich door een zekere tegendraadsheid; het kind doet niet wat men van hem of haar verlangt en houdt zich niet aan algemeen aanvaarde sociale regels. Opstandigheid is een verschijnsel dat op verschillende manieren en in verschillende situaties de kop op kan steken. Ingaan tegen de geboden en wensen van ouders en leerkrachten, emotionele uitbarstingen en mild agressief gedrag tegenover leeftijdgenoten kunnen alle uitingen van opstandigheid zijn.

Empirisch onderzoek naar opstandigheid is schaars. Veel onderzoeken maken geen onderscheid tussen opstandigheid, agressie en antisociaal gedrag en vatten al deze gedragingen samen onder de noemer gedragsproblemen of externaliserend probleemgedrag (Greene \& Doyle, 1999). Vanuit het idee dat opstandigheid eerder een voorloper van ernstigere antisociale problemen is dan onderdeel van een meer veelomvattende problematiek richt dit proefschrift zich specifiek op opstandigheid. Het eerste deel richt zich op factoren die van invloed zijn op het vaststellen van opstandig en lastig gedrag. Allereerst onderzochten we de mogelijkheid om opstandigheid te meten met behulp van een nieuwe vragenlijst: de Amsterdamse Schaal voor Opstandigheid (ASO). Daarnaast onderzochten we het probleem van situationele specificiteit bij het vaststellen van probleemgedrag in het algemeen. Het tweede deel bestaat uit twee studies naar de ontwikkeling van opstandigheid in de vroege adolescentie (9-13 jaar). Deze studies bieden zowel inzicht in het ontwikkelingsbeloop van opstandigheid alsmede inzicht in de relatie tussen individuele verschillen in de ontwikkeling van opstandigheid en het latere functioneren van het kind op een aantal belangrijke gebieden. Omdat alle studies in dit proefschrift gebruik maken van de ASO lijkt het ons gepast eerst een goed beeld te schetsen van dit instrument alvorens dieper in te gaan op elk van de afzonderlijke studies.

De Amsterdamse Schaal voor Opstandigheid (ASO) is geconstrueerd om bij kinderen van acht tot twaalf jaar te kunnen vaststellen hoe opstandig ze zijn (Lagendijk, 1997; Leeuwen, 1996; Matten, 1995). De ASO is geconstrueerd met het uitgangspunt dat opstandigheid te beschrijven valt door een continuüm. Normaal verzet tegen de invloed van ouders en bemoeienissen van leerkrachten zijn aan het begin van dit continuüm te plaatsen. Woede-uitbarstingen, extreme ongehoorzaamheid en koppigheid vormen het andere uiteinde van dit continuüm. De ASO is een zelfrapportage vragenlijst met 30 gedwongen keuze items. Het kind heeft telkens de keuze tussen een opstandig alternatief en een niet-opstandig alternatief. Geprobeerd is om beide alternatieven even aantrekkelijk of juist onaantrekkelijk te 
maken door bijvoorbeeld als alternatief voor het opstandige antwoord een wel erg brave en daarom onaantrekkelijke formulering te kiezen. Een voorbeeld hiervan is het volgende item (score tussen haakjes): niet doen wat de juf/meester zegt, vind ik stoer (1) vind ik stom (0). De items van de ASO hebben alle betrekking op alledaagse, veelvoorkomende situaties zoals die zich voordoen in interacties met ouders, leerkrachten en andere autoriteitsfiguren, en leeftijdgenoten. Daarnaast is er aandacht voor zowel opstandige gevoelens als opstandige gedragingen in deze situaties. Het volgende voorbeelditem vraagt naar opstandig gedrag in situaties met ouders (score tussen haakjes): als mijn vader of moeder zegt dat ik moet helpen, doe ik dat (0) doe ik alsof ik dat niet hoor (1), terwijl het volgende item gevoelens op school beschrijft: als ik van de juf/meester straf krijg, dan voel ik mij verdrietig (0) dan word ik $k w a a d$ (1). Een voorbeeld van een item dat betrekking heeft op opstandige gevoelens in situaties met leeftijdgenoten is: als ik ruzie heb met andere kinderen, is dat maar kort (0) blijf ik lang kwaad (1).

\section{Het vaststellen van opstandig en lastig gedrag}

Hoofdstuk 2 biedt een meer gedetailleerde beschrijving van de theoretische uitgangspunten die gehanteerd zijn bij de constructie van de ASO en beschrijft een studie naar de structurele validiteit van dit meetinstrument. Het betreft hier een cross-sectioneel onderzoek gebaseerd op een representatieve landelijke steekproef van 1196 jongens en meisjes in de leeftijd van 8 tot 13 jaar (groep 6, 7, en 8 van de basisschool). Door verschillende factormodellen te toetsen werd de structuur van opstandigheid onderzocht. De interne structuur van de ASO werd volledig empirisch gereproduceerd. Het zogenaamde bestpassende structurele model bevatte een emotie en gedragsfactor, en drie situatiefactoren. Uit de resultaten blijkt dat opstandigheid het best gezien kan worden als een combinatie van opstandige gevoelens en opstandige gedragingen in verschillende situaties. Verder blijkt dat opstandigheid voor een deel afhankelijk is van de situatie. Sommige kinderen zorgen met name thuis voor overlast door hun opstandigheid, terwijl andere juist op school aanleiding tot problemen geven doordat zij zich met name verzetten tegen de leerkracht.

Om de bruikbaarheid van de ASO verder na te gaan werd in hoofdstuk 3 de criteriumgerelateerde validiteit onderzocht. Voor dit doel werden de scores van de ASO gerelateerd aan ouderoordelen en leerkrachtoordelen op de Strengths and Difficulties Questionnaire (SDQ; Goodman, 1997). De studie maakte gebruik van de gegevens van dezelfde steekproef als in hoofdstuk 2. De resultaten waren overeenkomstig de verwachtingen en lieten zien dat de zelfgerapporteerde mate van opstandigheid positief samenhangt met gedragsproblemen $(\mathrm{r}=.41)$ en hyperactiviteit $(\mathrm{r}=.38)$ zoals geobserveerd door ouders en leerkrachten. Daarnaast bleek opstandigheid gemeten met behulp van de ASO negatief samen te hangen met pro-sociaal gedrag zoals geobserveerd door ouders en leerkrachten $(r=-.36)$. De relatie tussen opstandigheid en de gemeten criteria bleek te variëren over situaties en informanten. De bevindingen ondersteunen de validiteit van de ASO. Ze suggereren tevens 
dat door kinderen zelf gerapporteerde gevoelens en gedragingen in verschillende situaties inzicht kunnen geven in de situationele invloeden op opstandigheid.

Zowel in onderzoek als in de klinische praktijk wordt vaker vertrouwd op de informatie van ouders en leerkrachten dan zelfrapportage door (jonge) kinderen. Het gebrek aan overeenstemming tussen deze verschillende informanten vormt echter een probleem (Achenbach, McConaughy, \& Howell, 1987). Ouders en leerkrachten verschillen vaak in hun oordeel over gedragsproblemen van kinderen. Het onderzoek beschreven in hoofdstuk 4 had tot doel beter te begrijpen waarom de oordelen van verschillende informanten verschillen. De volgende factoren worden verondersteld van invloed te zijn op de mate van overeenkomst tussen ouders en leerkrachten: a) situationele verschillen in het gedrag van het kind, b) verschillen tussen leerkrachten onderling in hun opvattingen en normen over lastig gedrag, en c) het gebrek aan zichtbaarheid van meer verborgen en stiekeme probleemgedragingen (bijv. stelen). Hoofdstuk 4 maakte gebruik van recent ontwikkelde structurele modellen voor gegevens van meerdere informanten (Bollen \& Paxton, 1998) en zelfgerapporteerde opstandigheid in verschillende situaties om de invloed van deze factoren te kunnen onderzoeken. De resultaten laten zien dat bovengenoemde factoren daadwerkelijk van invloed zijn op de mate van overeenkomst tussen ouders en leerkrachten in hun oordelen over gedragsproblemen. Leerkrachten bleken unieke situatiespecifieke informatie over de gedragsproblemen van kinderen te geven. Op grond van de resultaten lijkt het niet zinvol om voor het vaststellen van lastig en opstandig gedrag de voorkeur te geven aan informatie van ouders of juist aan informatie van de leerkracht. Beide informanten blijken verschillende, maar betekenisvolle informatie over het kind te geven.

\section{De ontwikkeling van opstandigheid}

De periode rond het tweede levensjaar staat wel bekend als "ik ben twee dus zeg ik nee" of de "terrible twos" omdat veel kinderen juist op die leeftijd vaak precies het tegenovergestelde lijken te doen van wat ouders graag zouden willen. Lastig en ongehoorzaam gedrag kunnen ook na de "terrible twos" een probleem vormen voor ouders. Bij velen heerst het idee dat met name aan het einde van de basisschool opstandigheid weer tot bloei kan komen. De laatste twee studies van dit proefschrift (hoofdstuk 5 en 6 ) hadden tot doel inzicht te krijgen in de ontwikkeling van opstandigheid gedurende de vroege adolescentie (9-13 jaar). De studies zijn gebaseerd op gegevens van 498 kinderen (242 jongens en 256 meisjes). Gedurende de laatste drie jaren van de basisschool zijn elk half jaar gegevens verzameld over deze kinderen. Van de meerderheid zijn gegevens beschikbaar over vijf of zes meetmomenten.

Hoofdstuk 5 had tot doel de ontwikkeling van opstandigheid gedurende de vroege adolescentie te beschrijven. De onder het grote publiek breed gedeelde opvatting dat opstandigheid toeneemt in deze periode wordt ondersteund door onderzoek dat laat zien dat een aanzienlijk deel van alle jongeren een periode van 'storm en stress' doormaakt (Arnett, 
1999). Longitudinaal onderzoek naar de ontwikkeling van opstandigheid spreekt het algemeen heersende beeld echter tegen. Opstandig gedrag blijkt gestaag af te nemen naarmate kinderen ouder worden (Bongers e.a., 2004; Lahey e.a., 2000; Nagin \& Tremblay, 1999).

Het onderzoek beschreven in hoofdstuk 5 richt zich specifiek op de ontwikkeling van opstandigheid gedurende de vroege adolescentie. Daarnaast werd in tegenstelling tot eerder longitudinaal onderzoek aandacht gegeven aan situationele verschillen in de ontwikkeling van opstandigheid. De resultaten verschillen van die van eerdere studies en laten zien dat kinderen over het algemeen opstandiger worden gedurende de laatste drie jaren van de basisschool. Uit het onderzoek bleek tevens dat er grote individuele verschillen in de ontwikkeling van opstandigheid zijn. Sommige kinderen worden steeds opstandiger, anderen worden juist minder opstandig en voor weer anderen geldt dat er weinig verandert in hun gedrag en gevoelens. Deze bevindingen tezamen ondersteunen de gedachte dat storm en stress kenmerkend zijn voor vele jonge adolescenten, maar zeker niet voor allen (Arnett, 1999). Verder bleek dat zowel het algemene ontwikkelingsbeloop alsmede individuele verschillen in de ontwikkeling van opstandigheid deels situatie specifiek waren. Deze bevindingen wijzen erop dat de ontwikkeling van opstandigheid deels bepaald wordt door de situatie. De ontwikkeling van gedragsproblemen wordt vaak opgevat als een proces dat zich binnen het individu voltrekt. De resultaten van dit onderzoek suggereren dat de ontwikkeling van opstandigheid een proces is dat open staat voor situationele invloeden en geven derhalve aanleiding tot een kijk op de ontwikkeling van gedragsproblemen waarin meer ruimte is voor de sociale context.

Het onderzoek beschreven in hoofdstuk 6 had tot doel de betekenis van opstandigheid in de vroege adolescentie na te gaan. Een toename in opstandigheid gedurende deze periode kan duiden op een problematische ontwikkeling die uiteindelijk leidt tot antisociaal gedrag, verslechterde schoolprestaties en problemen in sociale relaties, maar een zelfde ontwikkelingstraject zou ook een afspiegeling kunnen zijn van een meer kritische houding ten opzichte van autoriteitsfiguren en het streven naar meer controle en autonomie. Omdat de ontwikkeling van opstandigheid in de vroege adolescentie zowel nadelige uitkomsten (b.v. verminderde sociale steun) als gunstige uitkomsten (b.v. meer autonomie en een hogere zelfwaardering) zou kunnen voorspellen zijn twee concurrerende hypothesen geformuleerd. De 'risico hypothese' gaat uit van de nadelige gevolgen van opstandigheid. Volgens deze hypothese leidt opstandigheid tot problemen op meerdere gebieden. De 'competentie verhogende hypothese' gaat uit van de veronderstelling dat opstandigheid een vereiste is om een zekere mate van autonomie te verwerven. Volgens deze hypothese leidt opstandigheid tot een hogere mate van autonomie en een positiever zelfbeeld.

In het onderzoek werd gebruik gemaakt van de longitudinale gegevens waarop ook hoofdstuk 5 gebaseerd is. Om de twee concurrerende hypothesen te toetsen werd de relatie tussen individuele ontwikkelingstrajecten van opstandigheid en een breed scala aan uitkomsten onderzocht. De uitkomsten werden gemeten aan het einde van de basisschool; een 
tijdstip waarop kinderen geacht worden rijp genoeg te zijn voor de overgang naar een meer volwassen schoolsysteem. De resultaten laten zien dat ontwikkelingstrajecten van opstandigheid alleen negatieve uitkomsten voorspellen. Zowel een hoog gemiddeld niveau van opstandigheid als een relatief sterke toename in opstandigheid gedurende de vroege adolescentie bleken een verhoogd risico te vormen voor slechtere schoolprestaties, een gebrek aan sociale steun, zwakke sociale vaardigheden, antisociaal gedrag, en psychopathologie. De resultaten zijn in overeenstemming met de 'risico hypothese'. Verder blijkt duidelijk dat leeftijdsgebonden veranderingen in zelfgerapporteerde opstandigheid van voorspellende waarde kunnen zijn voor het latere functioneren van het kind. Deze bevindingen ondersteunen de validiteit van zelfrapportage. Ze wijzen tevens op de mogelijkheid om kinderen met een verhoogd risico wellicht beter te kunnen identificeren door middel van herhaalde metingen.

De bovengenoemde bevindingen moeten gezien worden in het licht van de bestudeerde ontwikkelingsperiode. Zo kenmerken de ontwikkelingstrajecten van meisjes en jongens die rond hun $13^{\mathrm{e}}$ levensjaar aangeven wel eens gerookt te hebben zich door een relatief hoog niveau van opstandigheid en een sterke toename in opstandigheid. $\mathrm{Nu}$ ligt roken voor 13 a 14 jarigen misschien nog in het verlengde van opstandig gedrag, maar twee jaar later $(16,17$ jaar) zou het zo kunnen zijn dat niet roken eerder een uiting van opstandigheid is. Toekomstig onderzoek zou gebruik kunnen maken van een follow-up meting om te onderzoeken of de nadelige gevolgen van opstandigheid ook nog zichtbaar zijn in de (jong) volwassenheid.

In de algemene discussie in hoofdstuk 7 worden de belangrijkste empirische bevindingen besproken aan de hand van een aantal centrale thema's. Daarnaast werd ingegaan op de implicaties van de resultaten en mogelijkheden voor vervolgonderzoek. Geconcludeerd werd dat kinderen zelf betrouwbare en valide informatie kunnen geven over hun opstandige gevoelens en gedragingen in meerdere situaties door het invullen van de Amsterdamse Schaal voor Opstandigheid (ASO). Kinderen kunnen dus mogelijk een waardevolle bijdrage leveren aan (behandelingsgerichte) diagnostiek. De bevindingen laten tevens zien dat de diagnostiek van opstandigheid baat kan hebben bij meer aandacht voor situationele specificiteit, (snelle) veranderingen in de vroege adolescentie en geslachtsverschillen. Voor toekomstig onderzoek lijkt het belangrijk verklaringen te vinden voor interindividuele verschillen in intraindividuele veranderingen in opstandigheid. Met andere woorden: waarom worden sommige kinderen opstandiger en andere juist braver? Daarnaast is onderzoek nodig naar de specifieke risico mechanismen die ten grondslag liggen aan de relatie tussen de ontwikkeling van opstandigheid en negatieve uitkomsten op latere leeftijd. 



\section{References}

Abrahamson, A. C., Baker, L. A., \& Caspi, A. (2002). Rebellious teens? Genetic and environmental influences of the social attitudes of adolescents. Journal of Personality and Social Psychology, 83, 1392-1408.

Achenbach, T. M. (1978). Psychopathology of childhood: Research problems and issues. Journal of Consulting \& Clinical Psychology, 46, 759-776.

Achenbach, T. M. (1993). Empirically based taxonomy: How to use syndromes and profile types derived from the $C B C L / 4-18, T R F, \& Y S R$. Burlington, VT: UVM Department of Psychiatry.

Achenbach, T. M., McConaughy, S. H., \& Howell, C. T. (1987). Child/adolescent behavioral and emotional problems: Implications of cross-informant correlations for situational specificity. Psychological Bulletin, 101, 213-232.

Alexander, J. F., \& Pugh, C. A. (1996). Oppositional behavior and conduct disorders of children and youth. In F. W. Kaslow (Ed.), Handbook of relational diagnosis and dysfunctional family patterns (pp. 210-224). New York: John Wiley \& Sons.

American Psychiatric Association. (1994). Diagnostic and Statistical Manual of Mental Disorders, 4th edition (DSM-IV). Washington, DC: American Psychiatric Association.

Angold, A., \& Costello, E. J. (1996). The relative diagnostic utility of child and parent reports of oppositional defiant behaviors. International Journal of Methods in Psychiatric Research, 6, 253-259.

Anthony, E. J. (1976). The genesis of oppositional behavior. In A. J. Anthony \& D. C. Gilpin (Eds.), Three clinical faces of childhood (pp. 1-8). New York: Spectrum.

Arnett, J. J. (1999). Adolescent storm and stress, reconsidered. American Psychologist, 54, 317-326.

Baillargon, R. H., Boulerice, B., Tremblay, R. E., Zoccolillo, M., Vitaro, F., \& Kohen, D. E. (2001). Modeling interinformant agreement in the absence of a "gold standard". Journal of Child Psychology and Psychiatry, 42, 463-473.

Baltes, P. B., \& Nesselroade, J. R. (1979). History and rationale of longitudinal research. In J. R. Nesselroade \& P. B. Baltes (Eds.), Longitudinal research in the study of behavior and development (pp. 1-59). New York: Academic Press.

Barkley, R. A. (2003). Issues in the diagnosis of attention-deficit/hyperactivity disorder in children. Brain and Development, 25, 77-83.

Bauer, D. J., \& Curran, P. J. (2003a). Distributional assumptions of growth mixture models: Implications for overextraction of latent trajectory classes. Psychological Methods, 8 , 338-363.

Bauer, D. J., \& Curran, P. J. (2003b). Overextraction of latent trajectory classes: Much ado about nothing? Reply to Rindskopf (2003), Muthen (2003), and Cudeck and Henly (2003). Psychological Methods, 8, 384-393.

Baumrind, D. (1997). The discipline encounter: Contemporary issues. Aggression and violent behavior, 2, 321-355. 
Bennett, K. J., Lipman, E. L., Racine, Y., \& Offord, D. R. (1998). Annotation: Do measures of externalising behaviour in normal populations predict later outcome?: Implications for targeted interventions to prevent conduct disorder. Journal of Child Psychology and Psychiatry, 39, 1059-1070.

Bentler, P. M. (1990). Comparative fix indexes in structural models. Psychological Bulletin, 107, 238-246.

Bentler, P. M. (1995). EQS Structural Equations Program Manual. Encino, CA: Multivariate Software.

Bergman, L., Magnusson, D., \& El Khouri, B. M. (2003). Studying individual development in an interindividual context. (Vol. 4). Mahwah: Lawrence Erlbaum Associates.

Bernaards, C. A., \& Sijtsma, K. (2000). Influence of imputation and EM methods when item nonresponse is nonignorable. Multivariate Behavioral Research, 35, 321-364.

Biesanz, J. C., Deeb-Sossa, N., Papadakis, A. A., Bollen, K. A., \& Curran, P. J. (2004). The role of coding time in estimating and interpreting growth curve models. Psychological Methods, 9, 30-52.

Bigelow, B. J., Tesson, G., \& Lewko, J. H. (1996). Learning the rules: the anatomy of children's relationships. New York: The Guilford Press.

Blashfield, R. K., \& Livesley, W. J. (1991). Metaphorical analysis of psychiatric classification as a psychological test. Journal of Abnormal Psychology, 100, 262-270.

Bollen, K. A. (1989). Structural equations with latent variables. New York: Wiley.

Bollen, K. A., \& Paxton, P. (1998). Detection and determinants of bias in subjective measures. American Sociological Review, 63, 465-478.

Bongers, I. L., Koot, H. M., van der Ende, J., \& Verhulst, F. C. (2004). Developmental trajectories of externalizing behaviors in childhood and adolescence. Child Development, 75, 1523-1537.

Bownas, D. A., \& Bernardin, H. J. (1991). Surpressing illusory halo with forced-choice items. Journal of Applied Psychology, 76, 592-594.

Boyce, W. T., Frank, E., Jensen, P. S., Kessler, R. C., Nelson, C. A., Steinberg, L., \& The Macarthur foundation research network on psychopathology and development. (1998). Social context in developmental psychopathology: Recommendatoins for future research from the MacArthur network on psychopathology and development. Development and Psychopathology, 10, 143-164.

Boyle, M. H., \& Willms, J. D. (2001). Multilevel modelling of hierarchical data in developmental studies. Journal of Child Psychology and Psychiatry, 42, 141-162.

Braine, L. G., Pomerantz, E., Lorber, D., \& Krantz, D. H. (1992). Conflicts with authority: Children's feelings, actions, and justifications. Developmental Psychology, 27, 829840.

Braungart-Rieker, J., Garwood, M. M., \& Stifter, C. A. (1997). Compliance and noncompliance: The roles of maternal control and child temperament. Journal of Applied Developmental Psychology, 18, 411-428. 
Brehm, S. (1981). Oppositional behavior in children. In S. Brehm, S. M. Kassin, \& F. K. Gibbens (Eds.), Developmental social psychology: Theory and research (pp. 96-121). New York: Oxford University Press.

Brody, G. H., Murry, V. M., Kim, S., \& Brown, A. C. (2002). Longitudinal pathways to competence and psychological adjustment among African American children living in rural single-parent households. Child Development, 73, 1505-1516.

Broidy, L. M., Nagin, D. S., Tremblay, R. E., Bates, J. E., Brame, B., Dodge, K. A., Fergusson, D., Horwood, J. L., Loeber, R., Laird, R., Lynam, D. R., Moffitt, T. E., Pettit, G. S., \& Vitaro, F. (2003). Developmental trajectories of childhood disruptive behaviors and adolescent delinquency: A six-site, cross-national study. Developmental Psychology, 39, 222-245.

Bryk, A. S., \& Raudenbush, S. W. (1987). Application of hierarchical linear models to assessing change. Psychological Bulletin, 101, 147-158.

Bugental, D. B. (2000). Acquisition of the algorithms of social life: A domain-based approach. Psychological Bulletin, 126, 187-219.

Burns, G. L., Walsh, J. A., \& Gomez, R. (2003). Convergent and discrimant validity of trait and source effects in ADHD-inattention and hyperactivity/impulsivity measures across a 3-month interval. Journal of Abnormal Child Psychology, 31, 529-541.

Burns, G. L., Walsh, J. A., Patterson, D. R., Holte, C. S., Sommers-Flanagan, R., \& Parker, C. M. (1997). Internal validity of the disruptive behavior disorder symptoms: Implications from parent ratings for a dimensional approach to symptom validity. Journal of Abnormal Child Psychology, 25, 307-319.

Catalano, R. F., Mazza, J. J., Harachi, T. W., Abbott, R. D., Haggerty, K. P., \& Fleming, C. B. (2003). Raising healthy children through enhancing social development in elementary school: Results after 1.5 years. Journal of School Psychology, 41, 143-164.

CBS (Dutch Central Bureau for Statistics). (1997). Kwartaalschrift Onderwijsstatistieken. (Vol. 4(1)).

Chan, D., Ramey, S., Ramey, C., \& Schmitt, N. (2000). Modeling intraindividual changes in children's social skills at home and at school: A multivariate latent growth approach to understanding between-settings differences in children's social skills development. Multivariate Behavioral Research, 35, 365-396.

Cohen, J. (1988). Statistical power analysis for the behavioral sciences. Hillsdale, NJ: Erlbaum.

Cole, D. A., Tram, J. M., Martin, J. M., Hoffman, K. B., Ruiz, M. D., Jacquez, F. M., \& Maschman, T. L. (2002). Individual differences in the emergence of depressive symptoms in children and adolescents: A longitudinal investigation of parent and child reports. Journal of Abnormal Psychology, 111, 156-165.

Cole, P. M., Michel, M. K., \& Teti, L. O. D. (1995). The development of emotion regulation and dysregulation: A clinical perspective. Monographs of the Society for Research in Child Development, 59, 250-283. 
Cole, P. M., \& Zahn-Waxler, C. (1992). Emotional dysregulation in disruptive behavior disorders. In D. Cicchetti \& S. L. Toth (Eds.), Developmental Perspectives on Depression (4 ed., pp. 173-210). New York: University of Rochester Press.

Collins, L. M., \& Sayer, A. G. (2001). New methods for the analysis of change. Washington, DC: American Psychological Association.

Collins, W. A. (1997). Relationships and development during adolescence: Interpersonal adaptation to individual change. Personal Relationships, 4, 1-14.

Côté, S., Zoccolillo, M., Tremblay, R. E., Nagin, D., \& Vitaro, F. (2001). Predicting girls' conduct disorder in adolescence from childhood trajectories of disruptive behaviors. Journal of the American Academy of Child and Adolescent Psychiatry, 40, 678-684.

Crick, N. R., \& Zahn-Waxler, C. (2003). The development of psychopathology in females and males: Current progress and future challenges. Development and Psychopathology, 15, 719-742.

Crockenberg, S., \& Litman, C. (1990). Autonomy as competence in 2-year olds: Maternal correlates of child defiance, compliance, and self-assertion. Developmental Psychology, 26, 961-971.

Culp, R. E., Howell, C. S., McDonald Culp, A., \& Blankemeyer, M. (2001). Maltreated children's emotional and behavioral problems: Do teachers and parents see the same things? Journal of Child and Family Studies, 10, 39-50.

Curran, P. J., \& Willougby, M. T. (2003). Implications of latent trajectory models for the study of developmental psychopathology. Development and Psychopathology, 15, 581-612.

Damon, W. (1988). The moral child: Nurturing children's moral growth. New York: The Free Press.

Demaray, M. K., Ruffalo, S. L., Carlson, J., Busse, R. T., Olson, A. E., McManus, S. M., \& Leventhal, A. (1995). Social skills assessment: A comparative evaluation of six published rating scales. School Psychology Review, 24, 648-672.

Denton, W. H. (1996). Problems encountered in reconciling individual and relational diagnoses. In F. W. Kaslow (Ed.), Handbook of relational diagnosis and dysfunctional family patterns (pp. 35-45). New York: John Wiley \& Sons.

Dodge, K. A. (1991). Emotion and social information processing. In J. Garber \& K. A. Dodge (Eds.), The development of emotion regulation and dysregulation (pp. 159181). Cambridge: Cambridge University Press.

Drabick, D., A.G., Strassberg, Z., \& Kees, M. R. (2001). Measuring qualitative aspects of preschool boys' noncompliance: The response style questionnaire (RSQ). Journal of Abnormal Child Psychology, 29, 129-139.

Driessen, G. (2002). School composition and achievement in primary education: A large-scale multilevel approach. Studies in Educational Evaluation, 28, 347-368.

DuPaul, G. J. (2003). Assessment of ADHD symptoms: Comment on Gomez et al. (2003). Psychological Assessment, 15, 115-117. 
Eddowes, E. A., Aldridge, J., \& Culpepper, S. (1994). Primary teachers' classroom practices and their perceptions of children's attention problems. Perceptual and Motor Skills, 79, 787-790.

Edmundson, E. W., Koch, W. R., \& Silverman, S. (1993). A facet analysis approach to content and construct validity. Educational \& Psychological Measurement, 53, 351369.

Eid, M., Lischetzke, T., Nussbeck, F. W., \& Trierweiler, L. I. (2003). Separating trait effects from trait-specific method effects in multitrait-multimethod models: A multipleindicator CT-C(M-1) model. Psychological Methods, 8, 38-60.

Eisenberg, N., Fabes, R. A., Guthrie, I. K., Murphy, B. C., Maszk, P., Holmgren, R., \& Suh, K. (1996). The relations of regulation and emotionality to problem behavior in elementary school children. Development \& Psychopathology, 8, 141-162.

Fergusson, D. M., \& Horwood, L. J. (1987). The trait and method components of ratings of conduct disorder. Part I.: Maternal and teacher evaluations of conduct disorder in young children. Journal of Child Psychology and Psychiatry, 28, 249-260.

Fergusson, D. M., \& Horwood, L. J. (1989). Estimation of method and trait variance in ratings of conduct disorder. Journal of Child Psychology and Psychiatry, 30, 365-378.

Fisher, P. A., \& Fagot, B. I. (1996). Development of consensus about child oppositional behavior: Increased convergence with entry into school. Journal of Applied Developmental Psychology, 17, 519-534.

Fiske, D. W. (1971). Measuring the concepts of personality. Chicago, Ill.: Aldine.

Fitzmaurice, G. M., Laird, N. M., Zahner, G. E. P., \& Daskalakis, C. (1995). Bivariate logistic regression analysis of childhood psychopathology ratings using multiple informants. American Journal of Epidemiology, 1194-1203.

Flory, K., Lynam, D., Milich, R., Leukefeld, C., \& Clayton, R. (2004). Early adolescent through young adult alcohol and marijuana use trajectories: Early predictors, young adult outcomes, and predictive utility. Development and Psychopathology, 16, 193 213.

Frauenglass, S., \& Routh, D. K. (1999). Assessment of the Disruptive Behavior Disorders. In H. C. Quay \& A. E. Hogan (Eds.), Handbook of the disruptive behavior disorders (pp. 49-74). New York: Kluwer Academic/Plenum Publishers.

Frick, P. J., Cornell, A. H., Bodin, S. D., Dane, H. E., Barry, C. T., \& Loney, B. R. (2003). Callous-unemotional traits and developmental pathways to severe conduct problems. Developmental Psychology, 39, 246-260.

Frick, P. J., Lahey, B. B., Loeber, R., Tannenbaum, L., Van Horn, Y., Christ, M. A. G., Hart, E. A., \& Hanson, K. (1993). Oppositional Defiant Disorder and Conduct Disorder: A meta-analytic review of factor analyses and cross-validation in a clinic sample.

Clinical Psychology Review, 13, 319-340.

Frijda, N. (1986). The emotions. Cambridge, UK: Cambridge University Press.

Friman, P. C., Handwerk, M. L., Smith, G. L., Larzelere, R. E., Lucas, C. P., \& Schaffer, D. M. (2000). External validity of conduct and oppositional defiant disorders determined 
by the NIMH diagnostic interview schedule for children. Journal of Abnormal Child Psychology, 28, 277-286.

Fuchs, D., \& Thelen, M. H. (1988). Children's expected interpersonal consequences of communicating their affective states and reported likelihood of expression. Child Development, 59, 1314-1322.

Furman, W., \& Buhrmester, D. (1985). Children's perceptions of the personal relationships in their social networks. Developmental Psychology, 21, 1016-1024.

Galambos, N. L., Barker, E. T., \& Almeida, D. M. (2003). Parents do matter: Trajectories of change in externalizing and internalizing problems in early adolescence. Child Development, 74, 578-594.

Gard, G. C., \& Berry, K. K. (1987). Oppositional children: Taming tyrants. Journal of Clinical Child Psychology, 15, 148-158.

Gerrits, M. H., van den Oord, E. J. C. G., \& Voogt, R. (2001). An evaluation of nonresponse bias in peer, self and teacher ratings of children's psychosocial adjustment. Journal of Child Psychology and Psychiatry, 42, 593-602.

Goldstein, H. (1989). Efficient prediction models for adult height. In J. M. Tanner (Ed.), Auxology 88: Advances in the science of growth and development (pp. 41-48). London: Smith-Gordon/Nishimura.

Goldstein, H. (1995). Multilevel statistical models (Second edition). London: Arnold.

Goldstein, H. (2003). Multilevel statistical models (Third edition). (Vol. 3). London: Edward Arnold.

Goldwasser, M. A., \& Fitzmaurice, G. M. (2001). Multivariate linear regression analyis of childhood psychopathology using multiple informant data. International Journal of Methods in Psychiatric Research, 10, 1-10.

Gomez, R., Burns, G. L., Walsh, J. A., \& Moura, M. A. d. (2003a). A multitrait-multisource confirmatory factor analytic approach to the construct validity of ADHD rating scales. Psychological Assessment, 15.

Gomez, R., Burns, G. L., Walsh, J. A., \& Moura, M. A. d. (2003b). Understanding source effect in ADHD rating scales: Reply to DuPaul (2003). Psychological Assessment, 15, 118-119.

Goodman, R. (1997). The Strengths and Difficulties Questionnaire: A research note. Journal of Child Psychology \& Psychiatry, 38, 581-586.

Goodman, R. (2001). Psychometric properties of the Strengths and Difficulties Questionnaire. Journal of the American Academy of Child and Adolescent Psychiatry, 40, 1337-1345.

Goodman, R., Ford, T., Simmons, H., Gatward, R., \& Meltzer, H. (2000). Using the Strengths and Difficulties Questionnaire (SDQ) to screen for child psychiatric disorders in a community sample. British Journal of Psychiatry, 534-539.

Graber, J. A., \& Brooks-Gunn, J. (1996). Transitions and turning points: Navigating the passage from childhood through adolescence. Developmental psychology, 32, 768776. 
Granic, I., Hollenstein, T., Dishion, T. J., \& Patterson, G. R. (2003). Longitudinal analysis of flexibility and reorganization in early adolescence: A dynamic systems study of family interactions. Developmental Psychology, 39, 606-617.

Greenbaum, P. E., Decrick, R. F., Prange, M. E., \& Friedman, R. M. (1994). Parent, teacher, and child ratings of problem behaviors of youngsters with serious emotional disturbances. Psychological Assessment, 6, 141-148.

Greene, R. W., Biederman, J., Zerwas, S., Monuteaux, M. C., Goring, J. C., \& Faraone, S. V. (2002). Psychiatric comorbidity, family dysfunction, and social impairment in referred youth with oppositional defiant disorder. American Journal of Psychiatry, 1214-1224.

Greene, R. W., \& Doyle, A. E. (1999). Toward a transactional model of oppositional defiant disorder: Implications for assessment and treatment. Clinical Child and Family Psychology Review, 2, 129-148.

Gresham, F. M., \& Elliott, S. N. (1990). The social skills rating system. Circle Pines, MN: American Guidance Service.

Grills, A. E., \& Ollendick, T. H. (2002). Issues in parent-child agreement: The case of structured diagnostic interviews. Clinical Child and Family Psychology Review, 5, 5783.

Hart, E. L., Lahey, B. B., Loeber, R., \& Hanson, K. (1994). Criterion validity of informants in the diagnosis of disruptive behavior disorders in children: A preliminary study. Journal of Consulting and Clinical Psychology, 62, 410-414.

Harter, S. (1985). Manual for the social support scale for children. Denver, CO: University of Denver.

Harter, S. (1988). Manual for the self-perception profile for children. Denver, CO: University of Denver.

Haselager, G. J. T., Cillesen, A. H. N., Van Lieshout, C. F. M., Riksen-Walraven, J. M. A., \& Hartup, W. W. (2002). Heterogeneity among peer-rejected boys across middle childhood: Developmental pathways of social behavior. Developmental Psychology, $38,446-456$.

Hastings, P. D., Zahn-Waxler, C., Robinson, J., Usher, B., \& Bridges, D. (2000). The development of concern for others in children with behavior problems. Developmental Psychology, 36, 531-546.

Hemphill, J. F. (2003). Interpreting the magnitudes of correlation coefficients. American Psychologist, 58, 78-79.

Hendren, R. L. (1999). Foreword. In R. L. Hendren (Ed.), Disruptive behavior disorders in children and adolescents (Vol. 18, pp. xiii-xvii). Washington DC: American Psychiatric Press.

Henker, B., \& Whalen, C. K. (1999). The child with Attention-Deficit/Hyperactivity Disorder in school and peer settings. In H. C. Quay \& A. E. Hogan (Eds.), Handbook of disruptive behavior disorders (pp. 157-178). New York: Kluwer Academic/Plenum.

Henning-Stout. (1998). Assessing the behavior of girls: What we see and what we miss. Journal of School Psychology, 36, 433-455. 
Hertzog, C., \& Nesselroade, J. R. (2003). Assessing psychological change in adulthood: An overview of methodological issues. Psychology and Aging, 18, 639-657.

Hill, L. G., Lochman, J. E., Coie, J. D., Greenberg, M. T., and Conduct Problems Prevention Research Group (in press). Effectiveness of early screening for externalizing problems: Issues of screening accuracy and utility. Journal of Consulting and Clinical Psychology.

Hinshaw, S. P. (2002). Process, mechanism, and explanation related to externalizing behavior in developmental psychopathology. Journal of Abnormal Child Psychology, 30, 431446.

Hinshaw, S. P., \& Anderson, C. A. (1997). Conduct and oppositional defiant disorders. In E. J. Mash \& R. A. Barkley (Eds.), Child psychopathology (pp. 113-149). New York: Guilford Press.

Hinshaw, S. P., \& Park, T. (1999). Research problems and issues: Toward a more definitve science of disruptive disorders. In H. C. Quay \& A. E. Hogan (Eds.), Handbook of disruptive behavior disorders (pp. 593-620). New York: Kluwer Academic/Plenum Publishers.

Hoeksma, J. B., \& Knol, D. L. (2001). Testing predictive developmental hypotheses. Multivariate Behavioral Research, 36, 227-248.

Hoeksma, J. B., \& Koomen, H. M. Y. (1992). Multilevel models in developmental psychological research: Rationales and applications. Early Development and Parenting, 1, 157-167.

Hoffenaar, P. J., \& Hoeksma, J. B. (2002). The structure of oppositionality: Response dispositions and situational aspects. Journal of Child Psychology and Psychiatry, 43, 375-385.

Hoffenaar, P. J., Hoeksma, J. B., \& Koot, H. M. (submitted). Self-reported oppositionality: Individual and situational differences. Journal of Clinical Child and Adolescent Psychology.

Hope, T. L., \& Bierman, K. L. (1998). Patterns of home and school behavior problems in rural and urban settings. Journal of School Psychology, 36, 45-58.

Hsieh, D. K., \& Kirk, S. A. (2003). The effect of social context on psychiatrists' judgments of adolescent antisocial behavior. Journal of Child Psychology and Psychiatry, 44, 877887.

Huisman, M. (1999). Item Nonresponse: Occurence, Caues, and Imputation of Missing Answers to Test Items. Leiden: DSWO Press.

Jackson, D. N., Wroblewski, V. R., \& Ashton, M. C. (2000). The impact of faking on employment tests: Does forced choice offer a solution? Human Performance, 13, 371388.

Jensen, P. S., Brooks-Gunn, J., \& Graber, J. A. (1999). Dimensional scales and diagnostic categories: Constructing crosswalks for child psychopathology assessments Introduction. Journal of the American Academy of Child \& Adolescent Psychiatry, 38, 118-120. 
Johnston, C., \& Murray, C. (2003). Incremental validity in the psychological assessment of children and adolescents. Psychological Assessment, 15, 496-507.

Joormann, J., \& Stöber, J. (1997). Measuring facets of worry: A lisrel analysis of the worry domains questionnaire. Personality and individual differences, 23, 827-837.

Jöreskog, K. G., \& Sörbom, D. (1996). LISREL 8 User's Reference Guide. Chicago: Scientific Software International.

Kalb, L. M., \& Loeber, R. (2003). Child disobedience and noncompliance: A review. Pediatrics, 111, 641-652.

Kamphaus, R. W., DiStefano, C., \& Lease, A. M. (2003). A self-report typology of behavioral adjustment for young children. Psychological Assessment, 15, 17-28.

Kavanagh, K., \& Hops, H. (1994). Good girls? Bad boys? Gender and development as contexts for diagnosis and treatment. Advances in Clinical Child Psychology, 16, 4579.

Kazdin, A. E., Kraemer, H. C., Kessler, R. C., Kupfer, D. J., \& Offord, D. R. (1997). Contributions of risk-factor research to developmental psychopathology. Clinical Psychology Review, 17, 375-406.

Keiley, M. K., Bates, J. E., Dodge, K. A., \& Pettit, G. S. (2000). A cross-domain growth analysis: Externalizing and internalizing behaviors during 8 years of childhood. Journal of Abnormal Child Psychology, 28, 161-179.

Kim, S., \& Brody, G. H. (2003). Linking self-regulation change to parent-child relationships, behavioral competence, and psychosocial maturity during early adolescence. Paper presented at the Annual meeting of the American Educational Research Association (AERA), Chicago, IL.

Kokko, K., \& Pulkkinen, L. (2000). Aggression in childhood and long-term unemployment in adulthood: A cycle of maladaptation and some protective factors. Developmental Psychology, 36, 463-472.

Koops, W., \& Zuckerman, M. (2003). Introduction: A historical developmental approach to adolescence. History of the Family, 8, 345-354.

Kuczynski, L., \& Hildebrandt, N. (1997). Models of conformity and resistance in socialization theory. In J. E. Grusec \& L. Kuczynski (Eds.), Parenting and children's internalization of values: A handbook of contemporary theory (pp. 227-256). New York: Wiley.

Kuczynski, L., \& Kochanska, G. (1990). Development of children's noncompliance strategies from toddlerhood to age 5. Developmental Psychology, 26, 398-408.

Kuo, M., Mohler, B., Raudenbush, S. L., \& Earls, F. J. (2000). Assessing exposure to violence using multiple informants: Application of hierarchical linear model. Journal of Child Psychology and Psychiatry, 41, 1049-1056.

Lagendijk, E. H. (1997). Tweede revisie van de Amsterdamse Schaal voor Opstandig gedrag. Unpublished Manuscript, Free University, Amsterdam.

Lahey, B. B., Loeber, R., Burke, J., Rathouz, P. J., \& McBurnett, K. (2002). Waxing and waning in concert: Dynamic comorbidity of conduct disorder with other disruptive and 
emotional problems over 7 years among clinic-referred boys. Journal of Abnormal Psychology, 111, 556-567.

Lahey, B. B., Schwab-Stone, M., Goodman, S. H., Waldman, I. D., Canino, G., Rathouz, P. J., Miller, T. L., Dennis, K. D., Hector, B., \& Jensen, P. S. (2000). Age and gender differences in oppositional behavior and conduct problems: A cross-sectional household study of middle childhood and adolescence. Journal of Abnormal Psychology, 109, 488-503.

Lance, C. E., Noble, C. L., \& Scullen, S. E. (2002). A critique of the correlated traitcorrelated method and correlated uniqueness models for multitrait-multimethod data. Psychological Methods, 7, 228-244.

Langbehn, D. R., Cadoret, R. J., Yates, W. R., Troughton, E. P., \& Stewart, M. A. (1998). Distinct contributions of conduct and oppositional defiant symptoms to adult antisocial behavior: Evidence from an adoption study. Archives of General Psychiatry, 55, 821829.

Larson, R., \& Ham, M. (1993). Stress and "storm and stress" in early adolescence: The relationship of negative events with dysphoric affect. Developmental Psychology, 29, 130-140.

Larson, R. W., Moneta, G., Richards, M. H., \& Wilson, S. (2002). Continuity, stability, and change in daily emotional experience across adolescence. Child Development, 73, 1151-1165.

Laupa, M., Turiel, E., \& Cowan, P. A. (1995). Obedience to authority in children and adults. In M. Killen \& D. Hart (Eds.), Morality in everyday life: Developmental perspectives. (pp. 131-165). New York: Cambridge University Press.

Laursen, B., Pulkkinen, L., \& Adams, R. (2002). The antecedents and correlates of agreeableness in adulthood. Developmental Psychology, 38, 591-603.

Leadbeater, B. J., Kuperminc, G. P., Blatter, S. J., \& Hertzog, C. (1999). A multivariate model of gender differences in adolescents' internalizing and externalizing problems. Developmental Psychology, 35, 1268-1282.

Ledingham, J. E. (1999). Children and adolescents with Oppositional Defiant Disorder and Conduct Disorder in the community. In H. C. Quay \& A. E. Hogan (Eds.), Handbook of disruptive behavior disorders (pp. 353-370). New York: Kluwer Academic/Plenum.

Leeuwen, J. v. (1996). Eerste revisie van de Amsterdamse Schaal voor Opstandig gedrag. Unpublished Manuscript, Free University, Amsterdam.

Leman, P. J., \& Duveen, G. (1999). Representations of authority and children's moral reasoning. European Journal of Social Psychology, 29, 557-575.

Loeber, R., Burke, J. D., Lahey, B. B., Winters, A., \& Zera, M. (2000). Oppositional Defiant and Conduct Disorder: A review of the past 10 years, part I. Journal of the American Academy of Child and Adolescent Psychiatry, 39, 1468-1484.

Loeber, R., Green, S., Lahey, B. B., \& Stouthamer-Loeber, M. (1989). Optimal informants on childhood disruptive behavior disorders. Development and Psychopathology, 1, 317337. 
Loeber, R., Green, S. M., Keenan, K., \& Lahey, B. B. (1995). Which boys will fare worse? Early predictors of the onset of conduct disorder in a six-year longitudinal study. Journal of the American Academy of Child \& Adolescent Psychiatry, 34, 499-509.

Loeber, R., Green, S. M., \& Lahey, B. B. (1990). Mental health professionals' perception of the utility of children, mothers and teachers as informants on childhood psychopathology: A review. Journal of Clinical Child Psychology, 19, 136-143.

Loeber, R., Green, S. M., Lahey, B. B., \& Stouthamer-Loeber, M. (1991a). Differences and similarities between children, mothers, and teachers as informants on disruptive child behavior. Journal of Abnormal Child Psychology, 19, 75-95.

Loeber, R., Lahey, B. B., \& Thomas, C. (1991b). Diagnostic conundrum of oppositional defiant disorder and conduct disorder. Journal of Abnormal Psychology, 100, 379-390.

Loeber, R., \& Stouthamer-Loeber, M. (1998). Development of juvenile aggression and violence: Some common misconceptions and controversies. American Psychologist, 53, 242-259.

Loeber, R., Wung, P., Keenan, K., Giroux, B., Stouthamer-Loeber, M., Kammen, W. B. v., \& Maughan, B. (1993). Developmental pathways in disruptive child behavior.

Development and Psychopathology, 5, 101-132.

Loevinger, J. (1957). Objective tests as instruments of psychological theory. Psychological Reports, 3, 635-694.

MacCallum, R. C., Kim, C., Malarkey, W. B., \& Kiecolt-Glaser, J. K. (1997). Studying multivariate change using multilevel models and latent curve models. Multivariate Behavioral Research, 32, 215-253.

Mahoney, J. L., \& Bergman, L. R. (2002). Conceptual and methodological considerations in a developmental approach to the study of positive adaptation. Applied Developmental Psychology, 23, 195-217.

Masten, A. S., Coatsworth, J. D., Neemann, J., Gest, S. D., Tellegen, A., \& Garmezy, N. (1995). The structure and coherence of competence from childhood to adolescence. Child Development, 66, 1635-1659.

Matten, A. v. d. (1995). De Amsterdamse Schaal voor Opstandig gedrag. Unpublished Manuscript, Free University, Amsterdam.

Matthys, W., Maassen, G. H., Cuperus, J. M., \& van Engeland, H. (2001). The assessment of the situational specificity of children's problem behavior in peer-peer context. Journal of Child Psychology and Psychiatry and Allied Disciplines, 42, 413-420.

Maughan, B., Rower, R., Messer, J., Goodman, R., \& Meltzer, H. (2004). Conduct Disorder and Oppositional Defiant Disorder in a national sample: developmental epidemiology. Journal of Child Psychology and Psychiatry, 45, 609-621.

Meredith, W., \& Horn, J. (2001). The role of factorial invariance in modeling growth and change. In L. M. Collins \& A. G. Sayer (Eds.), New methods for the analysis of change (pp. 201-240). Washington, DC: American Psychological Association.

Mijné, L. (2003). Opstandigheid en de relaties van kinderen: De invloed van wederkerigheid, autoriteit, en emotionele betrokkenheid in de relatie met ouders, vrienden en de leerkracht [Oppositionality and children's relationships: The influence of reciprocity, 
authority, and closeness in relationships with parents, friends, and teachers]. Unpublished Doctoral Dissertation, Vrije Universiteit, Amsterdam.

Moffitt, T. E. (1993). Adolescent-limited and life-course-persistent antisocial behavior: A developmental taxonomy. Psychological Review, 100, 674-701.

Moffitt, T. E., Caspi, M., Rutter, M., \& Silva, P. A. (2001). Sex differences in antisocial behaviour: Conduct disorder, delinquency and violence in the Dunedin Longitudinal Study. New York: Cambridge University Press.

Moffitt, T. E., \& Silva, P. A. (1988). Self-reported delinquency: Results from an instrument for New Zealand. Australian and New Zealand Journal of Criminology, 21, 227-240.

Mones, A. G. (1998). Oppositional children and their families: An adaptational dance in space and time. American Journal of Orthopsychiatry, 68, 147-153.

Morgan, C. J., \& Cauce, A. M. (1999). Predicting DSM-III-R disorders from the youth selfreport: Analysis of data from a field study. Journal of the American Academy of Child \& Adolescent Psychiatry, 38, 1237-1245.

Munson, J. A., McMahon, R. J., \& Spieker, S. J. (2001). Structure and variability in the developmental trajectory of children's externalizing problems: Impact of attachment, maternal depressive symptomatology, and child sex. Development and Psychopathology, 13, 277-296.

Muris, P., Meesters, C., \& van den Berg, F. (2003). The Strengths and Difficulties Questionnaire: Further evidence for its reliability and validity in a community sample of Dutch children and adolescents. European Child and Adolescent Psychiatry, 12, 18.

Nagin, D. (1999). Analyzing developmental trajectories: A semi-parametric, group-based approach. Psychological Methods, 139-157.

Nagin, D., \& Tremblay, R. E. (1999). Trajectories of boys' physical aggression, opposition, and hyperactivity on the path to physically violent and nonviolent juvenile delinquency. Child Development, 70, 1181-1196.

Noom, M. J., Dekovic, M., \& Meeus, W. (2001). Conceptual analysis and measurement of adolescent autonomy. Journal of Youth and Adolescence, 30, 577-595.

Nottelmann, E. D., \& Jensen, P. S. (1995). Comorbidity of disorders in children and adolescents: Developmental perspectives. Advances in Clinical Child Psychology, 17, 109-155.

Offord, D. R., Boyle, M. H., Racine, Y., Szatmari, P., Fleming, J. E., Sanford, M., \& Lipman, E. L. (1996). Integrating assessment data from multiple informants. Journal of the American Academy of Child \& Adolescent Psychiatry, 35, 1078-1085.

Olweus, D. (1979). Stability of aggressive reaction patterns in males: A review. Psychological Bulletin, 852-875.

Osgood, D. W., McMorris, B. J., \& Potenza, M. T. (2002). Analyzing multiple-item measures of crime and deviance I: Item response theory scaling. Journal of Quantitative Criminology, 18, 267-295.

Paikoff, R. L., \& Brooks-Gunn, J. (1991). Do parent-child relationships change during puberty? Psychological Bulletin, 110, 47-66. 
Parker, J. G., \& Gottman, J. M. (1989). Social and emotional development in a relational context. In J. Berndt \& G. W. Ladd (Eds.), Peer relationships in child development (pp. 95-131). New York: Wiley.

Patterson, G. R., DeBaryshe, B. D., \& Ramsey, E. (1989). A developmental perspective on antisocial behavior. American Psychologist, 44, 329-355.

Phares, V., Compas, B. E., \& Howell, D. C. (1989). Perspectives on child behavior problems: Comparisons of children's self-reports with parent and teacher reports. Psychological Assessment, 1, 68-71.

Plewis, I. (2001). Explanatory models for relating growth processes. Multivariate Behavioral Research, 36, 207-225.

Rasbash, J., Browne, W., Goldstein, H., Yang, M., Plewis, I., Healy, M., Woodhouse, G., Draper, D., Langford, I., \& Lewis, T. (2000). A user's guide to MLwiN, Version 2.1. London: University of London, Institute of Education.

Redl, F. (1976). Oppositional behavior in everyday life. In A. J. Anthony \& D. C. Gilpin (Eds.), Three clinical faces of childhood (pp. 17-28). New York: Spectrum.

Reis, H. T., Collins, A. W., \& Berscheid, E. (2000). The relationship context of human behavior and development. Psychological Bulletin, 126, 844-872.

Rey, J. M., Bashir, M. R., Schwarz, M., Richards, I. N., Plapp, J. M., \& Stewart, G. W. (1988). Oppositional disorder: Fact or fiction? Journal of the American Academy of Child and Adolescent Psychiatry, 27, 157-162.

Rey, J. M., \& Walter, G. (1999). Oppositional Defiant Disorder. In R. L. Hendren (Ed.), Disruptive behavior disorders in children and adolescents (Vol. 18, pp. 99-126). Washington DC: American Psychiatric Press.

Rowe, D. C., \& Kandel, D. (1997). In the eye of the beholder? Parental ratings of externalizing and internalizing symptoms. Journal of Abnormal Child Psychology, 25, 265-275.

Rowe, R., Maughan, B., Pickles, A., Costello, J. E., \& Angold, A. (2002). The relationship between DSM-IV oppositional defiant disorder and conduct disorder: findings from the Great Smoky Mountains Study. Journal of Child Psychology and Psychiatry, 43, 365-373.

Ruchkin, V., \& Schwab-Stone, M. (2003). What can we learn from developmental studies of psychiatric disorders? The Lancet, 362, 1951-1952.

Rudinger, G., \& Reitz, C. (1998). The neglected time dimension?: Introducing a longitudinal model testing latent growth curves, stability, and reliability as time bound processes. Methods of Psychological Research, 3, 1-15 (http://www.mpr-online.de).

Rudolph, K. D. (2002). Gender differences in emotional responses to interpersonal stress during adolescence. Journal of Adolescent Health, 30, 3-13.

Ruscio, J., \& Ruscio, A. M. (2004). Claryfying boundary issues in psychpathology: The role of taxometrics in a comprehensive program of structural research. Journal of Abnormal Psychology, 113, 24-38. 
Rutter, M., Caspi, A., \& Moffitt, T. E. (2003). Using sex differences in psychopathology to study causal mechanisms: Unifying issues and research strategies. Journal of Child Psychology and Psychiatry, 44, 1092--1115.

Schaeffer, C. M., Petras, H., Ialongo, N., Poduska, J., \& Kellam, S. (2003). Modeling growth in boys' aggressive behavior across elementary school: Links to later criminal involvement, conduct disorder and antisocial personality disorder. Developmental Psychology, 39, 1020-1035.

Shaw, B. A., Krause, N., Chatters, L. M., Connell, C. M., \& Ingersoll-Dayton, B. (2004). Emotional support from parents early in life, aging, and health. Psychology and Aging, 19, 4-12.

Silverthorn, P., \& Frick, P. J. (1999). Developmental pathways to antisocial behavior: The delayed-onset pathway in girls. Development and Psychopathology, 11, 101-126.

Smetana, J. G. (2000). Middle-class African American adolescents' and parents' conceptions of parental authority and parenting practices: A longitudinal investigation. Child Development, 71, 1672-1686.

Smith, B. H., Pelham, W. E., Gnagy, E., Molina, B., \& Evans, S. (2000). The reliability, validity, and unique contributions of self-report by adolescents receiving treatment for Attention/Deficit/Hyperactivity Disorder. Journal of Consulting and Clinical Psychology, 68, 489-499.

Snijders, T., \& Bosker, R. (1999). Multilevel analysis: An introduction to basic and advanced multilevel modeling. London etc.: Sage Publishers.

Sroufe, L. A. (1997). Psychopathology as an outcome of development. Development and Psychopathology, 9, 251-268.

Steiger, J. H. (1980). Tests for comparing elements of a correlation matrix. Psychological Bulletin, 87, 245-251.

Steinberg, L. (2000). The family at adolescence: Transition and transformation. Journal of Adolescent Health, 27, 170-178.

Stice, E., Ragan, J., \& Randall, P. (2004). Prospective relations between social support and depression: Differential direction of effects for parent and peer support? Journal of Abnormal Psychology, 113, 155-159.

Stifter, C. A., Spinrad, T. L., \& Braungart-Rieker, J. M. (1999). Toward a developmental model of child compliance: The role of emotion regulation in infancy. Child Development, 70, 21-32.

Stormshak, E. A., \& Webster-Stratton, C. (1999). The qualitative interactions of children with conduct problems and their peers: Differential correlates with self-report measures, home behavior, and school behavior problems. Journal of Applied Developmental Psychology, 20, 295-317.

Tanaka, J. S., \& Huba, G. J. (1985). A fit index for covariance structure models under arbitrary GLS estimation. British Journal of Mathematical and Statistical Psychology, $42,233-239$. 
Tremblay, R. E., Loeber, R., Gagnon, C., Charlebois, P., Larivee, S., \& Leblanc, M. (1991). Disruptive boys with stable and unstable high fighting behavior patterns during junior elementary school. Journal of Abnormal Child Psychology, 19, 285-300.

Underwood, M. K., Shockner, A. E., \& Hurley, J. C. (2001). Children's responses to sameand other-gender peers: An experimental investigation with 8-, 10- and 12-year-olds. Developmental Psychology, 37, 362-372.

van der Ende, J. (1999). Multiple informants: Multiple views. In H. M. Koot, A. A. M. Crijnen, \& R. F. Ferdinand (Eds.), Child psychiatric epidemiology. Accomplishments and future directions (pp. 39-52). Assen, The Netherlands: Van Gorcum.

Vermeiren, R. (2003). Psychopathology and delinquency in adolescents: A descriptive and developmental perspective. Clinical Psychology Review, 23, 277-318.

Wakefield, J. C. (1993). The limits of operationalization: A critique of Spitzer and Endicott's (1978) proposed operational criteria for mental disorder. Journal of Abnormal Psychology, 102, 160-172.

Wångby, M., Bergman, L. R., \& Magnusson, D. (1999). Development of adjustment problems in girls: What syndromes emerge? Child Development, 70, 678-699.

Webster-Stratton, C. (1990). Long-term follow-up of families with young conduct problem children: From preschool to grade school. Journal of Clinical Child Psychology, 19, 144-149.

Wenar, C. (1982). On negativism. Human Development, 25, 1-23.

Wenning, K., Nathan, P., \& King, S. (1993). Mood disorders in children with oppositional defiant disorder: A pilot study. American Journal of Orthopsychiatry, 63, 295-299.

Widenfelt, B. M., Goedhart, A. W., Treffers, P. D. A., \& Goodman, R. (2003). Dutch version of the Strengths and Difficulties Questionnaire (SDQ). European Child \& Adolescent Psychiatry, 12, 281-289.

Widiger, T. A., \& Clark, L. A. (2000). Toward DSM-V and the classification of psychopathology. Psychological Bulletin, 126, 946-963.

Willoughby, M., Kupersmidt, J., \& Bryant, D. (2001). Overt and covert dimensions of antisocial behavior in early childhood. Journal of Abnormal Child Psychology, 29, 177-187.

Willoughby, M. T. (2003). Developmental course of ADHD symptomatology during the transition from childhood to adolescence: A review with recommendations. Journal of Child Psychology and Psychiatry, 44, 88-106.

Wohlwill, J. F. (1973). The study of behavioral development. New York: Academic Press.

Wood, J. M., Garb, H. N., Lilienfeld, S. O., \& Nezworski, M. T. (2002). Clinical assessment. Annual Review of Psychology, 53, 519-543.

Wright, J. C., \& Zakriski, A. L. (2001). A contextual analysis of externalizing and mixed syndrome boys: When syndromal similarity obscures functional dissimilarity. Journal of Consulting and Clinical Psychology, 69, 457-470.

Wright, J. C., \& Zakriski, A. L. (2003). When syndromal similarity obscures functional dissimilarity: Distinctive evoked environments of externalizing and mixed syndrome boys. Journal of Consulting and Clinical Psychology, 71, 516-527. 
References

Wright, J. C., Zakriski, A. L., \& Drinkwater, M. (1999). Developmental psychopathology and the reciprocal patterning of behavior and environment: Distinctive situational and behavioral signatures of internalizing, externalizing and mixed-syndrome children. Journal of Consulting and Clinical Psychology, 67, 95-107.

Youngstrom, E., Loeber, R., \& Stouthamer-Loeber, M. (2000). Patterns and correlates of agreement between parent, teacher, and male adolescent ratings of externalizing and internalizing problems. Journal of Consulting and Clinical Psychology, 68, 10381050.

Youniss, J. (1980). Parents and peers in social development. Chicago: University of Chicago Press. 